\title{
Dimensional reduction and the long-time behavior of Ricci flow
}

John Lott*

\begin{abstract}
If $g(t)$ is a three-dimensional Ricci flow solution, with sectional curvatures that are $O\left(t^{-1}\right)$ and diameter that is $O\left(t^{1 / 2}\right)$, then the pullback Ricci flow solution on the universal cover approaches a homogeneous expanding soliton.
\end{abstract}

Mathematics Subject Classification (2010). 53C44, 57M50.

Keywords. Ricci flow, geometrization.

\section{Introduction}

After Perelman's proof of Thurston's geometrization conjecture [43], [44], using Hamilton's Ricci flow [24], there are many remaining questions about three-dimensional Ricci flow.

Since the Ricci flow is a nonlinear heat equation for the Riemannian metric, the intuition is that it should smooth out the metric and thereby give rise, in the longtime limit, to the locally homogeneous pieces in the geometric decomposition. This intuition is a bit misleading because, for example, of the presence of singularities in the Ricci flow. Nevertheless, based partly on earlier work of Hamilton [27], Perelman showed that the hyperbolic pieces do asymptotically appear in the Ricci flow. Perelman's proof for the existence of the other geometric pieces is more indirect. Perelman showed that the nonhyperbolic part of the evolving manifold satisfies certain geometric conditions, from which one can show that it is a graph manifold [1], [32], [42], [44], [51]. By earlier work of topologists, graph manifolds have a geometric decomposition.

It is an open question whether the Ricci flow directly performs the geometric decomposition of a three-manifold, as time evolves. In particular, suppose that the geometric decomposition of the three-manifold consists of a single geometric piece. If this piece has Thurston type $S^{3}$ or $S^{1} \times S^{2}$ then its Ricci flow has a finite extinction time [10], [11], [45]. For the other Thurston types, one can ask whether the large-time behavior of the Ricci flow solution will be that of a locally homogeneous Ricci flow, no

\footnotetext{
*This work was supported by NSF grant DMS-0604829.
} 
matter what the initial metric may be. Hamilton [25, Section 11], Hamilton-Isenberg [28] and Knopf [33] showed that this is true for certain manifolds of $\mathbb{R}^{3}$ or Soltype if one assumes some extra symmetries on the initial metric. We are interested in whether one can show asymptotic homogeneity for a wider class of Ricci flow solutions.

To describe the results, let $g(\cdot)$ denote a Ricci-flow-with-surgery whose initial manifold is a closed orientable 3-manifold. Let $M_{t}$ denote the time- $t$ manifold. (If $t$ is a surgery time then we take $M_{t}$ to be the postsurgery manifold.) From Perelman's work [45], there is some time $T_{0}$ so that for all $t \geq T_{0}$, each connected component $C$ of $M_{t}$ is $S^{3}$ or an aspherical 3-manifold. As the geometrization conjecture holds, $C$ has a decomposition into geometric pieces of type $S^{3}, \mathbb{R}^{3}, H^{3}$, Nil, Sol, $H^{2} \times \mathbb{R}$ and $\widetilde{S L_{2}(\mathbb{R})}$; see Section 2 .

It is possible that the Ricci-flow-with-surgery involves an infinite number of surgeries. In the known examples, there is a finite number of surgeries. Furthermore, in the known examples, after all of the surgeries are done then the sectional curvatures uniformly decay in magnitude as $O\left(t^{-1}\right)$, i.e., one has a type-III Ricci flow solution. In order to make progress, we will consider only Ricci-flows-with-surgery in which this is the case. Hence, we will consider a smooth Ricci flow $(M, g(\cdot))$, defined for $t \in(1, \infty)$ on a closed, connected orientable 3-manifold $M$, with sectional curvatures that are uniformly $O\left(t^{-1}\right)$.

If $M$ admits a locally homogeneous metric modeled on a given one of the eight Thurston geometries then we will say that $M$ has the corresponding Thurston type. Saying that $M$ has a certain Thurston type is a topological statement, i.e., we allow ourselves to consider Riemannian metrics on $M$ that are not locally homogeneous.

In order to analyze the large-time behavior of a Ricci flow, we use blowdown limits.

Definition 1.1. For $s \geq 1$, put $g_{s}(t)=\frac{1}{s} g(s t)$. It is also a Ricci flow solution. Let $\tilde{g}_{s}(t)$ be the lift of $g_{s}(t)$ to the universal cover $\tilde{M}$.

A time interval $[a, b]$ for $g_{s}$ corresponds to the time interval $[s a, s b]$ for $g$. We are interested in the behavior as $s \rightarrow \infty$ of $g_{s}(\cdot)$ on a specified time interval $[a, b]$, since this gives information about the large-time behavior of the initial Ricci flow solution $g(\cdot)$. If there is a limiting Ricci flow solution $\lim _{s \rightarrow \infty} g_{s}(\cdot)$ then one says that it is a blowdown limit of $g(\cdot)$.

For notation, if the Gromov-Hausdorff $\operatorname{limit}_{t \rightarrow \infty}\left(M, \frac{g(t)}{t}\right)$ exists and equals a compact metric space $X$ then we write $\lim _{t \rightarrow \infty}\left(M, \frac{g(t)}{t}\right) \stackrel{\text { GH }}{=} X$. If we write $\lim _{s \rightarrow \infty}\left(\tilde{M}, \tilde{m}, \tilde{g}_{s}(\cdot)\right)=\left(M_{\infty}, m_{\infty}, g_{\infty}(\cdot)\right)$ then we mean that for any sequence $\left\{s_{j}\right\}_{j=1}^{\infty}$ tending to infinity, there is a smooth pointed $\operatorname{limit}_{j \rightarrow \infty}\left(\tilde{M}, \tilde{m}, \tilde{g}_{s_{j}}(\cdot)\right)$ of Ricci flow solutions which equals $\left(M_{\infty}, m_{\infty}, g_{\infty}(\cdot)\right)$. We recall that the notion 
of the limit in the statement $\lim _{j \rightarrow \infty}\left(\tilde{M}, \tilde{m}, \tilde{g}_{s_{j}}(\cdot)\right)=\left(M_{\infty}, m_{\infty}, g_{\infty}(\cdot)\right)$ involves $j$-dependent pointed diffeomorphisms from domains in $M_{\infty}$ to domains in $\tilde{M}$ [26].

Theorem 1.2. Let $(M, g(\cdot))$ be a smooth Ricci flow solution on a connected closed orientable 3-manifold, defined for $t \in(1, \infty)$. Suppose that the sectional curvatures of $(M, g(t))$ are uniformly $O\left(t^{-1}\right)$ and $\operatorname{diam}(M, g(t))=O\left(t^{\frac{1}{2}}\right)$.

Then $M$ is irreducible, aspherical and its geometric decomposition contains a single geometric piece.

1. If $M$ has Thurston type $\mathbb{R}^{3}$ then $\lim _{t \rightarrow \infty}\left(M, \frac{g(t)}{t}\right) \stackrel{\mathrm{GH}}{=} \mathrm{pt}$.

The limit $\lim _{s \rightarrow \infty}\left(\tilde{M}, \tilde{m}, \tilde{g}_{s}(\cdot)\right)$ exists and equals the flat expanding soliton

$$
\left(\mathbb{R}^{3}, g_{\text {flat }}\right) \text {. }
$$

2. If $M$ has Thurston type Nil then $\lim _{t \rightarrow \infty}\left(M, \frac{g(t)}{t}\right) \stackrel{\mathrm{GH}}{=} \mathrm{pt}$.

The limit $\lim _{s \rightarrow \infty}\left(\tilde{M}, \tilde{m}, \tilde{g}_{s}(\cdot)\right)$ exists and equals the expanding soliton

$$
\left(\mathbb{R}^{3}, \frac{1}{3 t^{\frac{1}{3}}}\left(d x+\frac{1}{2} y d z-\frac{1}{2} z d y\right)^{2}+t^{\frac{1}{3}}\left(d y^{2}+d z^{2}\right)\right) .
$$

3. If $M$ has Thurston type Sol then the Gromov-Hausdorff limit $\lim _{t \rightarrow \infty}\left(M, \frac{g(t)}{t}\right)$ is a circle or an interval.

The limit $\lim _{s \rightarrow \infty}\left(\tilde{M}, \tilde{m}, \tilde{g}_{s}(\cdot)\right)$ exists and equals the expanding soliton

$$
\left(\mathbb{R}^{3}, e^{-2 z} d x^{2}+e^{2 z} d y^{2}+4 t d z^{2}\right) .
$$

4. If $M$ has Thurston type $H^{2} \times \mathbb{R}$ then for any sequence $\left\{t_{j}\right\}_{j=1}^{\infty}$ tending to infinity, there is a subsequence (which we relabel as $\left\{t_{j}\right\}_{j=1}^{\infty}$ ) so that the GromovHausdorff limit $\lim _{j \rightarrow \infty}\left(M, \frac{g\left(t_{j}\right)}{t_{j}}\right)$ exists and is a metric of constant curvature $-\frac{1}{2}$ on a closed 2-dimensional orbifold.

The limit $\lim _{s \rightarrow \infty}\left(\tilde{M}, \tilde{m}, \tilde{g}_{s}(\cdot)\right)$ exists and equals the expanding soliton

$$
\left(H^{2} \times \mathbb{R}, 2 t g_{\text {hyp }}+g_{\mathbb{R}}\right) .
$$

5. If $M$ has Thurston type $H^{3}$ then $\lim _{t \rightarrow \infty}\left(M, \frac{g(t)}{t}\right) \stackrel{\mathrm{GH}}{=}\left(M, 4 g_{\text {hyp }}\right)$.

The limit $\lim _{s \rightarrow \infty}\left(\tilde{M}, \tilde{m}, \tilde{g}_{s}(\cdot)\right)$ exists and equals the expanding soliton

$$
\left(H^{3}, 4 \operatorname{tg}_{\text {hyp }}\right) \text {. }
$$

6. If $M$ has Thurston type $\widetilde{\mathrm{SL}_{2}(\mathbb{R})}$ then there is some sequence $\left\{s_{j}\right\}_{j=1}^{\infty}$ tending to infinity such that $\lim _{j \rightarrow \infty}\left(M, \frac{g\left(s_{j}\right)}{s_{j}}\right)$ is a metric of constant curvature $-\frac{1}{2}$ on a closed 2-dimensional orbifold.

The limit $\lim _{j \rightarrow \infty}\left(\tilde{M}, \tilde{m}, \tilde{g}_{s_{j}}(\cdot)\right)$ is the expanding soliton

$$
\left(H^{2} \times \mathbb{R}, 2 t g_{\text {hyp }}+g_{\mathbb{R}}\right) .
$$


Corollary 1.3. In cases 1-5 of Theorem 1.2, as time becomes large the Ricci flow solution becomes increasingly locally homogeneous.

The corollary follows from the fact that the expanding solitons in Theorem 1.2 are all homogeneous. To state the corollary in a more precise way, we recall that a Riemannian manifold $(M, g)$ is locally homogeneous if and only if any function on $M$ that can be expressed as a polynomial in the covariant derivatives of the curvature tensor $\nabla_{i_{1}} \nabla_{i_{2}} \ldots \nabla_{i_{r}} R_{j k l m}$ and the inverse metric tensor $g^{i j}$, by contracting indices, is actually constant on $M$ [47]. Corollary 1.3 means that in cases $1-5$ of Theorem 1.2, any function on $M$ which is a polynomial in the covariant derivatives of the curvature tensor and the inverse metric tensor of the rescaled metric $\hat{g}(t)=\frac{g(t)}{t}$ approaches a constant value as $t \rightarrow \infty$.

Remark 1.4. The diameter condition $\operatorname{diam}(M, g(t))=O\left(t^{\frac{1}{2}}\right)$ implies (under our curvature assumption) that the geometric decomposition of $M$ contains a single geometric piece; see Proposition 3.5. We expect that if the diameter condition is not satisfied then the geometric decomposition of $M$ will contain more than one geometric piece.

Remark 1.5. Any locally homogeneous Ricci flow solution $(M, g(\cdot))$ on a closed 3-manifold $M$, which exists for $t \in(1, \infty)$, does have sectional curvatures that are uniformly $O\left(t^{-1}\right)$ and $\operatorname{diam}(M, g(t))=O\left(t^{\frac{1}{2}}\right)$ [29], [35]. Hence a Ricci flow solution on $M$ that in any reasonable sense approaches a locally homogeneous solution, as time goes to infinity, will satisfy the assumptions of Theorem 1.2. In this way, Theorem 1.2 is essentially an if and only if statement.

Remark 1.6. In Case 6 of Theorem 1.2 we only show that we have the desired limit for some sequence $\left\{s_{j}\right\}_{j=1}^{\infty}$ tending to infinity, not for any such sequence. The reason is a technical point about local stability; see Remark 6.5.

In [38, Theorem 1.1] we showed that the expanding soliton solutions listed in Theorem 1.2 are universal attractors within the space of homogeneous Ricci flow solutions on Thurston geometries. In proving Theorem 1.2, we show that they are global attractors within the space of Ricci flow solutions that satisfy the given curvature and diameter assumptions, after passing to the universal cover.

Theorem 1.2 describes the Gromov-Hausdorff limit of the rescaled Ricci flow solution on $M$ and the smooth pointed rescaling limit of the lifted Ricci flow solution on $\tilde{M}$. In the proof we show there is a rescaling limit which is a Ricci flow solution on an object that simultaneously encodes both the Gromov-Hausdorff limit on $M$ and the smooth limit on $\tilde{M}$. This rescaling limit can be considered to give a canonical geometry for $M$. A similar phenomenon occurs in the work of Song and Tian concerning collapsing in the Kähler-Ricci flow on elliptic fibrations [52]. 
There are three main tools in the proof of Theorem 1.2: a compactness theorem, a monotonicity formula and a local stability result. The compactness theorem [38, Theorem 5.12] is an extension of Hamilton's compactness theorem for Ricci flow solutions [26]. Hamilton's theorem allows one to take a convergent subsequence of a sequence of pointed Ricci flow solutions that have uniform curvature bounds on compact time intervals and a uniform lower bound on the injectivity radius at the basepoint. The rescalings of a Ricci flow solution on a manifold $M$, as considered in Theorem 1.2, may collapse, i.e., the Gromov-Hausdorff limit $X$ may have dimension less than three. This means that there is no uniform lower bound on the injectivity radius of the rescaled solution, and so there cannot be a limiting Ricci flow solution on a 3-manifold. Instead, the limiting Ricci flow solution lives on a more general object called an étale groupoid. Roughly speaking, an étale groupoid combines the notions of manifold and discrete group into a single object. Its relevance for us comes from the Cheeger-Fukaya-Gromov theory of bounded curvature collapse [3], which implies that a Riemannian manifold which collapses with bounded sectional curvature will asymptotically acquire extra symmetries. In Section 3 we give a brief overview of how collapsing interacts with Ricci flow.

Under the assumptions of Theorem 1.2, the compactness theorem of [38] implies that if $\left\{s_{j}\right\}_{j=1}^{\infty}$ is a sequence tending to infinity then after passing to a subsequence, $\left\{\left(M, g_{s_{j}}(\cdot)\right)\right\}_{j=1}^{\infty}$ converges to a Ricci flow solution $\bar{g}(\cdot)$ on a three-dimensional étale groupoid. It remains to understand the long-time behavior of $\bar{g}(\cdot)$. In our case, the relevant étale groupoids arise from locally free abelian group actions. In essence, we have to understand the long-time behavior of an invariant Ricci flow solution on the total space of a (twisted) abelian principal bundle over a compact space $B$. Such a Ricci flow solution $\bar{g}(\cdot)$ becomes a coupled system of evolution equations on the lower-dimensional space $B$. This is the dimensional reduction part of the title of this paper.

Our main tool to analyze the long-time behavior of such a Ricci flow is a modification of the Feldman-Ilmanen-Ni expanding entropy functional $\mathcal{W}_{+}[14]$, which in turn is a variation on Perelman's W-functional [43]. More generally, in Section 4 we describe versions of the $\mathcal{F}, \mathcal{W}$ and $\mathcal{W}_{+}$functionals that are adapted for abelian actions. Using the modified $\mathcal{W}_{+}$functional, we show that any blowdown limit of $\bar{g}(\cdot)$ satisfies the harmonic-Einstein equations of [38]. As we are in dimension three, we can solve the harmonic-Einstein equations to find the homogeneous expanding soliton solutions of Theorem 1.2.

By these techniques, we show that there is some sequence $\left\{s_{j}\right\}_{j=1}^{\infty}$ tending to infinity so that $\left\{\left(M, g_{s_{j}}(\cdot)\right)\right\}_{j=1}^{\infty}$ converges in an appropriate sense to a locally homogeneous expanding soliton solution. In order to get convergence for all sequences $\left\{s_{j}\right\}_{j=1}^{\infty}$ tending to infinity, we use the local stability of the locally homogeneous expanding solitons, along with some further arguments. The local stability is due to Dan Knopf [34]. An important point is that we only need the local stability of the 
locally homogeneous expanding soliton within the space of Ricci flow solutions with the same abelian symmetry. Because of this, the local stability issue reduces to an elliptic-type analysis on the compact quotient space $B$ where one has compact resolvents, etc. For the Nil and Sol-expanders, the local stability in a somewhat different sense was considered in [21].

The outline of this paper is as follows. In Section 2 we make some general remarks about Ricci flow and geometrization. In Section 3 we give an overview of some of the needed results from [38]. In Section 4, which may be of independent interest, we analyze Ricci flow solutions with a locally free abelian group action. In Section 5 we give the classification of the étale groupoids that arise. In Section 6 we prove Theorem 1.2. Further descriptions are given at the beginnings of the sections.

I thank Xiaodong Cao, Dan Knopf and Junfang Li for discussions on the topics of this paper. I am especially grateful to Dan for telling me of his local stability results [34]. Part of this research was performed while attending the MSRI 2006-2007 program on Geometric Evolution Equations. I thank MSRI and the UC-Berkeley Mathematics Department for their hospitality, along with the organizers of the MSRI program for inviting me.

\section{Geometrization conjecture and Ricci flow}

In this section we describe what one might expect for the long-time behavior of the Ricci flow on a compact 3-manifold $M$, in terms of the geometric decomposition of $M$. Background information on the geometrization conjecture is in [50].

Let $M$ be a connected closed orientable 3-manifold. The Kneser-Milnor theorem says that $M$ has a connected sum decomposition $M=M_{1} \# M_{2} \# \ldots \# M_{N}$ into socalled prime factors, unique up to permutation. Thurston's geometrization conjecture says that if $M$ is prime then there is a (possibly empty) minimal collection of disjoint incompressible embedded 2-tori $\left\{T_{i}\right\}_{i=1}^{I}$ in $M$, unique up to isotopy, so that each connected component of $M-\bigcup_{i=1}^{I} T_{i}$ admits a complete locally homogeneous metric of one of the following types:

1. A compact quotient of $S^{3}, S^{2} \times \mathbb{R}, \mathbb{R}^{3}$, Nil, Sol, $H^{3}, H^{2} \times \mathbb{R}$ or $\widetilde{\mathrm{SL}_{2}(\mathbb{R})}$.

2. A noncompact finite-volume quotient of $H^{3}$ or $H^{2} \times \mathbb{R}$.

3. $\mathbb{R} \times_{\mathbb{Z}_{2}} T^{2}$, where the generator of $\mathbb{Z}_{2}$ acts by $x \rightarrow-x$ on $\mathbb{R}$ and by the involution on $T^{2}$ for which $T^{2} / \mathbb{Z}_{2}$ is the Klein bottle $K$.

Remark 2.1. A finite-volume quotient of $S^{3}, S^{2} \times \mathbb{R}, \mathbb{R}^{3}$, Nil or Sol is necessarily a compact quotient. Noncompact finite-volume quotients of $\widetilde{\mathrm{SL}_{2}(\mathbb{R})}$ are not on the list, as they are diffeomorphic to noncompact finite-volume quotients of $H^{2} \times \mathbb{R}$. 
Remark 2.2. If we were to cut along both 2-tori and Klein bottles then we could eliminate the $\mathbb{R} \times_{\mathbb{Z}_{2}} T^{2}$ case, which is the total space of a twisted $\mathbb{R}$-bundle over $K$. However, as we are dealing with orientable manifolds, it is more natural to only cut along 2-tori.

We now discuss graph manifolds. A reference is Chapter 2.4 in [39]. We recall that a compact orientable 3-manifold $M$ with (possibly empty) boundary is a graph manifold if there is a collection of disjoint embedded 2-tori $\left\{T_{j}\right\}_{j=1}^{J}$ so that if we take the metric completion of $M-\bigcup_{j=1}^{J} T_{j}$ (with respect to some Riemannian metric on $M$ ) then each connected component is the total space of a circle bundle over a compact surface. Clearly $\partial M$, if nonempty, is a disjoint union of 2-tori. The result of gluing two graph manifolds along boundary components is again a graph manifold (provided that it is orientable). In addition, the connected sum of two graph manifolds is a graph manifold. In terms of the Thurston decomposition, a closed orientable prime 3-manifold $M$ is a graph manifold if and only if it has no hyperbolic pieces.

We now summarize how Perelman proved the geometrization conjecture using Ricci flow. If $g(0)$ is an initial Riemannian metric on $M$ then Perelman showed that there is a Ricci-flow-with-surgery $\left(M_{t}, g(t)\right)$ defined for all $t \in[0, \infty)$ (although $M_{t}$ may become the empty set for large $t$ ). A singularity in the flow is handled by letting some connected components go extinct or by performing surgery. If $t$ is a surgery time then we let $M_{t}$ denote the postsurgery manifold $M_{t}^{+}$. Going from a postsurgery manifold $M_{t}^{+}$to the presurgery manifold $M_{t}^{-}$amounts topologically to performing connected sums on some components of $M_{t}^{+}$, possibly along with a finite number of $S^{1} \times S^{2}$ 's and $\mathbb{R} P^{3}$ 's, and restoring any factors that went extinct at time $t$. From Kneser's theorem, there is some $T_{1}>0$ so that for a singularity time $t>T_{1}, M_{t}^{+}$ differs from $M_{t}^{-}$by the addition or subtraction of some $S^{3}$ factors. That is, after time $T_{1}$, all surgeries are topologically trivial.

Perelman showed that any connected component which goes extinct during the Ricci-flow-with-surgery is diffeomorphic to $S^{1} \times S^{2}, S^{1} \times_{\mathbb{Z}_{2}} S^{2}=\mathbb{R} P^{3} \# \mathbb{R} P^{3}$ or $S^{3} / \Gamma$, where $\Gamma$ is a finite subgroup of $\mathrm{SO}(4)$ that acts freely on $S^{3}$. He also showed that for large $t$, any connected component $C$ of $M_{t}$ has a 3-dimensional submanifold $G$ with (possibly empty) boundary so that $G$ is a graph manifold, $\partial G$ consists of incompressible tori in $C$ and $C-G$ admits a complete finite-volume hyperbolic metric. Here $G$ is allowed to be $\varnothing$ or $C$. Using earlier results from 3-manifold topology, this is enough to prove the geometrization conjecture.

It is not known whether there is a finite number of surgeries, but after some time all remaining surgeries will occur in the graph manifold part. For example, if the original manifold $M$ admits a hyperbolic metric then there is a finite number of surgeries, since for large time there is no graph manifold part. We note that one can never exclude singularities for topological reasons, as the initial metric could always contain a pinched 2-sphere. 
In [45], Perelman showed that for large $t$, any connected component of $M_{t}$ is aspherical or $S^{3}$. Thus the relevant Thurston geometries are $S^{3}, \mathbb{R}^{3}, \mathrm{Nil}, \mathrm{Sol}, H^{3}$, $H^{2} \times \mathbb{R}$ and $\widetilde{\mathrm{SL}_{2}(\mathbb{R})}$.

Put $\hat{g}(t)=\frac{g(t)}{t}$. Let us assume that there is a finite number of surgeries, and consider the manifold $M$ to be a connected component of the remaining manifold after all of the surgeries are performed. Based on explicit calculations for the Ricci flow on a locally homogeneous 3-manifold, the most optimistic possibility for the Gromov-Hausdorff behavior of the long-time Ricci flow is given in the following table. Here $X$ is the Gromov-Hausdorff $\operatorname{limit}_{t \rightarrow \infty}(M, \hat{g}(t))$, which we assume to exist. The "Thurston type" denotes the possible geometric types in the Thurston decomposition of $M$, but we do not assume that the metrics in the Ricci flow are locally homogeneous.

\begin{tabular}{c|c}
$X$ & Thurston type \\
\hline $\mathrm{pt}$. & $\mathbb{R}^{3}$ or Nil \\
$S^{1}$ or $I$ & Sol \\
closed 2-orbifold with $K=-1 / 2$ & $H^{2} \times \mathbb{R}$ or $\widetilde{\mathrm{SL}_{2}(\mathbb{R})}$ \\
closed 3-manifold with $K=-1 / 4$ & $H^{3}$ \\
noncompact & $H^{3}, H^{2} \times \mathbb{R}, \mathbb{R}^{3}$
\end{tabular}

If $X$ is noncompact then the possible geometric pieces in the geometric decomposition of $M$ should be noncompact finite-volume quotients of $H^{3}$, noncompact finite-volume quotients of $H^{2} \times \mathbb{R}$ and copies of $\mathbb{R} \times \mathbb{Z}_{2} T^{2}$. (The final $\mathbb{R}^{3}$-term in the table refers to the latter possibility.) When discussing Gromov-Hausdorff limits in this case, one would have to choose a basepoint $m \in M$ and take a pointed GromovHausdorff limit $(X, x) \stackrel{\mathrm{GH}}{=} \lim _{t \rightarrow \infty}(M, m, \hat{g}(t))$, whose value would depend on $m$. One would expect to get possible Gromov-Hausdorff limits of the form

1. $H^{3} / \Gamma$, where $\Gamma$ is a torsion-free noncocompact lattice in $\operatorname{PSL}(2, \mathbb{C})$;

2. $H^{2} / \Gamma$, where $\Gamma$ is a noncocompact lattice in $\operatorname{PSL}(2, \mathbb{R})$;

3. $\mathbb{R}$;

4. $[0, \infty)$.

Example 2.3. Suppose that $M=N \cup_{T_{2}} \bar{N}$ is the double of the truncation $N$ of a singly-cusped finite-volume hyperbolic 3-manifold $Y$, where the metric on $N$ is perturbed to make it a product near $\partial N$. If $m$ is in $N-T^{2}$ then one would expect that $\lim _{t \rightarrow \infty}(M, m, \hat{g}(t)) \stackrel{\mathrm{GH}}{=} Y$, with a metric of constant curvature $-\frac{1}{4}$, while if $m \in T^{2}$ then one would expect that $\lim _{t \rightarrow \infty}(M, m, \hat{g}(t)) \stackrel{\mathrm{GH}}{=} \mathbb{R}$. 
Example 2.4. Put $M^{\prime}=N \cup_{T^{2}}\left(I \times_{Z_{2}} T^{2}\right)$, where $I \times_{\mathbb{Z}_{2}} T^{2}$ is the (orientable) total space of a twisted interval bundle over the Klein bottle $K$. Then $M^{\prime}$ is double covered by $N \cup_{T^{2}} N$, where the gluing is done by an orientation-reversing isometry of $T^{2}$. If $m \in M^{\prime}-K$ then one would expect that $\lim _{t \rightarrow \infty}\left(M^{\prime}, m, \hat{g}(t)\right) \stackrel{\mathrm{GH}}{=} Y$, while if $m \in K$ then one would expect that $\lim _{t \rightarrow \infty}\left(M^{\prime}, m, \hat{g}(t)\right) \stackrel{\mathrm{GH}}{=} \mathbb{R} / \mathbb{Z}_{2}=[0, \infty)$.

This example shows why, from the point of view of Ricci flow, it is natural to include $\mathbb{R} \times \mathbb{Z}_{2} T^{2}$ as part of the geometric decomposition; see Remark 2.2. (In this sense it would also be natural to include $\mathbb{R} \times T^{2}$ as a possible piece, but such a piece would be topologically redundant.)

In the collapsing case, i.e., when $\operatorname{dim}(X)<3$, the Gromov-Hausdorff limit $X$ contains limited information about the evolution of the 3-dimensional geometry under the Ricci flow. For $t$ large, any component of the time- $t$ manifold is aspherical or $S^{3}$. Because of this, one natural way to get more information about the 3-dimensional geometry is to look at the evolving geometry on the universal cover. A special case is when $M$ is locally homogeneous. In [38, Section 3] the Ricci flow was considered on a simply-connected homogeneous 3-manifold $G / H$, where $G$ is a connected unimodular Lie group and $H$ is a compact subgroup of $G$. The Ricci flow $(G / H, g(\cdot))$ was assumed to be $G$-invariant and exist for all positive time. In each case, it was shown that there are pointed diffeomorphisms $\left\{\phi_{s}\right\}_{s \in(0, \infty)}$ of $G / H$ so that the blowdown limit $g_{\infty}(t)=\lim _{s \rightarrow \infty} \frac{1}{s} \phi_{s}^{*} g(s t)$ exists and is one of the expanding solitons listed in Theorem 1.2.

Remark 2.5. As an aside, instead of looking at the rescaled Ricci flow metric $\hat{g}(t)=\frac{g(t)}{t}$, one could also consider the normalized Ricci flow solution, with constant volume. The normalized Ricci flow solution is useful in some settings but in our case we get more uniform results, in terms of the Thurston type, by looking at $\hat{g}$. For example, let $N$ be a truncated singly-cusped finite-volume hyperbolic 3manifold, as in Example 2.3. Let $\Sigma_{1}$ and $\Sigma_{2}$ be compact connected surfaces with one boundary component and negative Euler characteristic. Put $M_{1}=N \cup_{T^{2}}\left(S^{1} \times \Sigma_{1}\right)$ and $M_{2}=\left(S^{1} \times \Sigma_{1}\right) \cup_{T^{2}}\left(S^{1} \times \Sigma_{2}\right)$, where the gluing of $M_{2}$ is such that it not just a product $S^{1} \times\left(\Sigma_{1} \cup_{S^{1}} \Sigma_{2}\right)$. Under the unnormalized Ricci flow, one expects that $\operatorname{vol}\left(M_{1}, g(t)\right) \sim$ const. $t^{3 / 2}$, due to the hyperbolic piece, whereas $\operatorname{vol}\left(M_{2}, g(t)\right) \sim$ const. $t$. Then the normalized Ricci flow on $M_{1}$ should collapse its $S^{1} \times\left(\Sigma_{1}-\partial \Sigma_{1}\right)$ piece, while the normalized Ricci flow on $M_{2}$ should have a three-dimensional pointed limit on its $S^{1} \times\left(\Sigma_{1}-\partial \Sigma_{1}\right)$ piece. In contrast, the pointed Gromov-Hausdorff $\operatorname{limit}_{t \rightarrow \infty}\left(M_{i}, m_{i}, \hat{g}(t)\right)$, with an appropriate choice of basepoint $m_{i}$ in the $S^{1} \times \Sigma_{1}$ piece, should be $\Sigma_{1}-\partial \Sigma_{1}$ with a complete finite-volume metric of constant curvature $-\frac{1}{2}$, independent of $i \in\{1,2\}$. 


\section{Collapsing and Ricci flow}

In this section we give an overview, aimed for geometers, of the use of groupoids in collapsing theory. More details are in [38, Section 5] and references therein. We also show that under the hypotheses of Theorem 1.2, the manifold has a single geometric piece.

Suppose that $\left(M^{n}, g(\cdot)\right)$ is a type-III Ricci flow solution that exists for $t \in(1, \infty)$, i.e., there is some $K>0$ so that $\|\operatorname{Riem}(g(t))\|_{\infty} \leq \frac{K}{t}$ for all $t>1$. Then the rescaled metrics $\hat{g}(t)=\frac{g(t)}{t}$ have uniformly bounded sectional curvature. Even if the manifolds $(M, \hat{g}(t))$ are collapsing in the Gromov-Hausdorff sense, we would still like to take a limit as $t \rightarrow \infty$, in some way, of the $n$-dimensional geometry. To do so, it is natural to apply the Cheeger-Fukaya-Gromov theory of bounded curvature collapse to the Ricci flow.

A main technique in the Cheeger-Fukaya-Gromov theory is to work $O(n)$-equivariantly on the orthonormal frame bundle $F M$. This is not very convenient when dealing with Ricci flow, as the induced flow on $F M$ is complicated. For this reason, we use an older approach to collapsing with bounded sectional curvature, as described in Gromov's book [20], that deals directly with the manifold $M$.

Let $M$ be a complete $n$-dimensional Riemannian manifold with sectional curvatures bounded in absolute value by a positive number $K$. Given $r \in\left(0, \frac{1}{\sqrt{K}}\right)$ and $m \in M$, we can consider the Riemannian metric $\exp _{m}^{*} g$ on $B(0, r) \subset T_{m} M$.

Given a sequence of pointed complete $n$-dimensional Riemannian manifolds $\left\{\left(M_{i}, m_{i}\right)\right\}_{i=1}^{\infty}$ with sectional curvatures bounded in absolute value by $K$, there is a convergent subsequence of the pointed geometries $B(0, r) \subset T_{m_{i}} M_{i}$, whose limit is a $C^{1, \alpha}$-metric on an $n$-dimensional $r$-ball $\left(B_{\infty}, m_{\infty}\right)$. If one has uniform bounds of the form $\left\|\nabla^{k} \operatorname{Riem}\left(M_{i}\right)\right\|_{\infty} \leq C(k)$ then one can assume that the limit is a $C^{\infty}$. metric and the convergence is $C^{\infty}$.

Define an equivalence relation $\sim_{i}$ on $B\left(0, \frac{r}{3}\right) \subset T_{m_{i}} M_{i}$ by saying that $y \sim_{i} z$ if $\exp _{m_{i}}(y)=\exp _{m_{i}}(z)$. Then $B\left(m_{i}, \frac{r}{3}\right) \subset M_{i}$ equals $\left(B\left(0, \frac{r}{3}\right) \subset T_{m_{i}} M_{i}\right) / \sim_{i}$. The equivalence relation $\sim_{i}$ is the equivalence relation of a pseudogroup $\Gamma_{i}$ of local isometries on $B(0, r) \subset T_{m_{i}} M_{i}$, also called the fundamental pseudogroup $\pi_{1}\left(M_{i}, m_{i} ; r\right)$. One can take a convergent subsequence of the pseudogroups, in an appropriate sense, to obtain a limit pseudogroup $\Gamma_{\infty}$ of local isometries of $B_{\infty}$, which is a local Lie group. Furthermore, a neighborhood of the identity of $\Gamma_{\infty}$ is isomorphic to a neighborhood of the identity of a nilpotent Lie group. In particular, after passing to the subsequences, the pointed Gromov-Hausdorff limit of $\left\{B\left(m_{i}, \frac{r}{3}\right) \subset M_{i}\right\}_{i=1}^{\infty}$ is $\left(B\left(m_{\infty}, \frac{r}{3}\right) \subset B_{\infty}\right) / \Gamma_{\infty}$.

In this way one constructs a limiting $\frac{r}{3}$-ball. It has the drawback that it only describes the (lifted) geometry near the basepoints $m_{i}$. As one started with complete Riemannian manifolds, one would like to have a limiting object which in some sense is also complete. For example, suppose that $\left(M_{i}, m_{i}\right)=(M, m)$ for all $i$. The above 
process would produce the limiting ball $B\left(0, \frac{r}{3}\right) \subset T_{m} M$, with $\Gamma_{\infty}=\pi_{1}(M, m ; r)$. However, the limiting object should be all of $(M, m)$.

One way to construct a global limiting object would be to move the basepoints to other points inside of $B\left(0, \frac{r}{3}\right) \subset T_{m_{i}} M_{i}$, construct new limiting balls, repeat the process and glue all of the ensuing balls together in a coherent way. In order to formalize such a limiting object, the notion of a "Riemannian megafold" was introduced in [46]. This essentially consists of a pseudogroup of local isometries of a Riemannian manifold. Another formalization was given in [38], in which the limiting object is a Riemannian groupoid. A Riemannian groupoid is an étale groupoid with a Riemannian metric on its space of units, for which the local diffeomorphisms coming from groupoid elements are local isometries. Riemannian groupoids have been extensively discussed in the literature on foliation theory, as they describe the transverse structure of Riemannian foliations. For details we refer to Section 5 in [38] and references therein.

(We take this opportunity to make some corrections to [38]. The $\infty$ on p. 629, line 42 , should read $(0, \infty)$; the $[0,1]$ on p. 658 , line 15 , should read $[0,1)$.)

The upshot is that if $\left\{\left(M_{i}, m_{i}\right)\right\}_{i=1}^{\infty}$ is a sequence of pointed complete $n$-dimensional Riemannian manifolds, and if for every $k \in \mathbb{Z}^{\geq 0}$ and $R \in \mathbb{R}^{+}$there is some $C(k, R)<\infty$ so that for all $i$ we have $\left|\nabla^{k} \operatorname{Riem}\left(M_{i}\right)\right| \leq C(k, R)$ on $B\left(m_{i}, R\right) \subset$ $M_{i}$, then a subsequence converges smoothly to a pointed complete closed effective Hausdorff $n$-dimensional Riemannian groupoid $\left(\mathrm{S}_{\infty}, O_{x_{\infty}}\right)$ [38, Proposition 5.9]. This statement is essentially a reformulation of results of Cheeger, Fukaya and Gromov.

Let $\$$ be a complete closed effective Hausdorff Riemannian groupoid. It carries a certain locally constant sheaf $\underline{g}$ of finite dimensional Lie algebras on its space of units $\varsigma^{(0)}$. These Lie algebras act as germs of Killing vector fields on $\varsigma^{(0)}$. Elements of $\left(S\right.$ that are sufficiently close to the space of units $\Im^{(0)}$, in the 1-jet topology, appear in the image of the exponentials of small local sections of $\underline{g}$. In our case, the Lie algebras are nilpotent and there is no point $x \in G^{(0)}$ at which all of the corresponding Killing vector fields vanish simultaneously, unless $g=0$. We will say that $(5)$ is locally free if the isotropy groups $\varsigma_{x}^{x}$ are finite.

Remark 3.1. The locally constant sheaf $\mathfrak{g}$ is analogous to a pure Nil-structure in the sense of [3]; see [49] for a recent survey. It may seem surprising that we always get pure structures on our limiting spaces, since a manifold that collapses with bounded curvature generally carries a mixed Nil-structure if the diameter is not bounded during the collapse. The point is that we are considering a completely collapsed limit. In general, given $\epsilon, K, D>0$, there is a number $\delta=\delta(n, \epsilon, K, D)$ so that if $\|\operatorname{Riem}(M)\|_{\infty} \leq K$ then the fundamental pseudogroup $\pi_{1}(F M, p ; \delta)$ (which is represented by loops at $p$ with length less than $\delta$ ) can be continuously transported to any point $q \in B(p, D) \subset F M$, and the result maps into $\pi_{1}(F M, q ; \epsilon)$ 
[17, Lemma 7.2]. The fact that one generally cannot transport the short loops arbitrarily far, while keeping them short, is responsible for the appearance of mixed Nil-structures. As we are considering a completely collapsed limit, we can effectively move $\underline{\mathfrak{g}}_{p}$ to $\underline{\mathfrak{g}}_{q}$ for an arbitrary value of $D$.

The work of Cheeger-Fukaya-Gromov describes the local structure of a Riemannian manifold with bounded sectional curvature that is highly collapsed but not completely collapsed. The technique to do this, for example in [6], is to rescale the highly-collapsed manifold at a point $p$ in order to make the rescaled injectivity radius equal to 1 and the sectional curvatures very small. One then argues that the local geometry around $p$ is modeled on a complete flat $n$-dimensional manifold other than $\mathbb{R}^{n}$, giving the local $F$-structure. When dealing with Ricci flow this rescaling is problematic, as it does not mesh well with the flow. For this reason, we only deal with completely collapsed limits.

The notion of smooth pointed convergence of Riemannian groupoids is given in [38], which extends these collapsing considerations to the Ricci flow. (Related Ricci flow limits on a single ball in a tangent space were considered in [18].) In [38] the Ricci flow on an étale groupoid was considered. This consists of a Ricci flow $g(t)$ on the space of units $G^{(0)}$, in the usual sense, so that for each $t$ the local diffeomorphisms (arising from elements of $(5)$ act by isometries. One has the following compactness theorem.

Theorem 3.2 ([38], Theorem 5.12). Let $\left\{\left(M_{i}, p_{i}, g_{i}(\cdot)\right)\right\}_{i=1}^{\infty}$ be a sequence of Ricci flow solutions on pointed $n$-dimensional manifolds $\left(M_{i}, p_{i}\right)$. We assume that there are numbers $-\infty \leq A<0$ and $0<\Omega \leq \infty$ so that the following holds:

1. Each Ricci flow solution $\left(M_{i}, p_{i}, g_{i}(\cdot)\right)$ is defined on the time interval $(A, \Omega)$.

2. For each $t \in(A, \Omega), g_{i}(t)$ is a complete Riemannian metric on $M_{i}$.

3. For each compact interval $I \subset(A, \Omega)$ there is some $K_{I}<\infty$ so that

$$
\left|\operatorname{Riem}\left(g_{i}\right)(x, t)\right| \leq K_{I}
$$

for all $x \in M_{i}$ and $t \in I$.

Then after passing to a subsequence, the Ricciflow solutions $g_{i}(\cdot)$ converge smoothly to a Ricciflow solution $g_{\infty}(\cdot)$ on a pointed $n$-dimensional étale groupoid $\left(\mathfrak{F}_{\infty}, O_{x_{\infty}}\right)$, defined again for $t \in(A, \Omega)$.

This theorem is an analog of Hamilton's compactness theorem [26], except without the assumption of a uniform positive lower bound on the injectivity radius at $p_{i} \in\left(M_{i}, g_{i}(0)\right)$. In Hamilton's theorem one obtains a limiting Ricci flow on a manifold, which is a special type of étale groupoid. The proof of Theorem 5.12 in [38] is essentially the same as the proof of Hamilton's compactness theorem, when transplanted to the groupoid setting. 
Remark 3.3. If $\left\{\operatorname{diam}\left(M_{i}, g_{i}(0)\right)\right\}_{i=1}^{\infty}$ is uniformly bounded above then $\left(\varsigma_{\infty}, g_{\infty}(0)\right)$ has finite diameter and we do not have to talk about basepoints.

An immediate consequence of Theorem 3.2 is the following.

Corollary 3.4 ([38], Corollary 5.15). Given $K>0$, the space of pointed Ricci flow solutions on $n$-dimensional manifolds, with $\sup _{t \in(1, \infty)} t\left\|\operatorname{Riem}\left(g_{t}\right)\right\|_{\infty} \leq K$, is relatively compact among Ricci flows on pointed $n$-dimensional étale groupoids, defined for $t \in(1, \infty)$.

The next proposition will be used in later sections.

Proposition 3.5. Let $(M, g(\cdot))$ be a Ricci flow solution on a closed orientable 3-manifold that is defined for all $t \in[0, \infty)$. Suppose that

1. the sectional curvatures of $(M, g(t))$ are uniformly $O\left(t^{-1}\right)$, and

2. $\operatorname{diam}(M, g(t))=O\left(t^{\frac{1}{2}}\right)$.

Then $M$ is irreducible, aspherical and its geometric decomposition contains a single geometric piece.

Proof. As mentioned in Section 2, since the Ricci flow exists for all $t \in[0, \infty)$ it follows that $M$ is aspherical. The validity of the Poincare Conjecture then implies that $M$ is irreducible [41, Theorem 2].

Put $\hat{g}(t)=\frac{g(t)}{t}$. From the evolution equation for the scalar curvature $R$ and the maximum principle applied to $R+\frac{3}{2 t}$, it follows that $\operatorname{vol}(M, \hat{g}(t))$ is nonincreasing in $t$; see, for example, [14, (1.7)]. Suppose that $\lim _{t \rightarrow \infty} \operatorname{vol}(M, \hat{g}(t))>0$. Then $(M, \hat{g}(t))$ is noncollapsing. Recall Definition 1.1. If $\left\{s_{j}\right\}_{j=1}^{\infty}$ is a sequence tending to infinity then Hamilton's compactness theorem [26] implies that after passing to a subsequence, there is a limiting three-dimensional Ricci flow solution $\left(M_{\infty}, g_{\infty}(\cdot)\right)=\lim _{j \rightarrow \infty}\left(M, g_{s_{j}}(\cdot)\right)$. From the diameter assumption, $M_{\infty}$ is diffeomorphic to $M$. Using monotonic quantities, one can show that $\left(M_{\infty}, g_{\infty}(t)\right)$ has constant sectional curvature $-\frac{1}{4 t}$; see [14, Section 1] and references therein. Thus $M$ has an $H^{3}$-structure.

Now suppose that $\lim _{t \rightarrow \infty} \operatorname{vol}(M, \hat{g}(t))=0$. Then $(M, \hat{g}(t))$ collapses with bounded sectional curvature and bounded diameter. There will be a sequence $t_{i} \rightarrow \infty$ such that the Gromov-Hausdorff $\operatorname{limit}_{i \rightarrow \infty}\left(M, \hat{g}\left(t_{i}\right)\right)$ exists and equals some compact metric space $X$ of dimension less than three. In what follows we use some results about bounded curvature collapsing from [49] and references therein.

If $\operatorname{dim}(X)=0$ then $M$ is an almost flat manifold and so has an $\mathbb{R}^{3}$ or Nil-structure [19].

If $\operatorname{dim}(X)=2$ then $X$ is a closed orbifold and $M$ is the total space of an orbifold circle bundle over $X$ [16, Proposition 11.5], from which it follows that $M$ has a geometric structure. 
Finally, suppose that $\operatorname{dim}(X)=1$. First, $X$ is $S^{1}$ or an interval. If $X=S^{1}$ then $M$ is the total space of a torus bundle over $S^{1}$ and hence carries a geometric structure. If $X$ is an interval $[0, L]$ then there is a Gromov-Hausdorff approximation $\pi: M \rightarrow X$ with $\pi^{-1}(0, L)=(0, L) \times T^{2}$. Now $X$ is locally the quotient of $M$ by a fixed-point free $T^{2}$-action [5]. If the action is locally free then [0,L] is an orbifold. As the orbifold $[0, L]$ is double covered by $S^{1}$, the manifold $M$ is double covered by a $T^{2}$-bundle over $S^{1}$. Hence in this case, $M$ has a geometric structure [40].

Suppose that the $T^{2}$-action is not locally free, say on $\pi^{-1}[0, \delta)$, with $\delta$ small. From the slice theorem, a neighborhood of $\pi^{-1}(0)$ is equivariantly diffeomorphic to $T^{2} \times_{H} \mathbb{R}^{N}$, where $H$ is the isotropy group. As the $T^{2}$-action has no fixed points, $H$ must be a virtual circle group. However, since $M$ is aspherical, the map $\pi_{1}\left(T^{2}\right) \rightarrow$ $\pi_{1}(M)$ must be injective [7, Remark 0.9]. This is a contradiction. Similarly, the $T^{2}$-action must be locally free on $\pi^{-1}(L-\delta, L]$.

Remark 3.6. In our case, one can see directly that there is a contradiction if $H$ is a virtual circle group. Suppose so. Then $\pi^{-1}([0, \delta])\left(\right.$ or $\left.\pi^{-1}([L-\delta, L])\right)$ is diffeomorphic to $S^{1} \times D^{2}$. If the $T^{2}$-action fails to be locally free on both $\pi^{-1}([0, \delta])$ and $\pi^{-1}([L-\delta, L])$ then $M$ is the union of two solid tori and so is diffeomorphic to $S^{3}, S^{1} \times S^{2}$ or a lens space. If it fails to be locally free on exactly one of $\pi^{-1}([0, \delta])$ and $\pi^{-1}([L-\delta, L])$ then a double cover of $M$ is diffeomorphic to $S^{3}, S^{1} \times S^{2}$ or a lens space. In either case, $M$ fails to be aspherical.

Remark 3.7. By the argument of the proof of Proposition 3.5, we can say the following about aspherical 3-manifolds that collapse with bounded curvature and bounded diameter. If $M$ carries an $H^{3}$-structure then it cannot collapse. If $M$ carries an $H^{2} \times \mathbb{R}$ or $\widetilde{\mathrm{SL}_{2}(\mathbb{R})}$-structure then it can only collapse to a two-dimensional orbifold of negative Euler characteristic. If $M$ carries a Sol-structure then it can only collapse to $S^{1}$ or an interval. However, if $M$ carries an $\mathbb{R}^{3}$ or Nil-structure then a priori it could collapse to a two-dimensional orbifold with vanishing Euler characteristic, a circle, an interval or a point. We will show that under the Ricci flow, with our curvature and diameter assumptions it can only collapse to a point.

Remark 3.8. Some results about three-dimensional type-IIb Ricci flow solutions, i.e., Ricci flow solutions defined on $[0, \infty)$ with $\lim \sup _{t \rightarrow \infty} t\|\operatorname{Riem}(g(t))\|_{\infty}=\infty$, were obtained in [9]. Although phrased differently, the collapsing results in [9] can be considered to be results about Ricci flow solutions with nonnegative sectional curvature on three-dimensional étale groupoids. 


\section{Dimensional reduction}

In this section we consider a Ricci flow $(M, \bar{g}(\cdot))$ which is invariant under local actions of a connected abelian Lie group on $M$. We first define the notion of a twisted principal bundle and write out the Ricci flow equation for an invariant metric $\bar{g}$ on the total space $M$. The Ricci flow equation becomes as a coupled system of equations on the base $B$ of the twisted principal bundle. We construct modified $\mathscr{F}, \mathcal{W}$ and $\mathcal{W}_{+}$ functionals for $\bar{g}(\cdot)$ and show that they are monotonic. We use $\mathcal{W}_{+}$to show that any blowdown limit of $\bar{g}(\cdot)$ satisfies the harmonic-Einstein equations of [38].

Related functionals were considered independently by Bernhard List [37] and Jeff Streets [53]. (I thank Gerhard Huisken and Gang Tian for these references.) In [37] the modified $\mathcal{F}$-functional is considered in the special case when $N=1$ and $A_{\alpha}^{i}=0$. The motivation comes from the static Einstein equation. In [53], modified $\mathcal{F}$ and $\mathcal{W}$-functionals are considered for a certain invariant flow on the total space of a principal bundle, with the fiber geometry being fixed under the flow.

4.1. Twisted principal bundles. Let $\mathscr{E}$ be a Lie group, with Lie algebra g. Let $B$ be a connected $n$-dimensional smooth manifold. Let $\mathcal{E}$ be a local system on $B$ of Lie groups isomorphic to $\mathscr{G}$. Fixing a basepoint $b_{0} \in B$ and an isomorphism $\mathcal{E}_{b_{0}} \cong \mathscr{E}$ of the stalk over $b_{0}$, the local system is specified by a homomorphism $\rho: \pi_{1}(B, b) \rightarrow \operatorname{Aut}(\mathscr{E})$. Equivalently, we have a $\mathscr{E}$-bundle $E=\mathscr{E} \times_{\rho} \widetilde{B}$ over $B$, with a flat connection, which gives the étale space of the locally constant sheaf $\mathcal{E}$. Put $e=\mathfrak{g} \times{ }_{\rho} \widetilde{B}$, a flat $\mathrm{g}$-vector bundle on $B$. We will write $\Lambda^{\max } e=\Lambda^{\max } \mathrm{g} \times{ }_{\rho} \widetilde{B}$ for the corresponding flat real line bundle on $B$ of fiberwise volume forms, and $\left|\Lambda^{\max } e\right|=\left|\Lambda^{\max } \mathrm{g}\right| \times_{\rho} \widetilde{B}$ for the flat $\mathbb{R}^{\geq 0}$-bundle of fiberwise densities.

Hereafter we assume that the density bundle $\left|\Lambda^{\max } e\right|$ is a flat product bundle $\mathbb{R}^{\geq 0} \times B$. (Some of the subsequent results do not need this assumption, but for simplicity we will assume uniformly that it holds.)

Example 4.1. If $\mathscr{G}=\mathbb{R}^{N}$ then $\operatorname{Aut}(\mathscr{G})=\mathrm{GL}(N, \mathbb{R})$ and $E=e$ is a flat $\mathbb{R}^{N}$-bundle over $B$. The assumption on $\left|\Lambda^{\max } e\right|$ means that the holonomy of $e$ lies in $\operatorname{det}^{-1}( \pm 1)$. If $\mathscr{G}=T^{N}$ then $\operatorname{Aut}(\mathscr{E})=\mathrm{GL}(N, \mathbb{Z}), E$ is a flat $T^{N}$-bundle over $B$ and $e$ is a flat $\mathbb{R}^{N}$-bundle over $B$. In this case the assumption on $\left|\Lambda^{\max } e\right|$ holds automatically.

Let $\pi: M \rightarrow B$ be a fiber bundle with fiber $\mathcal{G}$. We write $E_{b}$ for the fiber of $E$ over $b \in B$ and $M_{b}$ for the fiber of $M$ over $b \in B$. Consider the fiber product $E \times{ }_{B} M=\bigcup_{b \in B} E_{b} \times M_{b}$. We assume that there is a smooth map $E \times_{B} M \rightarrow M$ so that over a point $b \in B$, the map $E_{b} \times M_{b} \rightarrow M_{b}$ gives a free transitive action of $\mathcal{E} \cong E_{b}$ on $M_{b}$. The action must be consistent with the flat connection on $E$ in the sense that if $U \subset B$ is such that $\left.E\right|_{U} \cong U \times \mathscr{E}$ is a local trivialization of the flat $\mathscr{E}$-bundle $E$ then $\pi^{-1}(U)$ has a free $\mathscr{E}$-action, and so is the total space of a 
principal $\mathscr{E}$-bundle over $U$. In this way, $M$ can be considered to be a twisted principal $\mathcal{E}$-bundle over $B$, with the twisting coming from the flat $\mathscr{E}$-bundle $E$. There is a natural isomorphism between the vertical tangent bundle $T^{\text {vert }} M=\operatorname{Ker}(d \pi)$ and $\pi^{*} e$.

An isomorphism of two twisted principal $\mathscr{E}$-bundles $\pi: M \rightarrow B$ and $\pi^{\prime}: M^{\prime} \rightarrow$ $B^{\prime}$ is given by a diffeomorphism $\eta: B \rightarrow B^{\prime}$, an isomorphism $\hat{\phi}: E \rightarrow E^{\prime}$ of flat $\mathcal{E}$-bundles that covers $\eta$, and a diffeomorphism $\phi: M \rightarrow M^{\prime}$ that covers $\eta$ with the property that for all $m \in M$ and $x \in E_{\pi(m)}$, we have $\phi(x \cdot m)=\widehat{\phi}(x) \cdot \phi(m)$.

It makes sense to talk about a connection $A \in \Omega^{1}\left(M ; \pi^{*} e\right)$ on a twisted principal $\mathscr{G}$-bundle $M$. The restriction of $A$ to $\pi^{-1}(U)$ is a $\mathrm{g}$-valued connection in the usual sense.

We assume that $M$ has a Riemannian metric $\bar{g}$ with a local free isometric $\mathscr{E}$-action. This means that if $\left.E\right|_{U} \cong U \times \mathscr{E}$ is a local trivialization of $E$ as above then the action of $\mathscr{E}$ on $\pi^{-1}(U)$ is isometric.

Hereafter we assume that $\mathscr{E}$ is a connected $N$-dimensional abelian Lie group.

Suppose that $U$ is also small enough so that $U$ is a coordinate chart for $B$ with local parametrization $\left\{x^{\alpha}\right\}_{\alpha=1}^{n} \rightarrow \rho\left(x^{\alpha}\right) \in U$. Take a section $s: U \rightarrow \pi^{-1}(U)$. Choosing a basis $\left\{e_{i}\right\}_{i=1}^{N}$ of $\mathfrak{g}$, we obtain coordinates on $\pi^{-1}(U)$ by $\left(x^{\alpha}, x^{i}\right) \rightarrow$ $\exp \left(\sum_{i=1}^{N} x^{i} e_{i}\right) \cdot s\left(\rho\left(x^{\alpha}\right)\right)$. In terms of these coordinates we can write

$$
\bar{g}=\sum_{i, j=1}^{N} G_{i j}\left(d x^{i}+A^{i}\right)\left(d x^{j}+A^{j}\right)+\sum_{\alpha, \beta=1}^{n} g_{\alpha \beta} d x^{\alpha} d x^{\beta} .
$$

Here $G_{i j}$ is the local expression of a Euclidean inner product on $e, A^{i}=\sum_{\alpha} A_{\alpha}^{i} d x^{\alpha}$ are the components of $s^{*} A$, and $\sum_{\alpha, \beta=1}^{n} g_{\alpha \beta} d x^{\alpha} d x^{\beta}$ is the local expression of a Riemannian metric $g_{B}$ on $B$. A change of section $s$ changes $A^{i}$ by an exact form. The curvatures $F^{i}=d A^{i}$ form an element of $\Omega^{2}(B ; e)$.

If $M$ and $M^{\prime}$ are two twisted principal $\mathcal{G}$-bundles then an isomorphism $\phi: M \rightarrow$ $M^{\prime}$ can be written in local coordinates as

$$
\phi\left(y^{\gamma}, y^{k}\right)=\left(x^{\alpha}\left(y^{\gamma}\right), \sum_{k} T_{k}^{i} y^{k}+f^{i}\left(y^{\gamma}\right)\right),
$$

where the $T_{k}^{i}$ 's are constants. It covers a diffeomorphism $\eta: B \rightarrow B^{\prime}$. The isomorphism $\hat{\phi}: E \rightarrow E^{\prime}$ of flat $\mathcal{G}$-bundles is represented locally by the functions $T^{i}{ }_{k}$.

A locally $\mathcal{G}$-invariant Ricci flow is a 1-parameter family of such Riemannian metrics $(M, \bar{g}(\cdot))$ that satisfies the Ricci flow equation. We will consider a basepoint for such a solution to be a point $p \in B$.

Let $\left\{\left(M_{i}, p_{i}, \bar{g}_{i}(\cdot)\right)\right\}_{i=1}^{\infty}$ be a sequence of locally $\mathscr{G}$-invariant Ricci flow solutions defined for $t \in(1, \infty)$. We say that $\lim _{i \rightarrow \infty}\left(M_{i}, p_{i}, \bar{g}_{i}(\cdot)\right)=\left(M_{\infty}, p_{\infty}, \bar{g}_{\infty}(\cdot)\right)$ if there are 
1. a sequence of open subsets $\left\{U_{j}\right\}_{j=1}^{\infty}$ of $B_{\infty}$, containing $p_{\infty}$, so that any compact subset of $B_{\infty}$ eventually lies in all $U_{j}$, and

2. open subsets $V_{i, j} \subset B_{i}$ containing $p_{i}$ and isomorphisms $\phi_{i, j}: \pi_{\infty}^{-1}\left(U_{j}\right) \rightarrow$ $\pi_{i}^{-1}\left(V_{i, j}\right)$ sending $\pi_{\infty}^{-1}\left(p_{\infty}\right)$ to $\pi_{i}^{-1}\left(p_{i}\right)$, so that

3. for all $j, \lim _{i \rightarrow \infty} \phi_{i, j}^{*} \bar{g}_{i}(\cdot)=\bar{g}_{\infty}(\cdot)$ smoothly on $\pi_{\infty}^{-1}\left(U_{j}\right) \times\left[1+j^{-1}, 1+j\right]$.

If $B_{\infty}$ is compact then we can remove the reference to basepoints.

4.2. Ricci flow on twisted principal bundles. In what follows, we use the Einstein summation convention freely. Let $\left(x^{\alpha}, x^{i}\right)$ be local coordinates on $\pi^{-1}(U)$ as in Section 4.1. Writing $A^{i}=\sum_{\alpha=1}^{n} A_{\alpha}^{i} d x^{\alpha}$, put $F_{\alpha \beta}^{i}=\partial_{\alpha} A_{\beta}^{i}-\partial_{\beta} A_{\alpha}^{i}$. We also write

$$
G_{i j ; \alpha \beta}=G_{i j, \alpha \beta}-\Gamma_{\alpha \beta}^{\sigma} G_{i j, \sigma},
$$

where $\left\{\Gamma_{\alpha \beta}^{\sigma}\right\}$ are the Christoffel symbols for the metric $g_{\alpha \beta}$ on $B$.

Given $b \in U$, it is convenient to choose the section $s$ so that $A^{i}(b)=0$. Then the curvature tensor $\bar{R}_{I J K L}$ of $M$ is given in terms of the curvature tensor $R_{\alpha \beta \gamma \delta}$ of $B$, the 2-forms $F_{\alpha \beta}^{i}$ and the metrics $G_{i j}$ by

$$
\begin{aligned}
\bar{R}_{i j k l}= & -\frac{1}{4} g^{\alpha \beta} G_{i k, \alpha} G_{j l, \beta}+\frac{1}{4} g^{\alpha \beta} G_{i l, \alpha} G_{j k, \beta}, \\
\bar{R}_{i j k \alpha}= & \frac{1}{4} g^{\beta \gamma} G_{j m} G_{i k, \beta} F_{\alpha \gamma}^{m}-\frac{1}{4} g^{\beta \gamma} G_{i m} G_{j k, \beta} F_{\alpha \gamma}^{m}, \\
\bar{R}_{i j \alpha \beta}= & -\frac{1}{4} G^{m k} G_{i m, \alpha} G_{k j, \beta}+\frac{1}{4} G^{m k} G_{i m, \beta} G_{k j, \alpha} \\
& \quad-\frac{1}{4} g^{\gamma \delta} G_{i m} G_{j k} F_{\alpha \gamma}^{m} F_{\beta \delta}^{k}+\frac{1}{4} g^{\gamma \delta} G_{i m} G_{j k} F_{\beta \gamma}^{m} F_{\alpha \delta}^{k}, \\
\bar{R}_{i \alpha j \beta}= & -\frac{1}{2} G_{i j ; \alpha \beta}+\frac{1}{4} G^{k l} G_{i k, \beta} G_{j l, \alpha}+\frac{1}{4} g^{\gamma \delta} G_{i k} G_{j l} F_{\alpha \gamma}^{k} F_{\beta \delta}^{l}, \\
\bar{R}_{i \alpha \beta \gamma}= & \frac{1}{2} G_{i j} F_{\beta \gamma ; \alpha}^{j}+\frac{1}{2} G_{i j, \alpha} F_{\beta \gamma}^{j}+\frac{1}{4} G_{i j, \beta} F_{\alpha \gamma}^{j}-\frac{1}{4} G_{i j, \gamma} F_{\alpha \beta}^{j}, \\
\bar{R}_{\alpha \beta \gamma \delta}= & R_{\alpha \beta \gamma \delta}-\frac{1}{2} G_{i j} F_{\alpha \beta}^{i} F_{\gamma \delta}^{j}-\frac{1}{4} G_{i j} F_{\alpha \gamma}^{i} F_{\beta \delta}^{j}+\frac{1}{4} G_{i j} F_{\alpha \delta}^{i} F_{\beta \gamma}^{j} .
\end{aligned}
$$

The Ricci tensor is given by

$$
\begin{aligned}
\bar{R}_{i j}= & -\frac{1}{2} g^{\alpha \beta} G_{i j ; \alpha \beta}-\frac{1}{4} g^{\alpha \beta} G^{k l} G_{k l, \alpha} G_{i j, \beta}+\frac{1}{2} g^{\alpha \beta} G^{k l} G_{i k, \alpha} G_{l j, \beta} \\
& \quad+\frac{1}{4} g^{\alpha \gamma} g^{\beta \delta} G_{i k} G_{j l} F_{\alpha \beta}^{k} F_{\gamma \delta}^{l}, \\
\bar{R}_{i \alpha}= & \frac{1}{2} g^{\gamma \delta} G_{i k} F_{\alpha \gamma ; \delta}^{k}+\frac{1}{2} g^{\gamma \delta} G_{i k, \gamma} F_{\alpha \delta}^{k}+\frac{1}{4} g^{\gamma \delta} G_{i m} G^{k l} G_{k l, \gamma} F_{\alpha \delta}^{m}, \\
\bar{R}_{\alpha \beta}= & R_{\alpha \beta}-\frac{1}{2} G^{i j} G_{i j ; \alpha \beta}+\frac{1}{4} G^{i j} G_{j k, \alpha} G^{k l} G_{l i, \beta}-\frac{1}{2} g^{\gamma \delta} G_{i j} F_{\alpha \gamma}^{i} F_{\beta \delta}^{j} .
\end{aligned}
$$

The scalar curvature is

$$
\begin{aligned}
\bar{R}=R & -g^{\alpha \beta} G^{i j} G_{i j ; \alpha \beta}+\frac{3}{4} g^{\alpha \beta} G^{i j} G_{j k, \alpha} G^{k l} G_{l i, \beta} \\
& -\frac{1}{4} g^{\alpha \beta} G^{i j} G_{i j, \alpha} G^{k l} G_{k l, \beta}-\frac{1}{4} g^{\alpha \gamma} g^{\beta \delta} G_{i j} F_{\alpha \beta}^{i} F_{\gamma \delta}^{j} .
\end{aligned}
$$


Consider a 1-parameter family of such Riemannian metrics $\bar{g}(\cdot)$ on $M$. Writing $G_{i j}(t), A_{\alpha}^{i}(t)$ and $g_{\alpha \beta}(t)$ as functions of $t$, the Ricci flow equation becomes

$$
\begin{aligned}
& \frac{d}{d t}\left(G_{i j}\left(d x^{i}+A^{i}\right)\left(d x^{j}+A^{j}\right)+g_{\alpha \beta} d x^{\alpha} d x^{\beta}\right) \\
& =-2 \bar{R}_{i j}\left(d x^{i}+A^{i}\right)\left(d x^{j}+A^{j}\right) \\
& \quad-4 \bar{R}_{i \alpha}\left(d x^{i}+A^{i}\right) d x^{\alpha}-2 \bar{R}_{\alpha \beta} d x^{\alpha} d x^{\beta} .
\end{aligned}
$$

Equivalently,

$$
\begin{aligned}
\frac{\partial G_{i j}}{\partial t}= & g^{\alpha \beta} G_{i j ; \alpha \beta}+\frac{1}{2} g^{\alpha \beta} G^{k l} G_{k l, \alpha} G_{i j, \beta}-g^{\alpha \beta} G^{k l} G_{i k, \alpha} G_{l j, \beta} \\
& \quad-\frac{1}{2} g^{\alpha \gamma} g^{\beta \delta} G_{i k} G_{j l} F_{\alpha \beta}^{k} F_{\gamma \delta}^{l}, \\
\frac{\partial A_{\alpha}^{i}}{\partial t}=- & g^{\gamma \delta} F_{\alpha \gamma ; \delta}^{i}-g^{\gamma \delta} G^{i j} G_{j k, \gamma} F_{\alpha \delta}^{k}-\frac{1}{2} g^{\gamma \delta} G^{k l} G_{k l, \gamma} F_{\alpha \delta}^{i}, \\
\frac{\partial g_{\alpha \beta}}{\partial t}=- & -2 R_{\alpha \beta}+G^{i j} G_{i j ; \alpha \beta}-\frac{1}{2} G^{i j} G_{j k, \alpha} G^{k l} G_{l i, \beta}+g^{\gamma \delta} G_{i j} F_{\alpha \gamma}^{i} F_{\beta \delta}^{j} .
\end{aligned}
$$

Adding a Lie derivative with respect to $-\nabla \ln \sqrt{\operatorname{det}\left(G_{i j}\right)}$ to the right-hand side, and adding an exact form to the right-hand side of the equation for $\frac{\partial A_{\alpha}^{i}}{\partial t}$, gives a new equivalent set of equations :

$$
\begin{aligned}
\frac{\partial G_{i j}}{\partial t} & =g^{\alpha \beta} G_{i j ; \alpha \beta}-g^{\alpha \beta} G^{k l} G_{i k, \alpha} G_{l j, \beta}-\frac{1}{2} g^{\alpha \gamma} g^{\beta \delta} G_{i k} G_{j l} F_{\alpha \beta}^{k} F_{\gamma \delta}^{l}, \\
\frac{\partial A_{\alpha}^{i}}{\partial t} & =-g^{\gamma \delta} F_{\alpha \gamma ; \delta}^{i}-g^{\gamma \delta} G^{i j} G_{j k, \gamma} F_{\alpha \delta}^{k}, \\
\frac{\partial g_{\alpha \beta}}{\partial t} & =-2 R_{\alpha \beta}+\frac{1}{2} G^{i j} G_{j k, \alpha} G^{k l} G_{l i, \beta}+g^{\gamma \delta} G_{i j} F_{\alpha \gamma}^{i} F_{\beta \delta}^{j} .
\end{aligned}
$$

The equations in (4.10) consist of a heat type equation for $G_{i j}$, a Yang-Mills gradient flow type equation for $A_{\alpha}^{i}$ and a Ricci flow type equation for $g_{\alpha \beta}$. If $B$ is closed then an extension of the DeTurck trick [13] to our setting shows short-time existence and uniqueness for the system (4.10).

4.2.1. Modified $\mathscr{F}$-functional. We now assume that $B$ is closed.

Definition 4.11. Given $f \in C^{\infty}(B)$, put

$$
\begin{array}{r}
\mathcal{F}\left(G_{i j}, A_{\alpha}^{i}, g_{\alpha \beta}, f\right)=\int_{B}\left(|\nabla f|^{2}+R-\frac{1}{4} g^{\alpha \beta} G^{i j} G_{j k, \alpha} G^{k l} G_{l i, \beta}\right. \\
\left.-\frac{1}{4} g^{\alpha \gamma} g^{\beta \delta} G_{i j} F_{\alpha \beta}^{i} F_{\gamma \delta}^{j}\right) e^{-f} \operatorname{dvol}_{B} .
\end{array}
$$

If $N=0$, i.e., if $M=B$, then this is the same as Perelman's $\mathcal{F}$-functional [43]. Otherwise, the expression in (4.12) differs from Perelman's $\mathcal{F}$-functional by 
the subtraction of terms corresponding to a Dirichlet energy of the field $G$ and a Yang-Mills action for the connection $A$.

We now compute the variation of $\mathcal{F}$.

Lemma 4.13. Given a smooth 1-parameter family

$$
\left\{\left(G_{i j}(s), A_{\alpha}^{i}(s), g_{\alpha \beta}(s), f(s)\right)\right\}_{s \in(-\epsilon, \epsilon)},
$$

write $\dot{G}_{i j}=\left.\frac{d G_{i j}}{d s}\right|_{s=0}, \dot{A}_{\alpha}^{i}=\left.\frac{d A_{\alpha}^{i}}{d s}\right|_{s=0}, \dot{g}_{\alpha \beta}=\left.\frac{d g_{\alpha \beta}}{d s}\right|_{s=0}$ and $\dot{f}=\left.\frac{d f}{d s}\right|_{s=0}$. Then

$$
\begin{aligned}
&\left.\frac{d}{d s}\right|_{s=0} \mathcal{F}\left(G_{i j}, A_{\alpha}^{i}, g_{\alpha \beta}, f\right) \\
&=-\int_{B} \dot{G}_{k l} G^{i k} G^{j l}\left(-\frac{1}{2} g^{\alpha \beta} G_{i j ; \alpha \beta}+\frac{1}{2} g^{\alpha \beta} G^{k l} G_{i k, \alpha} G_{l j, \beta}\right. \\
&\left.\quad+\frac{1}{4} g^{\alpha \gamma} g^{\beta \delta} G_{i k} G_{j l} F_{\alpha \beta}^{k} F_{\gamma \delta}^{l}+\frac{1}{2} g^{\alpha \beta} G_{i j, \alpha} f_{, \beta}\right) e^{-f} \mathrm{dvol}_{B} \\
&-2 \int_{B} \dot{A}_{\beta}^{j} g^{\alpha \beta} G_{i j}\left(\frac{1}{2} g^{\gamma \delta} F_{\alpha \gamma ; \delta}^{i}+\frac{1}{2} g^{\gamma \delta} G^{i j} G_{j k, \gamma} F_{\alpha \delta}^{k}\right. \\
&\left.-\frac{1}{2} g^{\gamma \delta} f_{, \gamma} F_{\alpha \delta}^{k}\right) e^{-f} \mathrm{dvol}_{B} \\
&-\int_{B} \dot{g}^{\alpha \beta}\left(R_{\alpha \beta}-\frac{1}{4} G^{i j} G_{j k, \alpha} G^{k l} G_{l i, \beta}\right. \\
&\left.-\frac{1}{2} g^{\gamma \delta} G_{i j} F_{\alpha \gamma}^{i} F_{\beta \delta}^{j}+f_{; \alpha \beta}\right) e^{-f} \mathrm{dvol}_{B} \\
&+\int_{B}\left(\frac{1}{2} g^{\alpha \beta} \dot{g}_{\alpha \beta}-\dot{f}\right)\left(2 \nabla^{2} f-|\nabla f|^{2}+R-\frac{1}{4} g^{\alpha \beta} G^{i j} G_{j k, \alpha} G^{k l} G_{l i, \beta}\right. \\
&\left.-\frac{1}{4} g^{\alpha \gamma} g^{\beta \delta} G_{i j} F_{\alpha \beta}^{i} F_{\gamma \delta}^{j}\right) e^{-f} \mathrm{dvol}_{B} .
\end{aligned}
$$

Proof. This follows from a calculation along the lines of the corresponding calculation for Perelman's $\mathcal{F}$-functional; see [31, Section 5].

We use the above lemma to show that $\mathcal{F}$ is nondecreasing under a certain flow.

Corollary 4.15. Under the flow equations

$$
\begin{aligned}
\frac{\partial G_{i j}}{\partial t}= & g^{\alpha \beta} G_{i j ; \alpha \beta}-g^{\alpha \beta} G^{k l} G_{i k, \alpha} G_{l j, \beta} \\
& -\frac{1}{2} g^{\alpha \gamma} g^{\beta \delta} G_{i k} G_{j l} F_{\alpha \beta}^{k} F_{\gamma \delta}^{l}-g^{\alpha \beta} G_{i j, \alpha} f_{, \beta}, \\
\frac{\partial A_{\alpha}^{i}}{\partial t}= & -g^{\gamma \delta} F_{\alpha \gamma ; \delta}^{i}-g^{\gamma \delta} G^{i j} G_{j k, \gamma} F_{\alpha \delta}^{k}+g^{\gamma \delta} f_{, \gamma} F_{\alpha \delta}^{k}, \\
\frac{\partial g_{\alpha \beta}}{\partial t}= & -2 R_{\alpha \beta}+\frac{1}{2} G^{i j} G_{j k, \alpha} G^{k l} G_{l i, \beta}+g^{\gamma \delta} G_{i j} F_{\alpha \gamma}^{i} F_{\beta \delta}^{j}-2 f_{; \alpha \beta}, \\
\frac{\partial f}{\partial t}= & -R+\frac{1}{4} g^{\alpha \beta} G^{i j} G_{j k, \alpha} G^{k l} G_{l i, \beta}+\frac{1}{2} g^{\alpha \gamma} g^{\beta \delta} G_{i j} F_{\alpha \beta}^{i} F_{\gamma \delta}^{j}-\nabla^{2} f
\end{aligned}
$$


one has

$$
\begin{aligned}
& \frac{d}{d t} \mathcal{F}\left(G_{i j}, A_{\alpha}^{i}, g_{\alpha \beta}, f\right) \\
& =\frac{1}{2} \int_{B} \mid g^{\alpha \beta} G_{i j ; \alpha \beta}-g^{\alpha \beta} G^{k l} G_{i k, \alpha} G_{l j, \beta} \\
& \quad-\frac{1}{2} g^{\alpha \gamma} g^{\beta \delta} G_{i k} G_{j l} F_{\alpha \beta}^{k} F_{\gamma \delta}^{l}-\left.g^{\alpha \beta} G_{i j, \alpha} f_{, \beta}\right|^{2} e^{-f} \mathrm{dvol}_{B} \\
& +\int_{B}\left|g^{\gamma \delta} F_{\alpha \gamma ; \delta}^{i}+g^{\gamma \delta} G^{i j} G_{j k, \gamma} F_{\alpha \delta}^{k}-g^{\gamma \delta} f_{, \gamma} F_{\alpha \delta}^{k}\right|^{2} e^{-f} \mathrm{dvol}_{B} \\
& +2 \int_{B} \mid R_{\alpha \beta}-\frac{1}{4} G^{i j} G_{j k, \alpha} G^{k l} G_{l i, \beta} \\
& \quad-\frac{1}{2} g^{\gamma \delta} G_{i j} F_{\alpha \gamma}^{i} F_{\beta \delta}^{j}+\left.f_{; \alpha \beta}\right|^{2} e^{-f} \mathrm{dvol}_{B} .
\end{aligned}
$$

Proof. This is an immediate consequence of Lemma 4.13.

As with Perelman's $\mathcal{F}$-functional, we now perform an infinitesimal diffeomorphism to decouple the equation for $f$ and obtain the Ricci flow on $M$.

Corollary 4.18. Under the flow equations

$$
\begin{aligned}
& \frac{\partial G_{i j}}{\partial t}=g^{\alpha \beta} G_{i j ; \alpha \beta}-g^{\alpha \beta} G^{k l} G_{i k, \alpha} G_{l j, \beta}-\frac{1}{2} g^{\alpha \gamma} g^{\beta \delta} G_{i k} G_{j l} F_{\alpha \beta}^{k} F_{\gamma \delta}^{l}, \\
& \frac{\partial A_{\alpha}^{i}}{\partial t}=-g^{\gamma \delta} F_{\alpha \gamma ; \delta}^{i}-g^{\gamma \delta} G^{i j} G_{j k, \gamma} F_{\alpha \delta}^{k}, \\
& \frac{\partial g_{\alpha \beta}}{\partial t}=-2 R_{\alpha \beta}+\frac{1}{2} G^{i j} G_{j k, \alpha} G^{k l} G_{l i, \beta}+g^{\gamma \delta} G_{i j} F_{\alpha \gamma}^{i} F_{\beta \delta}^{j}, \\
& \frac{\partial\left(e^{-f}\right)}{\partial t}=-\nabla^{2} e^{-f}+\left(R-\frac{1}{4} g^{\alpha \beta} G^{i j} G_{j k, \alpha} G^{k l} G_{l i, \beta}\right. \\
&\left.-\frac{1}{2} g^{\alpha \gamma} g^{\beta \delta} G_{i j} F_{\alpha \beta}^{i} F_{\gamma \delta}^{j}\right) e^{-f}
\end{aligned}
$$

one has

$$
\begin{aligned}
& \frac{d}{d t} \mathcal{F}\left(G_{i j}, A_{\alpha}^{i}, g_{\alpha \beta}, f\right) \\
& =\frac{1}{2} \int_{B} \mid g^{\alpha \beta} G_{i j ; \alpha \beta}-g^{\alpha \beta} G^{k l} G_{i k, \alpha} G_{l j, \beta} \\
& \quad-\frac{1}{2} g^{\alpha \gamma} g^{\beta \delta} G_{i k} G_{j l} F_{\alpha \beta}^{k} F_{\gamma \delta}^{l}-\left.g^{\alpha \beta} G_{i j, \alpha} f_{, \beta}\right|^{2} e^{-f} \mathrm{dvol}_{B}
\end{aligned}
$$




$$
\begin{aligned}
& +\int_{B}\left|g^{\gamma \delta} F_{\alpha \gamma ; \delta}^{i}+g^{\gamma \delta} G^{i j} G_{j k, \gamma} F_{\alpha \delta}^{k}-g^{\gamma \delta} f_{, \gamma} F_{\alpha \delta}^{k}\right|^{2} e^{-f} \mathrm{dvol}_{B} \\
& +2 \int_{B} \mid R_{\alpha \beta}-\frac{1}{4} G^{i j} G_{j k, \alpha} G^{k l} G_{l i, \beta} \\
& \quad-\frac{1}{2} g^{\gamma \delta} G_{i j} F_{\alpha \gamma}^{i} F_{\beta \delta}^{j}+\left.f_{; \alpha \beta}\right|^{2} e^{-f} \mathrm{dvol}_{B} .
\end{aligned}
$$

Proof. This follows because the right-hand sides of (4.16) and (4.19) differ by a Lie derivative with respect to $\nabla f$.

Note that the first three equations in (4.19) are the same as (4.10).

We now analyze what it means for $\mathcal{F}$ to be constant along the flow (4.19).

Proposition 4.21. If $\mathcal{F}\left(G_{i j}, A_{\alpha}^{i}, g_{\alpha \beta}, f\right)$ is constant in then $F_{\alpha \beta}^{i}=0, \operatorname{det}\left(G_{i j}\right)$ is constant and

$$
\begin{aligned}
g^{\alpha \beta} G_{i j ; \alpha \beta}-g^{\alpha \beta} G^{k l} G_{i k, \alpha} G_{l j, \beta} & =0, \\
R_{\alpha \beta}-\frac{1}{4} G^{i j} G_{j k, \alpha} G^{k l} G_{l i, \beta} & =0 .
\end{aligned}
$$

Proof. From (4.20), we have

$$
\begin{aligned}
& g^{\alpha \beta} G_{i j ; \alpha \beta}-g^{\alpha \beta} G^{k l} G_{i k, \alpha} G_{l j, \beta} \\
& \quad-\frac{1}{2} g^{\alpha \gamma} g^{\beta \delta} G_{i k} G_{j l} F_{\alpha \beta}^{k} F_{\gamma \delta}^{l}-g^{\alpha \beta} G_{i j, \alpha} f_{, \beta}=0
\end{aligned}
$$

and

$$
R_{\alpha \beta}-\frac{1}{4} G^{i j} G_{j k, \alpha} G^{k l} G_{l i, \beta} \frac{1}{2} g^{\gamma \delta} G_{i j} F_{\alpha \gamma}^{i} F_{\beta \delta}^{j}+f_{; \alpha \beta}=0 .
$$

Multiplying (4.23) by $G^{i j}$ and summing over indices gives

$$
\nabla^{2} \ln \operatorname{det}\left(G_{i j}\right)-\left\langle\nabla f, \nabla \ln \operatorname{det}\left(G_{i j}\right)\right\rangle-\frac{1}{2} g^{\alpha \gamma} g^{\beta \delta} G_{i j} F_{\alpha \beta}^{i} F_{\gamma \delta}^{j}=0 .
$$

(Here we are using the trivialization of $\left|\Lambda^{\max } e\right|$ to think of $\operatorname{det}\left(G_{i j}\right)$ as a function on $B$, defined up to multiplication by a positive constant.) Equivalently,

$$
\nabla^{\alpha}\left(e^{-f} \nabla_{\alpha} \ln \operatorname{det}\left(G_{i j}\right)\right)-\frac{1}{2} e^{-f} g^{\alpha \gamma} g^{\beta \delta} G_{i j} F_{\alpha \beta}^{i} F_{\gamma \delta}^{j}=0 .
$$

Integrating (4.26) over $B$ gives $F_{\alpha \beta}^{i}=0$. Then multiplying (4.26) by $\ln \operatorname{det}\left(G_{i j}\right)$ and integrating over $B$ gives $\nabla \ln \operatorname{det}\left(G_{i j}\right)=0$, so $\ln \operatorname{det}\left(G_{i j}\right)$ is spatially constant.

Given that $F_{\alpha \beta}^{i}=0$, the equation for $G^{i j} \frac{\partial G_{i j}}{\partial t}$ implies

$$
\frac{\partial}{\partial t} \ln \operatorname{det}\left(G_{i j}\right)=\nabla^{2} \ln \operatorname{det}\left(G_{i j}\right)
$$


Thus $\ln \operatorname{det}\left(G_{i j}\right)$ is also temporally constant.

As $\operatorname{det}\left(G_{i j}\right)$ is spatially constant, we have

$$
G^{i j} G_{i j ; \alpha \beta}-G^{i j} G_{j k, \alpha} G^{k l} G_{l i, \beta}=0 .
$$

Along with the fact that $F_{\alpha \beta}^{i}=0$, it follows that

$$
\begin{aligned}
\bar{R}_{i j} & =-\frac{1}{2} g^{\alpha \beta} G_{i j ; \alpha \beta}+\frac{1}{2} g^{\alpha \beta} G^{k l} G_{i k, \alpha} G_{l j, \beta} \\
\bar{R}_{i \alpha} & =0 \\
\bar{R}_{\alpha \beta} & =R_{\alpha \beta}-\frac{1}{4} G^{i j} G_{j k, \alpha} G^{k l} G_{l i, \beta}
\end{aligned}
$$

and

$$
\bar{R}=R-\frac{1}{4} g^{\alpha \beta} G^{i j} G_{j k, \alpha} G^{k l} G_{l i, \beta} .
$$

From equation (4.24),

$$
\int_{B} \bar{R} \mathrm{dvol}_{B}=0 .
$$

On $M$, the evolution of the scalar curvature is given by

$$
\frac{\partial \bar{R}}{\partial t}=\bar{\nabla}^{2} \bar{R}+2\left|\bar{R}_{I J}\right|^{2} .
$$

In our case, and using the fact that $\operatorname{det}\left(G_{i j}\right)$ is spatially constant, this becomes

$$
\frac{\partial \bar{R}}{\partial t}=\nabla^{2} \bar{R}+2\left|\bar{R}_{i j}\right|^{2}+2\left|\bar{R}_{\alpha \beta}\right|^{2} .
$$

From (4.19), (4.23) and (4.24), the flow equations are

$$
\begin{aligned}
\frac{\partial G_{i j}}{\partial t} & =g^{\alpha \beta} G_{i j, \alpha} f_{, \beta} \\
\frac{\partial g_{\alpha \beta}}{\partial t} & =2 f_{; \alpha \beta} .
\end{aligned}
$$

As the right-hand side of (4.34) is given by Lie derivatives with respect to $\nabla f$, it follows that

$$
\frac{\partial \bar{R}}{\partial t}=\langle\nabla f, \nabla \bar{R}\rangle
$$

Thus

$$
\nabla^{2} \bar{R}+2\left|\bar{R}_{i j}\right|^{2}+2\left|\bar{R}_{\alpha \beta}\right|^{2}=\langle\nabla f, \nabla \bar{R}\rangle,
$$

or

$$
\nabla^{2} \bar{R}+2\left|\bar{R}_{i j}\right|^{2}+2\left|\bar{R}_{\alpha \beta}-\frac{1}{n} \bar{R} g_{\alpha \beta}\right|^{2}+\frac{2}{n} \bar{R}^{2}=\langle\nabla f, \nabla \bar{R}\rangle .
$$

From (4.31), either $\bar{R}=0$ or $\bar{R}_{\text {min }}<0$. If $\bar{R}_{\text {min }}<0$ then we obtain a contradiction to the minimum principle, applied to (4.37). Thus $\bar{R}=0$. Equation (4.37) now implies that $\bar{R}_{i j}=\bar{R}_{\alpha \beta}=0$, which proves the proposition. 
From (4.19), under the conclusion of Proposition 4.21 it follows that $G_{i j}, A_{\alpha}^{i}$ and $g_{\alpha \beta}$ are time-independent. The Ricci flow solution $\bar{g}_{\infty}(\cdot)$ on $M$ is Ricci-flat. In the case $N=0$ the proof of Proposition 4.21 essentially reduces to the standard proof that a steady gradient soliton on a compact manifold is Ricci-flat; see, for example, Chapter 1 in [8].

With $\operatorname{det}^{-1}( \pm 1) \subset \mathrm{GL}(N, \mathbb{R})$, we can write

$$
\operatorname{det}^{-1}( \pm 1) / \mathrm{O}(N)=\operatorname{SL}(N, \mathbb{R}) / \operatorname{SO}(N) .
$$

From [38, Proposition 4.17], the first equation in (4.22) says that the map $b \rightarrow G_{i j}(b)$ describes a (twisted) harmonic map $G: B \rightarrow \operatorname{det}^{-1}( \pm 1) / \mathrm{O}(N)$. The twisting refers to the fact that if the flat $\mathbb{R}^{N}$-bundle $e$ has holonomy representation $\rho: \pi_{1}\left(B, b_{0}\right) \rightarrow$ $\operatorname{det}^{-1}( \pm 1)$ then we really have a harmonic map $\widetilde{G}: \widetilde{B} \rightarrow \operatorname{det}^{-1}( \pm 1) / \mathrm{O}(N)$ which satisfies $\widetilde{G}(\gamma \tilde{b})=\rho(\gamma) \widetilde{G}(\tilde{b})$ for $\gamma \in \pi_{1}(B, b)$ and $\tilde{b}$ in the universal cover $\widetilde{B}$. After passing to a double cover of $B$ if necessary, we can assume that $\rho$ takes value in $\operatorname{SL}(N, \mathbb{R})$. For simplicity, we will make this assumption hereafter and consider $\widetilde{G}$ to be a twisted harmonic map from $B$ to $\operatorname{SL}(N, \mathbb{R}) / \operatorname{SO}(N)$. Information on such twisted harmonic maps appears in [12], [30, Section 1.2] and [36]. Given $\rho$, such a twisted harmonic map $G$ exists if and only if the Zariski closure of $\operatorname{Im}(\rho)$ is reductive in $\operatorname{SL}(N, \mathbb{R})$. Given $\rho$, if there are two such equivariant harmonic maps $\widetilde{G}_{1}$ and $\widetilde{G}_{2}$ then there is a 1-parameter family $\left\{\widetilde{G}_{t}\right\}_{t \in[1,2]}$ of such equivariant harmonic maps, all with the same quotient energy, so that for each $\tilde{b} \in \widetilde{B}$ the map $t \rightarrow \widetilde{G}_{t}(\tilde{b})$ is a constant-speed geodesic arc, whose length is independent of $\tilde{b}$.

If the second equation in (4.22) is satisfied then $B$ clearly has nonnegative Ricci curvature.

We now look at the solutions of (4.22).

Proposition 4.38. Any solution $\bar{g}$ of (4.22) is a locally product metric on a Ricci-flat base B.

Proof. From the second equation in (4.22), $B$ has nonnegative Ricci curvature. For some $r$, the universal cover $\widetilde{B}$ is an isometric product of $\mathbb{R}^{r}$ and $W$, where $W$ is a simply-connected closed $(n-r)$-dimensional manifold of nonnegative Ricci curvature [4]. As before, let $\widetilde{G}: \widetilde{B} \rightarrow \operatorname{SL}(N, \mathbb{R}) / \operatorname{SO}(N)$ denote the lift of $G$ to $\widetilde{B}$.

Let $x^{1}, \ldots, x^{r}$ be Cartesian coordinates on $\mathbb{R}^{r}$ and let $x^{r+1}, \ldots, x^{n}$ be local coordinates on $W$. From the second equation of (4.22), $\widetilde{G}_{i j, \alpha}=0$ for $1 \leq \alpha \leq r$. That is, $\widetilde{G}$ is constant in the $\mathbb{R}^{k}$-directions. Then the first equation of (4.22) implies that for each $y \in \mathbb{R}^{r}$, the restriction of $\widetilde{G}$ to $\{y\} \times W$ is a harmonic map from $W$ to $\operatorname{SL}(N, \mathbb{R}) / \operatorname{SO}(N)$. It follows that for each $y \in \mathbb{R}^{r}$, the restriction of $\widetilde{G}$ to $\{y\} \times W$ is a point map. Thus $\widetilde{G}$ is constant. From the second equation of (4.22), $\widetilde{B}$ is Ricci flat. The conclusion is that $B$ is Ricci flat and $G$ is locally constant. 
In the next proposition we use $\mathcal{F}$ to analyze a long-time limit of a locally $\mathcal{G}$ invariant Ricci flow solution. The method of proof is along the lines of the proof of Theorem 1.3 in [14].

Proposition 4.39. Suppose that $(M, \bar{g}(\cdot))$ is a locally $\mathcal{G}$-invariant Ricci flow defined for all $t \in[0, \infty)$. Let $\left\{s_{i}\right\}_{i=1}^{\infty}$ be a sequence of positive numbers tending to infinity. Put $\bar{g}_{i}(t)=\bar{g}\left(t+s_{i}\right)$. Suppose that $\lim _{i \rightarrow \infty} \bar{g}_{i}(\cdot)$ exists and equals $\bar{g}_{\infty}(\cdot)$ in the

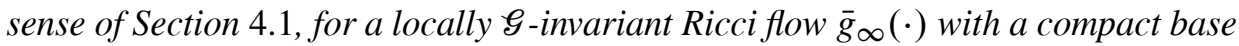
$B_{\infty}$. Writing $\bar{g}_{\infty}(\cdot) \equiv\left(G_{i j, \infty}(\cdot), A_{\alpha, \infty}^{i}(\cdot), g_{\alpha \beta, \infty}(\cdot)\right)$, we conclude that

1. the curvatures $F_{\alpha \beta, \infty}^{i}$ vanish;

2. $\operatorname{det}\left(G_{i j, \infty}\right)$ is constant;

3. equations (4.22) are satisfied for $G_{i j, \infty}(\cdot)$ and $g_{\alpha \beta, \infty}(\cdot)$.

Proof. We first construct a positive solution of the conjugate heat equation

$$
\frac{\partial u}{\partial t}=-\nabla^{2} u+\left(R-\frac{1}{4} g^{\alpha \beta} G^{i j} G_{j k, \alpha} G^{k l} G_{l i, \beta}-\frac{1}{2} g^{\alpha \gamma} g^{\beta \delta} G_{i j} F_{\alpha \beta}^{i} F_{\gamma \delta}^{j}\right) u .
$$

that exists for all $t \in[0, \infty)$. Note that if $u$ is a solution to (4.40) then $\int_{B} u \operatorname{dvol}_{B}$ is constant in $t$. Let $\left\{t_{j}\right\}_{j=1}^{\infty}$ be a sequence of times going to infinity. Let $\tilde{u}_{j}(\cdot)$ be a solution to (4.40) on the interval $\left[0, t_{j}\right]$ with initial condition $\tilde{u}_{j}\left(t_{j}\right)=\frac{1}{\operatorname{vol}\left(B, g_{\alpha \beta}\left(t_{j}\right)\right)}$. For any $T>0$, we claim that a subsequence of the $\tilde{u}_{j}$ 's converges smoothly on the time interval $[0, T]$. To see this, at time $T+1$ we know that if $t_{j} \geq T+1$ then $\tilde{u}_{j}(T+1) \geq 0$ and $\int_{B} \tilde{u}_{j}(T+1) \operatorname{dvol}_{B}=1$. Solving the conjugate heat equation with initial data at time $T+1$, and restricting the solution to the time interval $[0, T]$, gives a smoothing operator from the space of initial data $\left\{\tilde{u} \in L^{1}(B)\right.$ : $\left.\tilde{u} \geq 0, \int_{B} \tilde{u} \operatorname{dvol}_{B}(T+1)=1\right\}$ to $C^{\infty}([0, T] \times B)$. Thus we have the derivative bounds needed to extract a subsequence of the $\tilde{u}_{j}$ 's that converges smoothly on $[0, T]$. By a diagonal argument, we can extract a subsequence of the $\tilde{u}_{j}$ 's that converges smoothly on compact subsets of $[0, \infty)$ to a nonzero solution $\tilde{u}_{\infty}(\cdot)$ of $(4.40)$, defined for $t \in[0, \infty)$.

One can show, as in the proof of Proposition 7.5 in [31], that $\tilde{u}_{\infty}(\cdot)>0$. If $\tilde{f}_{\infty}(t)$ is given by $\tilde{u}_{\infty}(t)=e^{-\tilde{f}_{\infty}(t)}$ then $\mathcal{F}\left(G_{i j}(t), A_{\alpha}^{i}(t), g_{\alpha \beta}(t), \tilde{f}_{\infty}(t)\right)$ is nondecreasing in $t$. We write $\mathscr{F}_{\infty}=\lim _{t \rightarrow \infty} \mathscr{F}\left(G_{i j}(t), A_{\alpha}^{i}(t), g_{\alpha \beta}(t), \tilde{f}_{\infty}(t)\right)$, which is possibly infinite for the moment.

Next, put $u_{i}(t)=\tilde{u}_{\infty}\left(t+s_{i}\right)$. By assumption, $\lim _{i \rightarrow \infty} \bar{g}_{i}(\cdot)=\bar{g}_{\infty}(\cdot)$ in the sense of Section 4.1. Then by the same smoothing argument as above, there is a subsequence of $\left\{u_{i}(\cdot)\right\}_{i=1}^{\infty}$ that converges smoothly on compact subsets of $[0, \infty)$ to a solution $u_{\infty}(\cdot)$ of (4.40) on $B_{\infty}$, where (4.40) is now written in terms of $G_{i j, \infty}(\cdot)$, $A_{\alpha, \infty}^{i}(\cdot)$ and $g_{\alpha \beta, \infty}(\cdot)$. (When taking a convergent subsequence, we perform the 
same diffeomorphisms on the $u_{i}$ 's as are used in forming the $\operatorname{limit}_{i \rightarrow \infty} \lim _{i}(\cdot)$.) Define $f_{\infty}(t)$ by $u_{\infty}(t)=e^{-f_{\infty}(t)}$. Then after passing to a subsequence,

$$
\begin{aligned}
& \mathscr{F}\left(G_{i j, \infty}(t), A_{\alpha, \infty}^{i}(t), g_{\alpha \beta, \infty}(t), f_{\infty}(t)\right) \\
& \quad=\lim _{i \rightarrow \infty} \mathscr{F}\left(G_{i j}\left(t+s_{i}\right), A_{\alpha}^{i}\left(t+s_{i}\right), g_{\alpha \beta}\left(t+s_{i}\right), \tilde{f}_{\infty}\left(t+s_{i}\right)\right) \\
& \quad=\mathscr{F}_{\infty} .
\end{aligned}
$$

This shows that $\mathscr{F}_{\infty}<\infty$ and that $\mathcal{F}\left(G_{i j, \infty}(t), A_{\alpha, \infty}^{i}(t), g_{\alpha \beta, \infty}(t), f_{\infty}(t)\right)$ is constant in $t$. The proposition now follows from Proposition 4.21.

Junfang Li pointed out that the modified $\mathscr{F}$-functional has an $(n+N)$-dimensional interpretation. Namely, for $\bar{f} \in C^{\infty}(B)$, put

$$
\overline{\mathcal{F}}\left(G_{i j}, A_{\alpha}^{i}, g_{\alpha \beta}, \bar{f}\right)=\int_{B}\left(|\nabla \bar{f}|^{2}+\bar{R}\right) e^{-\bar{f}} \sqrt{\operatorname{det}\left(G_{i j}\right)} \operatorname{dvol}_{B} .
$$

This is a renormalized version of Perelman's $\mathscr{F}$-functional on $M$.

Proposition 4.43. Put $f=\bar{f}-\ln \sqrt{\operatorname{det}\left(G_{i j}\right)}$. Then

$$
\overline{\mathcal{F}}\left(G_{i j}, A_{\alpha}^{i}, g_{\alpha \beta}, \bar{f}\right)=\mathscr{F}\left(G_{i j}, A_{\alpha}^{i}, g_{\alpha \beta}, f\right) .
$$

Proof. We have

$$
\int_{B}|\nabla \bar{f}|^{2} e^{-\bar{f}} \sqrt{\operatorname{det}\left(G_{i j}\right)} \operatorname{dvol}_{B}=\int_{B}\left|\nabla f+\nabla \ln \sqrt{\operatorname{det}\left(G_{i j}\right)}\right|^{2} e^{-f} \operatorname{dvol}_{B}
$$

and

$$
\begin{gathered}
\int_{B}\left|\nabla f+\nabla \ln \sqrt{\operatorname{det}\left(G_{i j}\right)}\right|^{2} e^{-f} \operatorname{dvol}_{B} \\
=\int_{B}\left(|\nabla f|^{2}+2\left\langle\nabla f, \nabla \ln \sqrt{\operatorname{det}\left(G_{i j}\right)}\right\rangle+\left|\nabla \ln \sqrt{\operatorname{det}\left(G_{i j}\right)}\right|^{2}\right) e^{-f} \operatorname{dvol}_{B} \\
=\int_{B}\left(|\nabla f|^{2}+2 \nabla^{2} \ln \sqrt{\operatorname{det}\left(G_{i j}\right)}+\left|\nabla \ln \sqrt{\operatorname{det}\left(G_{i j}\right)}\right|^{2}\right) e^{-f} \operatorname{dvol}_{B} \\
=\int_{B}\left(|\nabla f|^{2}+g^{\alpha \beta} G^{i j} G_{i j ; \alpha \beta}-g^{\alpha \beta} G^{i j} G_{j k, \alpha} G^{k l} G_{l i, \beta}\right. \\
\left.\quad+\frac{1}{4} g^{\alpha \beta} G^{i j} G_{i j, \alpha} G^{k l} G_{k l, \beta}\right) e^{-f} \operatorname{dvol}_{B} .
\end{gathered}
$$


Combining this with (4.7) gives

$$
\begin{gathered}
\int_{B}\left(\left|\nabla f+\nabla \ln \sqrt{\operatorname{det}\left(G_{i j}\right)}\right|^{2}+\bar{R}\right) e^{-f} \mathrm{dvol}_{B} \\
=\int_{B}\left(|\nabla f|^{2}+R-\frac{1}{4} g^{\alpha \beta} G^{i j} G_{j k, \alpha} G^{k l} G_{l i, \beta}\right. \\
\left.-\frac{1}{4} g^{\alpha \gamma} g^{\beta \delta} G_{i j} F_{\alpha \beta}^{i} F_{\gamma \delta}^{j}\right) e^{-f} \operatorname{dvol}_{B},
\end{gathered}
$$

which proves the proposition.

\subsubsection{Modified W-functional}

Definition 4.48. Given $f \in C^{\infty}(B)$ and $\tau \in \mathbb{R}^{+}$, put

$$
\begin{aligned}
& \mathcal{W}\left(G_{i j}, A_{\alpha}^{i}, g_{\alpha \beta}, f, \tau\right) \\
& =\int_{B}\left[\tau \left(|\nabla f|^{2}+R-\frac{1}{4} g^{\alpha \beta} G^{i j} G_{j k, \alpha} G^{k l} G_{l i, \beta}\right.\right. \\
& \left.\left.\quad-\frac{1}{4} g^{\alpha \gamma} g^{\beta \delta} G_{i j} F_{\alpha \beta}^{i} F_{\gamma \delta}^{j}\right)+f-n\right](4 \pi \tau)^{-\frac{n}{2}} e^{-f} \operatorname{dvol}_{B} .
\end{aligned}
$$

If $N=0$, i.e., if $M=B$, then this is the same as Perelman's $\mathcal{W}$-functional [43]. The next proposition says how $\mathcal{W}$ varies along the Ricci flow.

Proposition 4.50. Under the flow equations

$$
\begin{aligned}
\frac{\partial G_{i j}}{\partial t} & =g^{\alpha \beta} G_{i j ; \alpha \beta}-g^{\alpha \beta} G^{k l} G_{i k, \alpha} G_{l j, \beta}-\frac{1}{2} g^{\alpha \gamma} g^{\beta \delta} G_{i k} G_{j l} F_{\alpha \beta}^{k} F_{\gamma \delta}^{l}, \\
\frac{\partial A_{\alpha}^{i}}{\partial t} & =-g^{\gamma \delta} F_{\alpha \gamma ; \delta}^{i}-g^{\gamma \delta} G^{i j} G_{j k, \gamma} F_{\alpha \delta}^{k}, \\
\frac{\partial g_{\alpha \beta}}{\partial t} & =-2 R_{\alpha \beta}+\frac{1}{2} G^{i j} G_{j k, \alpha} G^{k l} G_{l i, \beta}+g^{\gamma \delta} G_{i j} F_{\alpha \gamma}^{i} F_{\beta \delta}^{j} \\
\frac{\partial\left(e^{-f}\right)}{\partial t} & =-\nabla^{2} e^{-f}+\left(R-\frac{1}{4} g^{\alpha \beta} G^{i j} G_{j k, \alpha} G^{k l} G_{l i, \beta}\right. \\
\frac{\partial \tau}{\partial t} & =-1
\end{aligned}
$$


one has

$$
\begin{aligned}
& \frac{d}{d t} \mathcal{W}\left(G_{i j}, A_{\alpha}^{i}, g_{\alpha \beta}, f, \tau\right) \\
& =\frac{\tau}{2} \int_{B} \mid g^{\alpha \beta} G_{i j ; \alpha \beta}-g^{\alpha \beta} G^{k l} G_{i k, \alpha} G_{l j, \beta} \\
& -\frac{1}{2} g^{\alpha \gamma} g^{\beta \delta} G_{i k} G_{j l} F_{\alpha \beta}^{k} F_{\gamma \delta}^{l}-\left.g^{\alpha \beta} G_{i j, \alpha} f_{, \beta}\right|^{2}(4 \pi \tau)^{-\frac{n}{2}} e^{-f} \operatorname{dvol}_{B} \\
& +\tau \int_{B}\left|g^{\gamma \delta} F_{\alpha \gamma ; \delta}^{i}+g^{\gamma \delta} G^{i j} G_{j k, \gamma} F_{\alpha \delta}^{k}-g^{\gamma \delta} f_{, \gamma} F_{\alpha \delta}^{k}\right|^{2}(4 \pi \tau)^{-\frac{n}{2}} e^{-f} \operatorname{dvol}_{B} \\
& +2 \tau \int_{B} \mid R_{\alpha \beta}-\frac{1}{4} G^{i j} G_{j k, \alpha} G^{k l} G_{l i, \beta}-\frac{1}{2} g^{\gamma \delta} G_{i j} F_{\alpha \gamma}^{i} F_{\beta \delta}^{j} \\
& +f_{; \alpha \beta}-\left.\frac{1}{2 \tau} g_{\alpha \beta}\right|^{2}(4 \pi \tau)^{-\frac{n}{2}} e^{-f} \mathrm{dvol}_{B} \\
& -\frac{1}{4} \int_{B} g^{\alpha \gamma} g^{\beta \delta} G_{i j} F_{\alpha \beta}^{i} F_{\gamma \delta}^{j}(4 \pi \tau)^{-\frac{n}{2}} e^{-f} \operatorname{dvol}_{B} \text {. }
\end{aligned}
$$

Proof. The proof stands in relation to the proof of Corollary 4.18 as the corresponding statements about Perelman's W-functional vs. Perelman's $\mathcal{F}$-functional; see [31, Section 12].

Note that the $\frac{1}{4} \int_{B} g^{\alpha \gamma} g^{\beta \delta} G_{i j} F_{\alpha \beta}^{i} F_{\gamma \delta}^{j}(4 \pi \tau)^{-\frac{n}{2}} e^{-f}$ dvol $_{B}$ term occurs on the right-hand side of (4.52) with a negative sign. We now look at what it means for $\mathcal{W}$ to be constant in $t$, under the assumption that $F_{\alpha \beta}^{i}$ vanishes.

Proposition 4.53. Suppose that $F_{\alpha \beta}^{i}=0$. If $\mathcal{W}\left(G_{i j}, A_{\alpha}^{i}, g_{\alpha \beta}, f, \tau\right)$ is constant in $t$ then $\operatorname{det}\left(G_{i j}\right)$ is constant and

$$
\begin{aligned}
g^{\alpha \beta} G_{i j ; \alpha \beta}-g^{\alpha \beta} G^{k l} G_{i k, \alpha} G_{l j, \beta}-g^{\alpha \beta} G_{i j, \alpha} f_{, \beta} & =0, \\
R_{\alpha \beta}-\frac{1}{4} G^{i j} G_{j k, \alpha} G^{k l} G_{l i, \beta}+f_{; \alpha \beta}-\frac{1}{2 \tau} g_{\alpha \beta} & =0 .
\end{aligned}
$$

Proof. The same argument as in the proof of Proposition $4.21 \operatorname{shows}$ that $\operatorname{det}\left(G_{i j}\right)$ is constant. Then (4.54) follows from (4.52).

Unlike in Proposition 4.21, we cannot conclude that $f$ is constant, because of the existence of nontrivial compact gradient shrinking solitons.

Remark 4.55. The term $\frac{1}{4} \int_{B} g^{\alpha \gamma} g^{\beta \delta} G_{i j} F_{\alpha \beta}^{i} F_{\gamma \delta}^{j}(4 \pi \tau)^{-\frac{n}{2}} e^{-f} \operatorname{dvol}_{B}$ occurs on the right-hand side of (4.52) with a useless sign. This is not surprising, as can be seen by looking at the Ricci flow on a round 3 -sphere $M$, which we consider to be the total space of a circle bundle over $S^{2}$. We shift the time parameter so that the 3sphere disappears at time zero. As the 3 -sphere gives a gradient shrinking soliton, the functional $\mathcal{W}$ is constant in $t$. However, the circle bundle has nonvanishing curvature. Hence having $\mathcal{W}$ constant in $t$ cannot imply that $F_{\alpha \beta}^{i}$ vanishes. 
We now look at some special cases of (4.54).

Proposition 4.56. Under the hypotheses of Proposition 4.53, if $1 \leq \operatorname{dim}(B) \leq 2$ then the only solutions of (4.54) occur when $B$ is $S^{2}$ or $\mathbb{R} P^{2}$.

Proof. The second equation in (4.54) implies that

$$
\int_{B} R \operatorname{dvol}_{B}-\frac{1}{4} \int_{B} g^{\alpha \beta} G^{i j} G_{j k, \alpha} G^{k l} G_{l i, \beta} \operatorname{dvol}_{B}-\frac{n}{2 \tau} \operatorname{vol}(B)=0,
$$

from which the proposition follows.

We now use $\mathcal{W}$ to analyze a blowup limit.

Proposition 4.58. Suppose that $(M, \bar{g}(\cdot))$ is a locally $\mathcal{G}$-invariant Ricciflow defined for all $t \in(-T, 0)$, with $T \leq \infty$. Suppose that $F_{\alpha \beta}^{i}=0$. Put $\tau=-t$. Let $\left\{s_{i}\right\}_{i=1}^{\infty}$ be a sequence of positive numbers tending to infinity. Put $\bar{g}_{i}(\tau)=s_{i} \bar{g}\left(s_{i}^{-1} \tau\right)$. Suppose that $\lim _{i \rightarrow \infty} \bar{g}_{i}(\cdot)$ exists and equals $\bar{g}_{\infty}(\cdot)$ in the sense of Section 4.1, for a locally

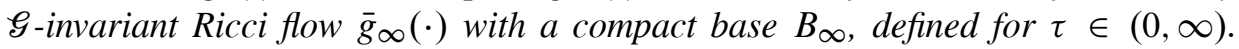
Writing $\bar{g}_{\infty}(\cdot) \equiv\left(G_{i j, \infty}(\cdot), g_{\alpha \beta, \infty}(\cdot)\right)$, we conclude that

1. $\operatorname{det}\left(G_{i j, \infty}\right)$ is constant;

2. equations (4.54) are satisfied for $G_{i j, \infty}(\cdot)$ and $g_{\alpha \beta, \infty}(\cdot)$.

Proof. The proof is along the lines of the proof of Proposition 4.39.

\subsubsection{Modified $\mathcal{W}_{+}$-functional}

Definition 4.59. Given $f \in C^{\infty}(B)$ and $t \in \mathbb{R}^{+}$, put

$$
\begin{aligned}
& \mathcal{W}_{+}\left(G_{i j}, A_{\alpha}^{i}, g_{\alpha \beta}, f, t\right) \\
& =\int_{B}\left[t \left(|\nabla f|^{2}+R-\frac{1}{4} g^{\alpha \beta} G^{i j} G_{j k, \alpha} G^{k l} G_{l i, \beta}\right.\right. \\
& \left.\left.\quad-\frac{1}{4} g^{\alpha \gamma} g^{\beta \delta} G_{i j} F_{\alpha \beta}^{i} F_{\gamma \delta}^{j}\right)-f+n\right](4 \pi t)^{-\frac{n}{2}} e^{-f} \mathrm{dvol}_{B} .
\end{aligned}
$$

If $N=0$, i.e., if $M=B$, then this is the same as the Feldman-Ilmanen-Ni $W_{+}$-functional [14].

In what follows, we will need a lower bound for $\mathcal{W}_{+}$in terms of the scalar curvature of $M$ and the volume of $B$.

Lemma 4.61. If $(4 \pi t)^{-\frac{n}{2}} \int_{B} e^{-f} \mathrm{dvol}_{B}=1$ then

$$
\mathcal{W}_{+}\left(G_{i j}, A_{\alpha}^{i}, g_{\alpha \beta}, f, t\right) \geq t \bar{R}_{\min }+n+\frac{n}{2} \ln (4 \pi)-\ln \left(t^{-\frac{n}{2}} \operatorname{vol}\left(B, g_{\alpha \beta}(t)\right)\right) \text {. }
$$


Proof. From (4.47),

$$
\begin{aligned}
& \mathcal{W}_{+}\left(G_{i j}, A_{\alpha}^{i}, g_{\alpha \beta}, f, t\right) \\
& =\int_{B}\left[t\left(\left|\nabla f+\nabla \ln \sqrt{\operatorname{det}\left(G_{i j}\right)}\right|^{2}+\bar{R}\right)-f+n\right](4 \pi t)^{-\frac{n}{2}} e^{-f} \mathrm{dvol}_{B} \\
& \geq t \bar{R}_{\min }+n-(4 \pi t)^{-\frac{n}{2}} \int_{B} f e^{-f} \operatorname{dvol}_{B} \\
& \geq t \bar{R}_{\min }+n+\frac{n}{2} \ln (4 \pi)-\ln \left(t^{-\frac{n}{2}} \operatorname{vol}\left(B, g_{\alpha \beta}(t)\right)\right),
\end{aligned}
$$

where we used Jensen's inequality. This proves the lemma.

The next proposition says that if $f$ satisfies a conjugate heat equation then $\mathcal{W}_{+}$is monotonic under the Ricci flow.

Proposition 4.64. Under the flow equations

$$
\begin{aligned}
\frac{\partial G_{i j}}{\partial t} & =g^{\alpha \beta} G_{i j ; \alpha \beta}-g^{\alpha \beta} G^{k l} G_{i k, \alpha} G_{l j, \beta}-\frac{1}{2} g^{\alpha \gamma} g^{\beta \delta} G_{i k} G_{j l} F_{\alpha \beta}^{k} F_{\gamma \delta}^{l}, \\
\frac{\partial A_{\alpha}^{i}}{\partial t} & =-g^{\gamma \delta} F_{\alpha \gamma ; \delta}^{i}-g^{\gamma \delta} G^{i j} G_{j k, \gamma} F_{\alpha \delta}^{k}, \\
\frac{\partial g_{\alpha \beta}}{\partial t}= & -2 R_{\alpha \beta}+\frac{1}{2} G^{i j} G_{j k, \alpha} G^{k l} G_{l i, \beta}+g^{\gamma \delta} G_{i j} F_{\alpha \gamma}^{i} F_{\beta \delta}^{j}, \\
\frac{\partial\left(e^{-f}\right)}{\partial t}= & -\nabla^{2} e^{-f}+\left(R-\frac{1}{4} g^{\alpha \beta} G^{i j} G_{j k, \alpha} G^{k l} G_{l i, \beta},\right. \\
& \left.\quad-\frac{1}{2} g^{\alpha \gamma} g^{\beta \delta} G_{i j} F_{\alpha \beta}^{i} F_{\gamma \delta}^{j}+\frac{n}{2 t}\right) e^{-f}
\end{aligned}
$$

one has

$$
\begin{aligned}
& \frac{d}{d t} \mathcal{W}_{+}\left(G_{i j}, A_{\alpha}^{i}, g_{\alpha \beta}, f, t\right) \\
& =\frac{t}{2} \int_{B} \mid g^{\alpha \beta} G_{i j ; \alpha \beta}-g^{\alpha \beta} G^{k l} G_{i k, \alpha} G_{l j, \beta} \\
& \quad-\frac{1}{2} g^{\alpha \gamma} g^{\beta \delta} G_{i k} G_{j l} F_{\alpha \beta}^{k} F_{\gamma \delta}^{l}-\left.g^{\alpha \beta} G_{i j, \alpha} f_{, \beta}\right|^{2}(4 \pi t)^{-\frac{n}{2}} e^{-f} \mathrm{dvol}_{B} \\
& \quad+t \int_{B}\left|g^{\gamma \delta} F_{\alpha \gamma ; \delta}^{i}+g^{\gamma \delta} G^{i j} G_{j k, \gamma} F_{\alpha \delta}^{k}-g^{\gamma \delta} f_{, \gamma} F_{\alpha \delta}^{k}\right|^{2}(4 \pi t)^{-\frac{n}{2}} e^{-f} \mathrm{dvol}_{B} \\
& +2 t \int_{B} \mid R_{\alpha \beta}-\frac{1}{4} G^{i j} G_{j k, \alpha} G^{k l} G_{l i, \beta} \\
& \quad-\frac{1}{2} g^{\gamma \delta} G_{i j} F_{\alpha \gamma}^{i} F_{\beta \delta}^{j}+f_{; \alpha \beta}+\left.\frac{1}{2 t} g_{\alpha \beta}\right|^{2}(4 \pi t)^{-\frac{n}{2}} e^{-f} \mathrm{dvol}_{B} \\
& \quad+\frac{1}{4} \int_{B} g^{\alpha \gamma} g^{\beta \delta} G_{i j} F_{\alpha \beta}^{i} F_{\gamma \delta}^{j}(4 \pi t)^{-\frac{n}{2}} e^{-f} \mathrm{dvol}_{B} .
\end{aligned}
$$


Proof. The proof is along the lines of the proof of Corollary 4.18.

We now look at what it means for $\mathcal{W}_{+}$to be constant along the flow (4.65).

Proposition 4.67. If $\mathcal{W}_{+}\left(G_{i j}, A_{\alpha}^{i}, g_{\alpha \beta}, f, t\right)$ is constant in then $F_{\alpha \beta}^{i}=0, \operatorname{det}\left(G_{i j}\right)$ is constant and

$$
\begin{aligned}
g^{\alpha \beta} G_{i j ; \alpha \beta}-g^{\alpha \beta} G^{k l} G_{i k, \alpha} G_{l j, \beta} & =0, \\
R_{\alpha \beta}-\frac{1}{4} G^{i j} G_{j k, \alpha} G^{k l} G_{l i, \beta}+\frac{1}{2 t} g_{\alpha \beta} & =0 .
\end{aligned}
$$

Proof. From (4.66), we see first that $F_{\alpha \beta}^{i}=0$. Then we also see that

$$
g^{\alpha \beta} G_{i j ; \alpha \beta}-g^{\alpha \beta} G^{k l} G_{i k, \alpha} G_{l j, \beta}-g^{\alpha \beta} G_{i j, \alpha} f_{, \beta}=0
$$

and

$$
R_{\alpha \beta}-\frac{1}{4} G^{i j} G_{j k, \alpha} G^{k l} G_{l i, \beta}+f_{; \alpha \beta}+\frac{1}{2 t} g_{\alpha \beta}=0 .
$$

As in the proof of Proposition 4.21, we can show from (4.69) that $\operatorname{det}\left(G_{i j}\right)$ is constant. Then equations (4.29) and (4.30) hold. From (4.70), we have

$$
\int_{B}\left(\bar{R}+\frac{n}{2 t}\right) \mathrm{dvol}_{B}=0 .
$$

As in the proof of Proposition 4.21, we have

$$
\frac{\partial \bar{R}}{\partial t}=\nabla^{2} \bar{R}+2\left|\bar{R}_{i j}\right|^{2}+2\left|\bar{R}_{\alpha \beta}\right|^{2} .
$$

From (4.65), (4.69) and (4.70), the flow equations are

$$
\begin{gathered}
\frac{\partial G_{i j}}{\partial t}=g^{\alpha \beta} G_{i j, \alpha} f_{, \beta}, \\
\frac{\partial g_{\alpha \beta}}{\partial t}=2 f_{; \alpha \beta}+\frac{1}{t} g_{\alpha \beta} .
\end{gathered}
$$

It follows that

$$
\frac{\partial \bar{R}}{\partial t}=\langle\nabla f, \nabla \bar{R}\rangle-\frac{\bar{R}}{t}
$$

Thus

$$
\nabla^{2} \bar{R}+2\left|\bar{R}_{i j}\right|^{2}+2\left|\bar{R}_{\alpha \beta}\right|^{2}+\frac{\bar{R}}{t}=\langle\nabla f, \nabla \bar{R}\rangle
$$

Then

$$
\begin{aligned}
& \nabla^{2}\left(\bar{R}+\frac{n}{2 t}\right)+2\left|\bar{R}_{i j}\right|^{2}+2\left|\bar{R}_{\alpha \beta}+\frac{1}{2 t} g_{\alpha \beta}\right|^{2}-\frac{1}{t}\left(\bar{R}+\frac{n}{2 t}\right) \\
& =\left\langle\nabla f, \nabla\left(\bar{R}+\frac{n}{2 t}\right)\right\rangle .
\end{aligned}
$$


From (4.71), either $\bar{R}+\frac{n}{2 t}=0$ or $\bar{R}_{\text {min }}+\frac{n}{2 t}<0$. If $\bar{R}_{\min }+\frac{n}{2 t}<0$ then we obtain a contradiction to the minimum principle, applied to (4.76). Thus $\bar{R}+\frac{n}{2 t}=0$. From (4.76), it follows that $\bar{R}_{i j}=\bar{R}_{\alpha \beta}+\frac{1}{2 t} g_{\alpha \beta}=0$. This proves the proposition.

Lemma 4.77. Under the conclusion of Proposition 4.67, $G_{i j}$ and $A_{\alpha}^{i}$ are timeindependent, and $g_{\alpha \beta}$ is proportionate to $t$.

Proof. This follows from (4.65) and (4.68).

Remark 4.78. Equations (4.68) were called the harmonic-Einstein equations in [38], where they were used as an ansatz to construct expanding soliton solutions on the total spaces of flat vector bundles.

We now use $W_{+}$to analyze blowdown limits.

Proposition 4.79. Suppose that $(M, \bar{g}(\cdot))$ is a locally $\mathcal{G}$-invariant Ricci flow defined for all $t \in(0, \infty)$. Let $\left\{s_{i}\right\}_{i=1}^{\infty}$ be a sequence of positive numbers tending to infinity. Put $\bar{g}_{i}(t)=s_{i}^{-1} \bar{g}\left(s_{i} t\right)$. Suppose that $\lim _{i \rightarrow \infty} \bar{g}_{i}(\cdot)$ exists and equals $\bar{g}_{\infty}(\cdot)$ in the sense of Section 4.1, for a locally $\mathscr{E}$-invariant Ricci flow $\bar{g}_{\infty}(\cdot)$ with a compact base $B_{\infty}$, defined for $t \in(0, \infty)$. Writing $\bar{g}_{\infty}(\cdot) \equiv\left(G_{i j, \infty}(\cdot), A_{\alpha, \infty}^{i}(\cdot), g_{\alpha \beta, \infty}(\cdot)\right)$, we conclude that

1. $F_{\alpha \beta, \infty}^{i}=0$;

2. $\operatorname{det}\left(G_{i j, \infty}\right)$ is constant;

3. equations (4.68) are satisfied for $G_{i j, \infty}(\cdot)$ and $g_{\alpha \beta, \infty}(\cdot)$.

Proof. The proof is along the lines of the proof of Proposition 4.39.

We now look at some special solutions of (4.68). Recall that $\rho: \pi_{1}(B, b) \rightarrow$ $\mathrm{SL}(N)$ is the holonomy representation.

Proposition 4.80. Under the assumptions of Proposition 4.79, if $N=0$ then $\bar{g}_{\infty}(t)=t g_{\text {Ein }}$, where $g_{\text {Ein }}$ is an Einstein metric on $M=B$ with Einstein constant $-\frac{1}{2}$. If $N=1$ then $\bar{g}_{\infty}(t)$ is locally an isometric product of $\mathbb{R}$ or $S^{1}$ with $\left(B, t g_{\text {Ein }}\right)$, where $g_{\text {Ein }}$ is an Einstein metric on $B$ with Einstein constant $-\frac{1}{2}$. For any $N$, if $\operatorname{dim}(B)=1$ then with an appropriate choice of section $s$, we can locally write $G_{i j}(b)=\left(e^{b X}\right)_{i j}$ and $g_{B}=\frac{t}{2} \operatorname{Tr}\left(X^{2}\right) d b^{2}$, where $X$ is a real diagonal $(N \times N)$-matrix with vanishing trace.

If $\operatorname{dim}(B)=2$ and $N=2$ then $B$ has negative Euler characteristic. Also,

1. $\bar{g}$ is a locally product metric and $B$ has sectional curvature $-\frac{1}{2 t}$, or

2. $\rho$ fixes no point of the boundary of $\operatorname{SL}(2, \mathbb{R}) / \mathrm{SO}(2)=H^{2}$ and with the right choice of orientation of $\widetilde{B}$, the map $\widetilde{G}: \widetilde{B} \rightarrow H^{2}$ is holomorphic. 
Proof. The $N=0$ case is clear. As $\operatorname{det}\left(G_{i j}\right)$ is constant, if $N=1$ then we are in a local product situation. For any $N$, if $\operatorname{dim}(B)=1$ then the map $b \rightarrow G_{i j}(b)$ describes a geodesic in $\operatorname{SL}(N, \mathbb{R}) / \mathrm{SO}(N, \mathbb{R})$, from which the proposition follows. (See [38, Example 4.27]).

If $\operatorname{dim}(B)=2$ and $N=2$ then we can consider $\tilde{g}$ to be a $\rho$-equivariant harmonic map $u: \widetilde{B} \rightarrow H^{2}$. Choosing an orientation of $\widetilde{B}$, we use a local complex coordinate $z$ on $\widetilde{B}$. There is a solution to the first equation in (4.68) if and only if the representation $\rho: \pi_{1}(B) \rightarrow \operatorname{SL}(2, \mathbb{R})$ is not conjugate to a (nondiagonal) representation by upper triangular matrices [30], [36]. If there is a solution to the first equation in (4.68) then looking at the $d z^{2}$-component of the second equation in (4.68) gives

$$
u_{z} \overline{u_{\bar{z}}}=0 .
$$

We consider the subset of $\partial H^{2}$, the boundary at infinity of $H^{2}$, which is pointwise fixed by $\operatorname{Im}(\rho)$. It is either all of $\partial H^{2}$, two points in $\partial H^{2}$, one point in $\partial H^{2}$ or the empty set. If all of $\partial H^{2}$ is fixed by $\operatorname{Im}(\rho)$ then $\rho$ is the identity representation, $u$ descends to a harmonic function on $B$ (which must be constant) and $B$ has constant sectional curvature $-\frac{1}{2 t}$. If $\operatorname{Im}(\rho)$ fixes exactly two points of $\partial H^{2}$ then $\rho$ is conjugate to a diagonal representation and $u$ maps to a nontrivial geodesic in $H^{2}$. We can assume that $u$ is real-valued. Then equation (4.81) implies that $u$ is constant, which is a contradiction. As has been said, there is no solution to the first equation in (4.68) if $\operatorname{Im}(\rho)$ fixes a single point of $\partial H^{2}$. Finally, suppose that $\operatorname{Im}(\rho)$ fixes no point of $\partial H^{2}$. Then $u$ is constant or $d u$ has generic rank two. If $u$ is constant then $\bar{g}$ is a locally product metric. Suppose that $u$ is nonconstant. As $d u$ has generic rank 2, equation (4.81) implies that $u$ is holomorphic or antiholomorphic. If $u$ is antiholomorphic then we change the orientation of $\widetilde{B}$ to make $u$ holomorphic. As $u$ is nonconstant, Liouville's theorem implies that $B$ has negative Euler characteristic.

Remark 4.82. The solutions with $\operatorname{dim}(B)=1, G_{i j}(b)=\left(e^{b X}\right)_{i j}$ and $g_{B}=$ $\frac{t}{2} \operatorname{Tr}\left(X^{2}\right) d b^{2}$ are generalized Sol-solutions.

Remark 4.83. When $\operatorname{dim}(B)=2$ and $N=2$, the equations (4.68) arose independently in the paper [52] on Kähler-Ricci flow. In that paper, which is in the holomorphic setting, the map $G$ arises as the classifying map for the torus bundle of an elliptic fibration. The term $\frac{1}{4} G^{i j} G_{j k, \alpha} G^{k l} G_{l i, \beta}$ of (4.68) is called the WeilPetersson term. The second equation of (4.68), in the Kähler case, is considered to be a generalized Kähler-Einstein equation for the geometry of a collapsing limit.

Remark 4.84. All of the results of this section extend to the case when $B$ is an orbifold, $E$ is a flat orbifold $\mathscr{E}$-bundle over $B$, a manifold $M$ is the total space of an orbifold fiber bundle $\pi: M \rightarrow B$ and $\mathscr{G}$ acts locally freely on $M$ (via a map $E \times{ }_{B} M \rightarrow M$ ) with orbifold quotient $B$. 


\section{Equivalence classes of étale groupoids}

Let $\&$ be a complete effective path-connected Hausdorff étale groupoid that admits an invariant Riemannian metric on the space of units $G^{(0)}$. We assume that

1. Go equals its closure $\bar{G}$;

2. the local symmetry sheaf $g$ of $\&$ is a locally constant sheaf of abelian Lie algebras isomorphic to $\mathbb{R}^{N}$.

Example 5.1. Let $M$ be the total space of a twisted abelian principal $\mathscr{H}$-bundle as in Section 4.1. We can take $G=E \times_{B} M$, where the flat bundle $E$ has the étale topology, with $\Im^{(0)}=M$. The local symmetry sheaf comes from the flat vector bundle $\pi^{*} e$ on $M$.

We can perform a similar construction in the setting of Remark 4.84, where $M$ is a manifold and $B$ is an orbifold.

The results of Section 4 extend to the setting of a Ricci flow on $G$, under the analogous curvature and diameter assumptions, provided that $G$ is locally free. The reason is that the local structure of such an étale groupoid is the same as the local structure considered in Section 4 [22, Corollary 3.2.2]. We can then perform the integrals of Section 4 over the orbit space of $\$ 5$ and derive the same consequences as in Section 4.

It will be useful to determine the global structure of such étale groupoids, at least in low dimensions.

Proposition 5.2. Suppose that $\mathbb{S}$ is locally free. Then the orbit space $\mathcal{O}$ is an orbifold. There is a flat (orbifold) $\mathbb{R}^{N}$-bundle e on $\mathcal{O}$ associated to $\mathbb{6}$.

If $\operatorname{dim}(\mathcal{O})=1$ then $\mathbb{G}$ is classified by the isomorphism class of $e$.

In general, if $e$ is trivial then (S) is equivalent to the groupoid of a principal bundle over $\mathcal{O}$. It is classified up to groupoid equivalence by the orbits of $\operatorname{GL}(N, \mathbb{R})$ on $\mathrm{H}^{2}\left(\mathcal{O} ; \mathbb{R}^{N}\right)$.

Proof. The proof is similar to the classification in [23] of the transverse structure of Riemannian foliations with low-codimension leaves. (As the paper [23] considers Riemannian groupoids that may not equal their closure, there is an additional step in [23] which consists of analyzing the restriction of the groupoid to an orbit closure. Since we only deal with étale groupoids that equal their closures, we do not have to deal with this complication.)

Given $x \in G^{(0)}$, let $\mathcal{O}_{x}$ be its orbit. There is an invariant neighborhood of the orbit whose groupoid structure is described by [22, Corollary 3.2.2]. In particular, the point in the orbit space $\mathcal{O}$, corresponding to $\mathcal{O}_{x}$, has a neighborhood $U$ that is 
homeomorphic to $V / \varsigma_{x}^{x}$, where $V$ is a representation space for the isotropy group $\varsigma_{x}^{x}$. This gives the orbifold structure on the orbit space.

The classification of such étale groupoids comes from the bundle theory developed in [22, Section 2.3], which we now follow. For notation, if $G$ is a topological group then let $G_{\delta}$ denote $G$ with the discrete topology. Suppose first that the isotropy groups $\varsigma_{x}^{x}$ are trivial, so the orbifold $\mathcal{O}$ is a manifold. Let $U \subset \mathcal{O}$ be a neighborhood of $\mathcal{O}_{x}$ as above. Let $\pi: \mathscr{G}^{(0)} \rightarrow \mathcal{O}$ be the quotient map. By [22, Corollary 3.2.2], the restriction of $\left(5\right.$ to $\pi^{-1}(U)$ is equivalent to the cross-product groupoid $\left(\mathbb{R}^{N} \times U\right) \rtimes \mathbb{R}_{\delta}^{N}$, where $\mathbb{R}_{\delta}^{N}$ acts on $\mathbb{R}^{N}$ by translation and acts trivially on $U$. This gives the local structure of $\$$. It remains to determine the possible ways to glue these local structures together.

To follow the notation of [22, Section 2.1], put $\Gamma=\mathbb{R}_{\delta}^{N} \subset \operatorname{Diff}\left(\mathbb{R}^{N}\right)_{\delta}$. The normalizer $N^{\Gamma}$ of $\Gamma$ in $\operatorname{Diff}\left(\mathbb{R}^{N}\right)$ is $\mathbb{R}^{N} \tilde{\times} \operatorname{GL}(N, \mathbb{R})$ and the centralizer is $C^{\Gamma}=\mathbb{R}^{N}$. We give $N^{\Gamma}$ the topology $\mathbb{R}^{N} \tilde{\times} \operatorname{GL}(N, \mathbb{R})_{\delta}$.

Following the discussion in [22, Section 2.1], suppose that $U \subset \mathcal{O}$ is an open set. Consider the cross-product groupoid $\left(\mathbb{R}^{N} \times U\right) \rtimes \mathbb{R}_{\delta}^{N}$. Let $\mathcal{E}(U)$ be the selfequivalences of $\left(\mathbb{R}^{N} \times U\right) \rtimes \mathbb{R}_{\delta}^{N}$ that project onto the identity of $U$. This forms a sheaf $\mathcal{E}$ on $\mathcal{O}$. We can cover $\mathcal{O}$ by open sets $U$ such that $\pi^{-1}(U)$ is equivalent to $\left(\mathbb{R}^{N} \times U\right) \rtimes \mathbb{R}_{\delta}^{N}$. It follows that the étale groupoids in question are classified by the set $\mathrm{H}^{1}(\mathcal{O} ; \underline{\mathcal{E}})$ [22, Proposition 2.3.2].

To compute $\mathrm{H}^{1}(\mathcal{O} ; \underline{\mathcal{E}})$, let $\underline{\mathbb{R}}^{N}$ be the sheaf on $\mathcal{O}$ for which $\underline{\mathbb{R}}^{N}(U)$ consists of

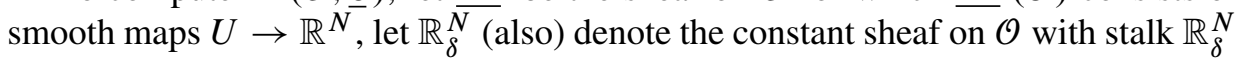
and let $\operatorname{GL}(N, \mathbb{R})_{\delta}$ (also) denote the constant sheaf on $\mathcal{O}$ with stalk $\operatorname{GL}(N, \mathbb{R})_{\delta}$. As in $[22,(2.4 .2)]$ there is a short exact sequence of sheaves

$$
0 \longrightarrow \underline{\mathbb{R}^{N}} / \mathbb{R}_{\delta}^{N} \longrightarrow \underline{\mathcal{E}} \longrightarrow \operatorname{GL}(N, \mathbb{R})_{\delta} \longrightarrow 0 .
$$

From [15, Théorème 1.2], this short exact sequence of sheaves gives rise to an exact sequence of pointed sets

$$
\begin{aligned}
\cdots \longrightarrow \mathrm{H}^{0}\left(\mathcal{O} ; \mathrm{GL}(N, \mathbb{R})_{\delta}\right) \longrightarrow & \mathrm{H}^{1}\left(\mathcal{O} ; \underline{\mathbb{R}^{N}} / \mathbb{R}_{\delta}^{N}\right) \\
& \longrightarrow \mathrm{H}^{1}(\mathcal{O} ; \underline{\mathcal{E}}) \longrightarrow \mathrm{H}^{1}\left(\mathcal{O} ; \mathrm{GL}(N, \mathbb{R})_{\delta}\right) .
\end{aligned}
$$

The set $\mathrm{H}^{1}\left(\mathcal{O} ; \mathrm{GL}(N, \mathbb{R})_{\delta}\right)$ is the same as the set of homomorphisms $\pi_{1}(\mathcal{O}) \rightarrow$ $\operatorname{GL}(N, \mathbb{R})$ modulo conjugation by elements of $\operatorname{GL}(N, \mathbb{R})$ or, equivalently, the set of equivalence classes of flat $\mathbb{R}^{N}$-vector bundles on $\mathcal{O}$. The image of the classifying element of $\left(5\right.$, under the map $\mathrm{H}^{1}(\mathcal{O} ; \underline{\mathcal{E}}) \longrightarrow \mathrm{H}^{1}\left(\mathcal{O} ; \mathrm{GL}(N, \mathbb{R})_{\delta}\right)$, classifies the flat $\mathbb{R}^{N}$-vector bundle $e$ mentioned in Proposition 5.2. More explicitly, the transition functions of $e$ come from the image under $\underline{\mathcal{E}} \longrightarrow \operatorname{GL}(N, \mathbb{R})_{\delta}$ of the transition functions of $\$ s$.

The short exact sequence

$$
0 \longrightarrow \mathbb{R}_{\delta}^{N} \longrightarrow \underline{\mathbb{R}^{N}} \longrightarrow \underline{\mathbb{R}^{N}} / \mathbb{R}_{\delta}^{N} \longrightarrow 0
$$


of sheaves of abelian groups gives a long exact sequence

$$
\begin{aligned}
\cdots \longrightarrow \mathrm{H}^{1}\left(\mathcal{O} ; \underline{\mathbb{R}^{N}}\right) \longrightarrow & \mathrm{H}^{1}\left(\mathcal{O} ; \underline{\mathbb{R}^{N}} / \mathbb{R}_{\delta}^{N}\right) \\
& \longrightarrow \mathrm{H}^{2}\left(\mathcal{O} ; \mathbb{R}_{\delta}^{N}\right) \longrightarrow \mathrm{H}^{2}\left(\mathcal{O} ; \underline{\mathbb{R}^{N}}\right) \longrightarrow \cdots
\end{aligned}
$$

of abelian groups. As $\underline{\mathbb{R}^{N}}$ is a fine sheaf, it follows from (5.6) that $\mathrm{H}^{1}\left(\mathcal{O} ; \underline{\mathbb{R}^{N}} / \mathbb{R}_{\delta}^{N}\right) \cong$ $\mathrm{H}^{2}\left(\mathcal{O} ; \mathbb{R}_{\delta}^{N}\right)=\mathrm{H}^{2}\left(\mathcal{O} ; \mathbb{R}^{N}\right)$.

As $\mathrm{H}^{0}$ consists of global sections, (5.4) gives an exact sequence of pointed sets

$$
\mathrm{GL}(N, \mathbb{R}) \longrightarrow \mathrm{H}^{2}\left(\mathcal{O} ; \mathbb{R}^{N}\right) \longrightarrow \mathrm{H}^{1}(\mathcal{O} ; \underline{\mathcal{E}}) \longrightarrow \mathrm{H}^{1}\left(\mathcal{O} ; \mathrm{GL}(N, \mathbb{R})_{\delta}\right)
$$

If $\operatorname{dim}(\mathcal{O})=1$ then from $(5.7)$, the map $\mathrm{H}^{1}(\mathcal{O} ; \underline{\mathcal{E}}) \longrightarrow \mathrm{H}^{1}\left(\mathcal{O} ; \operatorname{GL}(N, \mathbb{R})_{\delta}\right)$ is injective. Thus $\&$ is determined up to groupoid equivalence by the isomorphism class of the flat vector bundle $e$.

If $\mathcal{O}$ has arbitrary dimension, suppose that $e$ is trivial. Consider the preimage under $\mathrm{H}^{1}(\mathcal{O} ; \underline{\mathcal{E}}) \longrightarrow \mathrm{H}^{1}\left(\mathcal{O} ; \operatorname{GL}(N, \mathbb{R})_{\delta}\right)$ of the element in $\mathrm{H}^{1}\left(\mathcal{O} ; \mathrm{GL}(N, \mathbb{R})_{\delta}\right)$ corresponding to the identity representation. By (5.7), this preimage can be identified with the orbit space for the action of $\operatorname{GL}(N, \mathbb{R})$ on $\mathrm{H}^{2}\left(\mathcal{O} ; \mathbb{R}^{N}\right)$. Any such orbit contains an element of $\operatorname{Im}\left(\mathrm{H}^{2}\left(\mathcal{O} ; \mathbb{Z}^{N}\right) \rightarrow \mathrm{H}^{2}\left(\mathcal{O} ; \mathbb{R}^{N}\right)\right)$, which implies that $\circlearrowleft$ is equivalent to the étale groupoid arising from some principal $T^{N}$-bundle on $\mathcal{O}$.

The preceding considerations extend to the case when the (finite) isotropy groups $\mathcal{S}_{x}^{x}$ are not all trivial. In that case, $\mathcal{O}$ is an orbifold and the argument extends to the orbifold setting. For example, $\mathrm{H}^{*}\left(\mathcal{O} ; \mathbb{R}^{N}\right)$ has to be interpreted as an orbifold cohomology group.

Remark 5.8. If one starts with an (untwisted) principal $\mathcal{E}$-bundle, with $\mathcal{E}$ abelian, then the triviality of the corresponding étale groupoid is determined by whether or not $\left\{\int_{B} F^{i}\right\}_{i=1}^{N}$ vanishes in $\mathrm{H}^{2}\left(B ; \mathbb{R}^{N}\right)$.

Suppose that the étale groupoid is nontrivial and $\left\{\bar{g}_{j}\right\}_{j=1}^{\infty}$ is a sequence of invariant metrics on the principal $\mathscr{E}$-bundle, so that there is a limiting invariant metric $\bar{g}_{\infty}$. It is possible that the curvatures $\left\{F^{i}\right\}_{i=1}^{N}$ approach zero in norm as $j \rightarrow \infty$. If this is the case then $\bar{g}_{\infty}$ will live on a distinct étale groupoid, as its curvature $\left\{F^{i}\right\}_{i=1}^{N}$ vanishes. This phenomenon occurs in the rescaled Ricci flow on the unit circle bundle of a surface of constant negative curvature.

On the other hand, if we start with a trivial étale groupoid and $\left\{\bar{g}_{j}\right\}_{j=1}^{\infty}$ is a noncollapsing sequence of invariant metrics on the principal $\mathcal{E}$-bundle then any limiting invariant metric $\bar{g}_{\infty}$ will necessarily be on the same étale groupoid.

The relevance of Proposition 5.2 is that for étale groupoids which satisfy its hypotheses, we can discuss convergence of Ricci flow solutions on such étale groupoids in terms of convergence of invariant Ricci flow solutions on twisted principal bundles. 
Example 5.9. Suppose that $M$ is the total space of a principal $S^{1}$-bundle over a compact oriented surface $B$. Given a subgroup $\mathbb{Z}_{k} \subset S^{1}$, let $M / \mathbb{Z}_{k}$ be the quotient space. It is also the total space of a principal $S^{1}$-bundle over $B$.

The (discrete) $S^{1}$-action on a principal $S^{1}$-bundle gives an étale groupoid. The map $M \rightarrow M / \mathbb{Z}_{k}$ gives an equivalence of étale groupoids, in the sense of [2, Chapter III.E.2.4]. However, the Euler class of the circle bundle $M / \mathbb{Z}_{k} \rightarrow B$ is $k$ times that of the circle bundle $M \rightarrow B$. This shows that the Euler class of the circle bundle is not an invariant of the groupoid equivalence class. Instead, all that is relevant is whether or not the rational Euler class vanishes.

Example 5.10. If $\operatorname{dim}(\mathcal{O})=1$ then any homomorphism $\alpha: \pi_{1}(\mathcal{O}) \rightarrow \operatorname{GL}(N, \mathbb{R})$ gives rise to an étale groupoid with unit space $\mathscr{G}^{(0)}=\mathbb{R}^{N} \times_{\alpha} \widetilde{\mathcal{O}}$.

If $\mathcal{O}$ is a closed orientable 2-dimensional orbifold then $\mathrm{H}^{2}\left(\mathcal{O} ; \mathbb{R}^{N}\right) \cong \mathbb{R}^{N}$ and the action of $\operatorname{GL}(N, \mathbb{R})$ on $\mathrm{H}^{2}\left(\mathcal{O} ; \mathbb{R}^{N}\right)$ has two orbits, namely the zero element and the nonzero elements. Thus if $e$ is trivial then there are two equivalence classes of such groupoids with orbit space $\mathcal{O}$, one corresponding to a vanishing "Euler class" and one corresponding to a nonvanishing "Euler class".

Suppose that $M$ is the total space of a twisted principal $\mathbb{R}^{N}$-bundle. Let $\bar{g}$ be an invariant metric on $M$. We recall that there are two distinct connections in this situation, the flat connection on the twisting bundle $\mathcal{E}$ and the connection $A$ on the twisted principal bundle. We will use the following lemma later.

Lemma 5.11. Let $\pi: M \rightarrow B$ be a twisted principal $\mathbb{R}^{N}$-bundle. Given $G_{i j}$ and $g_{\alpha \beta}$, let $A_{1}$ and $A_{2}$ be two flat connections on $M$. Let $\bar{g}_{1}$ and $\bar{g}_{2}$ be the corresponding invariant metrics on $M$. Then their underlying Riemannian groupoids are equivalent.

Proof. Let $\left\{U_{i}\right\}$ be a covering of $B$ by open contractible sets. Let $U=\left\{\pi^{-1}\left(U_{i}\right)\right\}$ be the corresponding covering of $M$ and let $\mathbb{F} u$ be the localization of $G 5$ [38, Section 5.2]. In our case, elements of $\sigma u$ are quadruples $\left(i, p_{i}, p_{j}, j\right)$ with $p_{i} \in \pi^{-1}\left(U_{i}\right), p_{j} \in$ $\pi^{-1}\left(U_{j}\right)$ and $\pi\left(p_{i}\right)=\pi\left(p_{j}\right)$. The multiplication is $\left(i, p_{i}, p_{j}, j\right) \cdot\left(j, p_{j}, p_{k}, k\right)=$ $\left(i, p_{i}, p_{k}, k\right)$. The units $\sigma_{u}^{(0)}$ are quadruples $\left(i, p_{i}, p_{i}, i\right)$ and the source and range maps are $s\left(i, p_{i}, p_{j}, j\right)=\left(j, p_{j}, p_{j}, j\right)$ and $r\left(i, p_{i}, p_{j}, j\right)=\left(i, p_{i}, p_{i}, i\right)$. Let $s_{i}^{1}: U_{i} \rightarrow \pi^{-1}\left(U_{i}\right)$ be a section for which $\left(s_{i}^{1}\right)^{*} A_{1}=0$. Similarly, let $s_{i}^{2}: U_{i} \rightarrow$ $\pi^{-1}\left(U_{i}\right)$ be a section for which $\left(s_{i}^{2}\right)^{*} A_{2}=0$. Define a map $F: \mathbb{G} u \rightarrow \mathbb{G} u$ by $F\left(i, p_{i}, p_{j}, j\right)=\left(i, p_{i}+s_{i}^{2}\left(u_{i}\right)-s_{i}^{1}\left(u_{i}\right), p_{j}+s_{j}^{2}\left(u_{j}\right)-s_{j}^{1}\left(u_{j}\right), j\right)$, where $u_{i}=\pi\left(p_{i}\right), u_{j}=\pi\left(p_{j}\right)$ and we write the action of $\mathbb{R}^{N}$ additively. Then $F$ is a groupoid isomorphism. On the space of units, $F\left(i, p_{i}, p_{i}, i\right)=\left(i, p_{i}+s_{i}^{2}\left(u_{i}\right)-\right.$ $\left.s_{i}^{1}\left(u_{i}\right), p_{i}+s_{i}^{2}\left(u_{i}\right)-s_{i}^{1}\left(u_{i}\right), i\right)$ and so $F$ sends the section $s_{i}^{1}$ to $s_{i}^{2}$. It follows that $F$ is an isomorphism of Riemannian groupoids. 


\section{Convergence arguments and universal covers}

In this section we prove Theorem 1.2. In Section 6.1 we prove convergence to a locally homogeneous Ricci flow on an étale groupoid. In Section 6.2 we promote this to convergence on the universal cover of $M$.

6.1. Convergence arguments. In this subsection we show that under the hypotheses of Theorem 1.2, there is a rescaling limit which is a locally homogeneous expanding soliton solution on an étale groupoid. To do this, if $\overline{\mathcal{O}}$ is the closure of the orbit of $g(\cdot)$ under the action of the parabolic rescaling semigroup $\mathbb{R}^{\geq 1}$ then we define a stratification of $\overline{\mathcal{O}}$ in terms of the number of local symmetries. We let $k_{0}$ be the maximal number of local symmetries that can occur in a rescaling limit of $g(\cdot)$. This corresponds to a maximally collapsed limit. The first step is to show that $k_{0}$ determines the Thurston type of $M$, and that there is a sequence of rescalings of $g(\cdot)$ which approaches the corresponding locally homogeneous expanding soliton.

In order to show that any rescaling limit $\bar{g}(\cdot)$ is a locally homogeneous expanding soliton (except possibly in the $\widetilde{\mathrm{SL}_{2}(\mathbb{R})}$ case), we use further arguments. We show that any rescaling limit has $k_{0}$ local symmetries. We then use a compactness argument, along with the local stability of the space of expanders, to show that $\bar{g}(\cdot)$ is a locally homogeneous expanding soliton.

So assume that $g(\cdot)$ is a Ricci flow solution on a connected closed 3-manifold $M$, defined for $t \in(1, \infty)$, with $\sup _{t \in(1, \infty)} t\|\operatorname{Riem}(g(t))\|_{\infty} \leq K<\infty$ and $\sup _{t \in(1, \infty)} t^{-\frac{1}{2}} \operatorname{diam}(g(t)) \leq D<\infty$. From Proposition 3.5, $M$ has a single geometric piece. Given $s \in[1, \infty)$, put $g_{s}(t)=\frac{1}{s} g(s t)$. Then for all $s$, we have $\sup _{t \in(1, \infty)} t\left\|\operatorname{Riem}\left(g_{s}(t)\right)\right\|_{\infty} \leq K$ and $\sup _{t \in(1, \infty)} t^{-\frac{1}{2}} \operatorname{diam}\left(g_{s}(t)\right) \leq D$. By Proposition 3.2, the family of Ricci flow solutions $\left\{g_{s}(\cdot)\right\}_{s \in[1, \infty)}$ is sequentially precompact among Ricci flow solutions on étale groupoids.

Let $\overline{\mathcal{O}}$ be the sequential closure of the forward orbit $\left\{g_{s}(\cdot)\right\}_{s \in[1, \infty)}$. Let $\overline{\mathcal{O}}_{(k)}$ be the elements of $\overline{\mathcal{O}}$ with a $k$-dimensional local symmetry sheaf $\underline{\mathrm{g}}$.

Lemma 6.1. If $\hat{g}(\cdot) \in \overline{\mathcal{O}}$ then the underlying étale groupoid of $\hat{g}(\cdot)$ is locally free.

Proof. If $\hat{g}(\cdot) \in \overline{\mathcal{O}}_{(0)}$ then there is nothing to show. If $\hat{g}(\cdot) \in \overline{\mathcal{O}}_{(1)}$ then the lemma follows from the fact there is no point $x \in \varsigma^{(0)}$ where the local Killing vector fields vanish simultaneously.

Suppose that $\hat{g}(\cdot) \in \overline{\mathcal{O}}_{(2)}$. Write $\hat{g}(\cdot)=\lim _{i \rightarrow \infty}\left(M, g_{s_{j}^{\prime}}(\cdot)\right)$ for some sequence $\left\{s_{j}^{\prime}\right\}_{j=1}^{\infty}$ tending to infinity. By [3], for any $\epsilon>0$, there is an integer $J_{\epsilon}<\infty$ so that if $j \geq J_{\epsilon}$ then there is a locally $T^{2}$-invariant Riemannian metric $g_{j}^{\prime}$ on $M$ which is $\epsilon$-close in the $C^{1}$-topology to $\frac{1}{s_{j}^{\prime}} g\left(s_{j}^{\prime}\right)$. Furthermore, one can take the sectional curvature of $g_{j}^{\prime}$ to be uniformly bounded in $\epsilon[48$, Theorem 2.1]. The collapsing is 
along the $T^{2}$ fibers. Taking a sequence of values of $\epsilon$ going to zero and choosing $j \geq J_{\epsilon}$, after passing to a subsequence we can say that $\hat{g}(1)=\lim _{j \rightarrow \infty}\left(M, g_{j}^{\prime}\right)$.

Let $S$ be the orbit space of the étale groupoid. It is a circle or an interval. If $S$ is a circle then $M$ is the total space of a $T^{2}$-bundle over $S^{1}$. (The fibers cannot be Klein bottles since $M$ is orientable.) Hence the local $T^{2}$-action on $\left(M, g_{j}^{\prime}\right)$ is free. Let $H \in \mathrm{SL}(2, \mathbb{Z})$ be the holonomy of the $T^{2}$-bundle, defined up to conjugation in $\operatorname{SL}(2, \mathbb{Z})$. Given $M$, there is a finite number of possibilities for $H$, as follows from [50, pp. 439, 469-470, 481-482]. After passing to a subsequence, we can assume that there is a single such $H$. For each $j$, the $T^{2}$-bundle with invariant metric $g_{j}^{\prime}$ is the total space of a twisted principal $T^{2}$-bundle over $S^{1}$, where the twisting bundle $E$ is a flat $T^{2}$-bundle on $S^{1}$ with holonomy $H$. From Proposition 5.2, for all $j$ these give rise to equivalent étale groupoids. Looking at how one constructs the limiting Riemannian groupoid as $j \rightarrow \infty$ [38, Proposition 5.9], it follows that $\hat{g}(\cdot)$ is defined on this same étale groupoid. In particular, it is locally free.

If $S$ is an interval then as in the proof of Proposition 3.5, the asphericity of $M$ implies that the local $T^{2}$-action on $M$ is locally free. Then $M$ is the total space of an orbifold $T^{2}$-bundle over the orbifold $S$. As $S$ is double covered by a circle, we can take a double cover $\widehat{M}$ of $M$ which is the total space of a $T^{2}$-bundle over $S^{1}$. Applying the preceding argument $\mathbb{Z}_{2}$-equivariantly to $\widehat{M}$, we conclude that the underlying étale groupoid of $\hat{g}(\cdot)$ is again locally free.

Finally, suppose that $\hat{g}(\cdot) \in \overline{\mathcal{O}}_{(3)}$. Write $\hat{g}(\cdot)=\lim _{i \rightarrow \infty}\left(M, g_{s_{j}^{\prime}}(\cdot)\right)$ for some sequence $\left\{s_{j}^{\prime}\right\}_{j=1}^{\infty}$ tending to infinity. Then the orbit space $S$ of the étale groupoid is a point and $\left\{\left(M, \frac{1}{s_{j}^{\prime}} g\left(s_{j}^{\prime}\right)\right)\right\}_{j=1}^{\infty}$ Gromov-Hausdorff converges, with bounded sectional curvature, to a point. That is, $M$ is almost flat and so is an infranilmanifold [19]. There is a finite normal cover $M_{0}$ of $M$ which is diffeomorphic to a flat manifold or a nilmanifold. Let $g_{0}(\cdot)$ be the lift of $g(\cdot)$ to $M_{0}$ and let $\hat{g}_{0}(\cdot)$ be the corresponding limiting Ricci flow on an étale groupoid, with $\hat{g}(\cdot)$ as a finite quotient. By [3], for any $\epsilon>0$, there is an integer $J_{\epsilon}<\infty$ so that if $j \geq J_{\epsilon}$ then there is a left-invariant Riemannian metric $g_{j}^{\prime}$ on $M_{0}$, of $\mathbb{R}^{3}$ or Nil-type, which is $\epsilon$-close in the $C^{1}$-topology to $\frac{1}{s_{j}^{\prime}} g_{0}\left(s_{j}^{\prime}\right)$. Furthermore, one can take the sectional curvature of $g_{j}^{\prime}$ to be uniformly bounded in $\epsilon$ [48, Theorem 2.1]. The collapsing is along all of $M_{0}$. Taking a sequence of values of $\epsilon$ going to zero and choosing $j \geq J_{\epsilon}$, after passing to a subsequence we can say that $\hat{g}_{0}(1)=\lim _{j \rightarrow \infty}\left(M_{0}, g_{j}^{\prime}\right)$. Looking at how one constructs the limiting Riemannian groupoid as $j \rightarrow \infty$ [38, Proposition 5.9], it follows that the underlying étale groupoid of $\hat{g}_{0}(1)$ is a cross-product groupoid $\mathbb{R}^{3} \rtimes \mathbb{R}_{\delta}^{3}$ or $\mathrm{Nil} \rtimes \mathrm{Nil}_{\delta}$, where $\delta$ denotes the discrete topology. Hence the underlying étale groupoid of $\hat{g}(\cdot)$ is locally free.

The relevance of Proposition 6.1 is that it allows us to use Proposition 4.79 to analyze blowdown limits of $\hat{g}(\cdot)$. 
Let $k_{0}$ be the largest $k$ so that $\overline{\mathcal{O}}_{(k)}$ is nonempty. For simplicity of terminology, we will say that a Ricci flow on an étale groupoid is a locally homogeneous expanding soliton if there is some homogeneous expanding soliton to which the Ricci flow on the unit space of the étale groupoid is locally isometric.

Proposition 6.2. If $k_{0}=0$ then $M$ admits an $H^{3}$-structure. If $k_{0}=1$ then $M$ admits an $H^{2} \times \mathbb{R}$ or $\widetilde{\mathrm{SL}_{2}(\mathbb{R})}$-structure. If $k_{0}=2$ then $M$ admits a Sol-structure. If $k_{0}=3$ then $M$ admits an $\mathbb{R}^{3}$ or Nil-structure.

In all of these cases, there is a sequence $\left\{s_{j}\right\}_{j=1}^{\infty}$ tending to infinity so that $\lim _{j \rightarrow \infty}\left(M, g_{s_{j}}(\cdot)\right)$ exists as a Ricci flow solution on an étale groupoid, and is a locally homogeneous expanding soliton of type

- $H^{3}$ if $k_{0}=0$,

- $H^{2} \times \mathbb{R}$ if $k_{0}=1$,

- Sol if $k_{0}=2$,

- $\mathbb{R}^{3}$ or Nil if $k_{0}=3$.

Proof. Given $\hat{g}(\cdot) \in \overline{\mathcal{O}}_{\left(k_{0}\right)}$, put $\hat{g}_{s}(t)=\frac{1}{s} \hat{g}(s t)$. We claim that the forward orbit $\left\{\hat{g}_{s}(\cdot)\right\}_{s \in[1, \infty)}$ is relatively sequentially compact in $\overline{\mathcal{O}}_{\left(k_{0}\right)}$. To see this, suppose that there is a sequence $\left\{\hat{g}_{s_{i}}(\cdot)\right\}_{i=1}^{\infty}$ having a limit $\hat{g}^{\prime}(\cdot)$. We can find a subsequence of $\left\{\left(M, g_{s}(\cdot)\right)\right\}_{s \in[1, \infty)}$ that converges to $\hat{g}^{\prime}(\cdot)$. Thus $\hat{g}^{\prime}(\cdot) \in \overline{\mathcal{O}}$. However, the number of local symmetries cannot decrease in the limit. Hence $\hat{g}^{\prime}(\cdot) \in \overline{\mathcal{O}}_{(k)}$ for some $k \geq k_{0}$. We must have $k=k_{0}$, by the definition of $k_{0}$, which proves the claim.

Let $\left\{s_{i}\right\}_{i=1}^{\infty}$ be a sequence tending to infinity such that $\lim _{i \rightarrow \infty} \hat{g}_{s_{i}}(\cdot)=\hat{g}_{\infty}(\cdot)$ for some $\hat{g}_{\infty}(\cdot) \in \overline{\mathcal{O}}_{\left(k_{0}\right)}$. Let $S$ denote the underlying orbit space of $\hat{g}_{\infty}(1)$. There is a sequence $\left\{s_{j}^{\prime}\right\}_{j=1}^{\infty}$ tending to infinity so that $\lim _{j \rightarrow \infty}\left(M, g_{s_{j}^{\prime}}(\cdot)\right)=\hat{g}_{\infty}(\cdot)$. In particular, $\lim _{j \rightarrow \infty}\left(M, \frac{1}{s_{j}^{\prime}} g\left(s_{j}^{\prime}\right)\right) \stackrel{\mathrm{GH}}{=} S$.

If $k_{0}=0$ then by Proposition 4.80, $\left(M, \hat{g}_{\infty}(\cdot)\right)$ is the Ricci flow on a manifold of constant negative sectional curvature.

If $k_{0}=1$ then $S$ is a closed two-dimensional orbifold. Taking a double cover if necessary, we can assume that $S$ is orientable. From Proposition 5.2, we can assume that the underlying étale groupoid comes from an orbifold principal $S^{1}$-bundle on $S$. (The triviality of $e$ comes from its identification with $\mathrm{H}^{1}$ of the circle fiber of the orbifold bundle $M \rightarrow S$.) By Proposition $4.80, \hat{g}_{\infty}(\cdot)$ has $\left(H^{2} \times \mathbb{R}\right)$-type and $S$ has a metric of constant curvature $-\frac{1}{2 t}$. As $M$ is the total space of an orbifold circle bundle over $S$, it follows that $M$ admits an $H^{2} \times \mathbb{R}$ or $\widetilde{\mathrm{SL}_{2}(\mathbb{R})}$-structure (using [40] if we took a double cover).

If $k_{0}=2$ then $S$ is $S^{1}$ or an interval [0,L]. Suppose first that $S=S^{1}$. Then $M$ is the total space of a $T^{2}$-fiber bundle over $S$. Let $H \in \mathrm{SL}(2, \mathbb{Z})$ be the holonomy of the fiber bundle, defined up to conjugacy. As in the proof of Lemma 6.1, the étale 
groupoid of $\hat{g}_{\infty}(\cdot)$ arises from a (twisted) principal $T^{2}$-bundle on $S^{1}$. The flat bundle $e$ over $S^{1}$ has holonomy $H \in \mathrm{SL}(2, \mathbb{Z})$. By Proposition $4.80, \hat{g}_{\infty}(\cdot)$ has Sol-type and $H$ is a hyperbolic element of $\operatorname{SL}(2, \mathbb{Z})$. Thus $M$ admits a Sol-structure.

Suppose now that $S=[0, L]$. As in the proof of Lemma $6.1, M$ is the total space of an orbifold $T^{2}$-bundle over the orbifold $[0, L]$. A double cover $\hat{M}$ of $M$ fibers over $S^{1}$. Running the previous argument on $\hat{M}$ with the pullback metric, we conclude that $\widehat{M}$ admits a Sol-structure. Hence $M$ admits a Sol-structure [40].

If $k_{0}=3$ then $S$ is a point. Hence $\left\{\left(M, \frac{1}{s_{j}^{\prime}} g\left(s_{j}^{\prime}\right)\right)\right\}_{j=1}^{\infty}$ Gromov-Hausdorff converges, with bounded sectional curvature, to a point. As in the proof of Lemma 6.1, $\hat{g}_{\infty}(\cdot)$ is locally homogeneous and has $\mathbb{R}^{3}$ or Nil as its local symmetry group. Such a Ricci flow solution is automatically a locally homogeneous expanding soliton.

We have shown that there is some sequence $\left\{s_{j}\right\}_{j=1}^{\infty}$ tending to infinity so that $\lim _{j \rightarrow \infty}\left(M, g_{s_{j}}(\cdot)\right)$ exists and is a locally homogeneous expanding soliton. We now wish to show that this is true for any sequence $\left\{s_{j}\right\}_{j=1}^{\infty}$ tending to infinity, at least if the Thurston type of $M$ is not $\widetilde{\mathrm{SL}_{2}(\mathbb{R})}$. The first step is to show that under a compactness assumption, there is a parameter $T$ so that if we take any rescaling limit $\bar{g}(\cdot)$ then upon further rescaling of $\bar{g}(\cdot)$, the result is near a locally homogeneous expanding soliton for some rescaling parameter $s \in[1, T]$.

Proposition 6.3. Given $k$, let $C$ be a sequentially compact subset of $\overline{\mathcal{O}}_{(k)}$. Let $U$ be a neighborhood of

- the $H^{3}$-type locally homogeneous expanding solitons in $\overline{\mathcal{O}}_{(0)}$ if $k=0$,

- the $\left(H^{2} \times \mathbb{R}\right)$-type locally homogeneous expanding solitons in $\overline{\mathcal{O}}_{(1)}$ if $k=1$,

- the Sol-type locally homogeneous expanding solitons in $\overline{\mathcal{O}}_{(2)}$ if $k=2$,

- the $\mathbb{R}^{3}$-type and Nil-type locally homogeneous expanding solitons in $\overline{\mathcal{O}}_{(3)}$ if $k=3$.

Then there is a $T=T(k, C, U) \in[1, \infty)$ so that for any $\bar{g}(\cdot) \in C$, if $\bar{g}_{s}(\cdot) \in C$ for all $s \in[1, T]$ then there is some $s \in[1, T]$ such that $\bar{g}_{s}(\cdot) \in U$.

Proof. Given $k, C$ and $U$, suppose that the proposition is not true. Then for each $j \in \mathbb{Z}^{+}$, there is some $\bar{g}^{(j)}(\cdot) \in C$ so that for each $s \in[1, j], \bar{g}_{s}^{(j)}(\cdot) \in C$ and $\bar{g}_{s}^{(j)}(\cdot) \notin U$. Take a convergent subsequence of the $\left\{\bar{g}^{(j)}(\cdot)\right\}_{j=1}^{\infty}$ with limit $\bar{g}^{(\infty)}(\cdot)$. Then for all $s \in[1, \infty)$, we have $\bar{g}_{s}^{(\infty)}(\cdot) \in C$ and $\bar{g}_{s}^{(\infty)}(\cdot) \notin U$. By sequential compactness, there is a sequence $\left\{t_{k}\right\}_{k=1}^{\infty}$ in $\mathbb{Z}^{+}$tending to infinity so that $\lim _{k \rightarrow \infty} \bar{g}_{t_{k}}^{(\infty)}(\cdot)$ exists and equals some $\bar{g}_{\infty}^{(\infty)}(\cdot) \in C$. By Proposition 4.80, $\bar{g}_{\infty}^{(\infty)}(\cdot)$ is a locally homogeneous expanding soliton as in the statement of the present proposition. Then for large $k$, we have $\bar{g}_{t_{k}}^{(\infty)}(\cdot) \in U$, which is a contradiction. 
The next step is to use local stability to say that after rescaling $\bar{g}(\cdot)$ by the parameter $T$, the result is definitely near a locally homogeneous expanding soliton solution.

Proposition 6.4. Suppose that $M$ does not have Thurston type $\widetilde{\mathrm{SL}_{2}(\mathbb{R})}$. Then there are decreasing open sets $\left\{U_{l}\right\}_{l=1}^{\infty}$ of the type described in Proposition 6.3, whose intersection is the corresponding set of locally homogeneous expanding soliton solutions, so that under the hypotheses of Proposition 6.3, if $T_{l}=T\left(k, C, U_{l}\right)$ then we are ensured that $\bar{g}_{T_{l}}(\cdot) \in U_{l}$. (In the case $k=1$ we restrict to Ricciflow solutions on an étale groupoid with vanishing Euler class, so $U_{l}$ is a neighborhood in the relative topology.)

Proof. This follows from the local stability of the expanding solitons in $\overline{\mathcal{O}}_{(k)}$. That is, there is a sequence $\left\{U_{l}\right\}_{l=1}^{\infty}$ of such neighborhoods so that $\bar{g}_{s}(\cdot) \in U_{l}$ implies that $\bar{g}_{s^{\prime}}(\cdot) \in U_{l}$ whenever $s^{\prime} \geq s$. (In fact, one has exponential convergence to the set of expanding solitons.) The case $k=0$ appears in [54]. The case $k=2$ appears in [34]. In the case $k=1$, recall from Example 5.10 that there are two relevant types of étale groupoids, one with vanishing Euler class and one with nonvanishing Euler class. The locally homogeneous expanding solitons live on étale groupoids with vanishing Euler class. Their local stability (modulo the center manifold), among Ricci flows on étale groupoids with vanishing Euler class, is shown in [34]. We remark that if $M$ has Thurston type $H^{2} \times \mathbb{R}$ then a $\operatorname{limit}_{\lim _{j \rightarrow \infty}}\left(M, g_{s_{j}}(\cdot)\right)$ can only be a Ricci flow on an étale groupoid with vanishing Euler class.

Note that if $k=1$ then there may be a moduli space of locally homogeneous expanding solitons of type $H^{2} \times \mathbb{R}$ in $\overline{\mathcal{O}}_{(1)}$, corresponding to various metrics of constant curvature $-\frac{1}{2}$ on the orbit space. However, because of our diameter bound, the moduli space is compact. Comparing with [34], it may appear that there is also a factor in the moduli space consisting of harmonic 1-forms on the orbit space. However, by Lemma 5.11, the various harmonic 1 -forms all give equivalent geometries.

Remark 6.5. There is no locally homogeneous expanding soliton solution on a threedimensional étale groupoid of the type considered in Proposition 6.2 if it has an orbifold surface base with negative Euler characteristic, and a nonvanishing Euler class. A Ricci flow on such an étale groupoid will have a rescaling sequence that converges to an $\left(H^{2} \times \mathbb{R}\right)$-type expander on an étale groupoid with vanishing Euler class.

In order to show convergence of the Ricci flow on a 3-manifold with Thurston type $\widetilde{\mathrm{SL}_{2}(\mathbb{R})}$, at least by our methods, one would have to show that the expanding solitons of type $H^{2} \times \mathbb{R}$ are also locally stable if one considers neighborhoods that include étale groupoids with nonvanishing Euler class. The difficulty is that the nearby Ricci flows live on an inequivalent groupoid and so one cannot just linearize around the $\left(H^{2} \times \mathbb{R}\right)$-type expanding solitons. One approach would be to instead consider Ricci 
flows with $\widetilde{\mathrm{SL}_{2}(\mathbb{R})}$-symmetry on étale groupoids with nonzero Euler class and show that this finite-dimensional family is an attractor.

We now show if $k_{0}<3$ and $M$ does not have Thurston type $\widetilde{\mathrm{SL}_{2}(\mathbb{R})}$ then any rescaling limit $\bar{g}(\cdot)$ is a locally homogenous expanding soliton. The method of proof is to show that we can rescale $\bar{g}(\cdot)$ backward by a factor $T$, and then apply the previous proposition.

Proposition 6.6. If $k_{0}<3$ and $M$ does not have Thurston type $\widetilde{\mathrm{SL}_{2}(\mathbb{R})}$ then for any sequence $\left\{s_{j}\right\}_{j=1}^{\infty}$ tending to infinity, as $j \rightarrow \infty,\left(M, g_{s_{j}}(\cdot)\right)$ approaches the set of locally homogeneous expanding solitons of the type listed in Proposition 6.3, with $k=k_{0}$.

Proof. If the proposition is not true then there is a sequence $\left\{s_{j}\right\}_{j=1}^{\infty}$ tending to infinity and a neighborhood $U_{l}$ as in Proposition 6.4 so that for all $j, g_{s_{j}}(\cdot) \notin U_{l}$. After passing to a further subsequence, we can assume that $\lim _{j \rightarrow \infty} g_{s_{j}}(\cdot)=\bar{g}(\cdot)$ for some $\bar{g}(\cdot) \in \overline{\mathcal{O}}$.

If $k_{0}=2$ then from Proposition 6.2, $M$ admits a Sol-structure. As $M$ cannot collapse with bounded curvature and bounded diameter to something of dimension other than one, $\overline{\mathcal{O}}_{(0)}=\overline{\mathcal{O}}_{(1)}=\overline{\mathcal{O}}_{(3)}=\emptyset$. Then $C=\overline{\mathcal{O}}_{(2)}$ is sequentially compact and $\bar{g}(\cdot) \in \overline{\mathcal{O}}_{(2)}$. A similar argument applies in the other cases when $k_{0}<3$ to show that $C=\overline{\mathcal{O}}_{\left(k_{0}\right)}$ is sequentially compact and $\bar{g}(\cdot) \in \overline{\mathcal{O}}_{\left(k_{0}\right)}$.

For $s \geq 1$, let $\bar{g}^{\left(s^{-1}\right)}(\cdot)$ be the limit in $\overline{\mathcal{O}}$ of a convergent subsequence of $\left\{g_{s^{-1} s_{j}}(\cdot)\right\}_{j=1}^{\infty}$. Then $\bar{g}(\cdot)=\bar{g}_{s}^{\left(s^{-1}\right)}(\cdot)$. Note that $\bar{g}^{\left(s^{-1}\right)}(\cdot) \in \overline{\mathcal{O}}_{k_{0}}$. By Proposition 6.4 , there is a number $T_{l} \geq 1$ so that for each $s \geq 1, \bar{g}_{T_{l}}^{\left(s^{-1}\right)}(\cdot) \in U_{l}$. Taking $s=T_{l}$, we conclude that $\bar{g}(\cdot) \in U_{l}$. This is a contradiction.

Corollary 6.7. If $k_{0}=0$ or $k_{0}=2$ then $\lim _{s \rightarrow \infty}\left(M, g_{s}(\cdot)\right)$ exists and is one of the locally homogeneous expanding solitons of the type listed in Proposition 6.3, with $k=k_{0}$.

Proof. In these cases, given $M$, there is a unique locally homogeneous expanding soliton of the type listed in Proposition 6.3, with $k=k_{0}$. The relationship between the topology of $M$ and the equivalence class of the étale groupoid comes from the proof of Lemma 6.1. If $k_{0}=0$ then $M$ admits a hyperbolic metric and the expander is the solution $\bar{g}(t)=4 t g_{\text {hyp }}$, where $g_{\text {hyp }}$ is the metric of constant sectional curvature -1 on $M$. If $k_{0}=2$ then $M$ is a Sol-manifold. Suppose first that $M$ is the total space of a $T^{2}$-bundle over $S^{1}$, with hyperbolic holonomy $H \in \operatorname{SL}(2, \mathbb{Z})$. Then by Remark 4.82, the expander can be written $\bar{g}=\frac{t}{2} \operatorname{Tr}\left(X^{2}\right) d b^{2}+(d y)^{T} e^{b X} d y$, where $b \in[0,1]$ and $e^{X}=H^{T} H$. If $M$ fibers over the orbifold $[0,1]$ then the expander is a $\mathbb{Z}_{2}$-quotient thereof. 
In the case $k_{0}=3$, we must show that any rescaling limit $\bar{g}(\cdot)$ has three local symmetries. This does not follow just from topological arguments. The method of proof is to rescale backwards and then apply the monotonicity arguments of Section 4 to a backward limit.

Proposition 6.8. If $k_{0}=3$ and $\bar{g}(\cdot) \in \overline{\mathcal{O}}$ is a limit $\lim _{j \rightarrow \infty}\left(M, g_{s_{j}}(\cdot)\right)$, for some sequence $\left\{s_{j}\right\}_{j=1}^{\infty}$ tending to infinity, then $\bar{g}(\cdot) \in \overline{\mathcal{O}}_{(3)}$.

Proof. Suppose that $\bar{g}(\cdot) \in \overline{\mathcal{O}}_{(k)}$ with $k<3$. As in the proof of Proposition 6.6, for $s \geq 1$, let $\bar{g}^{\left(s^{-1}\right)}(\cdot)$ be the limit in $\overline{\mathcal{O}}$ of a convergent subsequence of $\left\{g_{s^{-1}} s_{j}(\cdot)\right\}_{j=1}^{\infty}$. Then $\bar{g}(\cdot)=\bar{g}_{s}^{\left(s^{-1}\right)}(\cdot)$. More precisely, for each $s \in[1, \infty)$ there is an equivalence $\phi_{s}$ of groupoids so that

$$
\bar{g}(t)=\frac{1}{s} \phi_{s}^{*} \bar{g}^{\left(s^{-1}\right)}(s t) .
$$

In particular, $\bar{g}^{\left(s^{-1}\right)}(\cdot) \in \overline{\mathcal{O}}_{(k)}$. Using (6.9), we can extend the domain of definition of $\bar{g}(\cdot)$ to $\left[s^{-1}, \infty\right)$ for all $s \geq 1$, and hence to all $t \in(0, \infty)$. We still have the bounds $\sup _{t \in(0, \infty)} t\|\operatorname{Riem}(\bar{g}(t))\|_{\infty} \leq K$ and $\sup _{t \in(0, \infty)} t^{-\frac{1}{2}} \operatorname{diam}(\bar{g}(t)) \leq D$.

As in the proof of Proposition 4.79, we construct a solution $f(t)$ of the conjugate heat equation on the orbit space $S$,

$$
\begin{aligned}
\frac{\partial\left(e^{-f}\right)}{\partial t}=-\nabla^{2} e^{-f}+(R- & \frac{1}{4} g^{\alpha \beta} G^{i j} G_{j k, \alpha} G^{k l} G_{l i, \beta} \\
& \left.-\frac{1}{2} g^{\alpha \gamma} g^{\beta \delta} G_{i j} F_{\alpha \beta}^{i} F_{\gamma \delta}^{j}+\frac{n}{2 t}\right) e^{-f},
\end{aligned}
$$

where $n=\operatorname{dim}(S)=3-k$, that satisfies $(4 \pi t)^{-\frac{n}{2}} \int_{S} e^{-f} \operatorname{dvol}_{S}=1$ for all $t \in(0, \infty)$. Then $\mathcal{W}_{+}\left(G_{i j}(t), A_{\alpha}^{i}(t), g_{\alpha \beta}(t), f(t), t\right)$ is nondecreasing in $t$. From Lemma 6.1, for $\mathrm{t}<1$ there is a uniform positive lower bound on $t^{-\frac{n}{2}} \operatorname{vol}\left(S, g_{\alpha \beta}(t)\right)$. By O'Neill's theorem, the lower sectional curvature bound on $\bar{g}(t)$ implies the same lower sectional curvature bound on $g_{\alpha \beta}(t)$. Hence the (orbifolds) $\left(S, t^{-1} g_{\alpha \beta}(t)\right)$ are noncollapsing in the Gromov-Hausdorff sense as $t \rightarrow 0$. It follows that $\left\{\bar{g}^{s^{-1}}(\cdot)\right\}_{s \geq 1}$ lies in a sequentially compact subset of $\overline{\mathcal{O}}_{(k)}$, since if a sequence $\left\{\bar{g}^{s_{r}-1}(\cdot)\right\}_{r=1}^{\infty}$ with $\lim _{r \rightarrow \infty} s_{r}=\infty$ converged to an element of $\overline{\mathcal{O}}_{\left(k^{\prime}\right)}$ with $k^{\prime}>k$ then the orbit spaces $\left\{\left(S, s_{r} g_{\alpha \beta}\left(s_{r}^{-1}\right)\right)\right\}_{j=1}^{\infty}$ would Gromov-Hausdorff converge to something of dimension $3-k^{\prime}<3-k$, which contradicts the noncollapsing. In particular, $t^{-\frac{n}{2}} \operatorname{vol}\left(S, g_{\alpha \beta}(t)\right)$ is uniformly bounded above as $t \rightarrow 0$ (as also follows from the diameter and lower curvature bounds). Then from Lemma 4.61, $W_{+}\left(G_{i j}(t), A_{\alpha}^{i}(t), g_{\alpha \beta}(t), f(t), t\right)$ is uniformly bounded from below as $t \rightarrow 0$. There is a sequence of times $t_{j} \rightarrow 0$ so that

$$
\left.\lim _{j \rightarrow \infty} t_{j} \frac{d}{d t}\right|_{t=t_{j}} W_{+}\left(G_{i j}(t), A_{\alpha}^{i}(t), g_{\alpha \beta}(t), f(t), t\right)=0 .
$$


After passing to a subsequence, we can assume that $\lim _{j \rightarrow \infty} \bar{g}^{t}(\cdot)=\bar{g}_{0}(\cdot)$ for some $\bar{g}_{0}(\cdot) \in \overline{\mathcal{O}}_{(k)}$, defined for $t \in(0, \infty)$. As in the proof of Proposition 4.79, for any $t \in(0, \infty)$ the measures $\left(4 \pi t_{j} t\right)^{-\frac{n}{2}} e^{-f\left(t_{j} t\right)} \mathrm{dvol}\left(S, g_{\alpha \beta}\left(t_{j} t\right)\right)$ will subconverge to a smooth positive probability measure on $S$. Using (4.66), we get that $\bar{g}_{0}(\cdot)$ satisfies the conclusion of Proposition 4.67 at time $t=1$. It follows that $\bar{g}_{0}(\cdot)$ satisfies the conclusion of Proposition 4.67 for all $t \geq 1$. In particular, $M$ admits a geometric structure other than an $\mathbb{R}^{3}$ or a Nil-structure (see the proof of Proposition 6.2), which is a contradiction.

Proposition 6.11. If $k_{0}=3$ then for any sequence $\left\{s_{j}\right\}_{j=1}^{\infty}$ tending to infinity, $\lim _{j \rightarrow \infty} g_{s_{j}}(\cdot)$ exists and is a locally homogeneous expanding soliton of the $\mathbb{R}^{3}$ or Nil-type.

Proof. If the proposition is not true then there is a sequence $\left\{s_{j}\right\}_{j=1}^{\infty}$ tending to infinity such that $\lim _{j \rightarrow \infty} g_{s_{j}}(\cdot)=\bar{g}(\cdot)$ for some $\bar{g}(\cdot) \in \overline{\mathcal{O}}$, but $\bar{g}(\cdot)$ is not an expander of type $\mathbb{R}^{3}$ or Nil. From Proposition $6.8, \bar{g}(\cdot) \in \overline{\mathcal{O}}_{(3)}$. In particular, $\bar{g}(\cdot)$ is locally homogeneous. If $\underline{g}$ is a local system of $\mathbb{R}^{3}$ Lie algebras then $\bar{g}(\cdot)$ must be flat. If $\underline{g}$ is a local system of $\bar{n}$ il Lie algebras then $\bar{g}(\cdot)$ is automatically a locally homogeneous expanding soliton, with respect to some origin of time. A priori, the equation for $\bar{g}(\cdot)$ could differ from the expanding Nil soliton in Theorem 1.2 by an additive change of the time parameter. We can rule this out by using stability arguments as before, which are simpler in this case because we are now talking about dynamics on the finitedimensional space of locally homogenous Nil-solutions. First, we argue that there is some sequence $s_{j} \rightarrow \infty$ so that $\lim _{j \rightarrow \infty} g_{s_{j}}(\cdot)$ is a locally homogeneous expanding soliton modeled on the Nil expanding soliton of Theorem 1.2. Then we use the fact that this expanding soliton is an attractor for the $\mathbb{R}^{\geq 1}$-semigroup action on the locally homogeneous Nil-solutions [38, Section 3.3.3]. Finally, we use a backward rescaling, as in the proof of Proposition 6.8, to show that for any sequence $\left\{s_{j}\right\}_{j=1}^{\infty}$ tending to infinity, $\lim _{j \rightarrow \infty} g_{s_{j}}(\cdot)$ is a locally homogeneous expanding soliton modeled on the Nil expanding soliton of Theorem 1.2

Remark 6.12. Some of the results of this subsection extend to higher dimension. Suppose that $(M, g(\cdot))$ is a Ricci flow on a closed $n$-dimensional manifold that exists for $t \in(1, \infty)$, with sectional curvatures that are uniformly $O\left(t^{-1}\right)$ and diameter that grows at most like $O\left(t^{\frac{1}{2}}\right)$. (If $n>3$ then not all compact Ricci flows satisfy these assumptions, as seen by the static solution on a Ricci-flat $K 3$ surface.) If $\left\{s_{j}\right\}_{j=1}^{\infty}$ is any sequence tending to infinity then after passing to a subsequence, there is a limit Ricci flow $\bar{g}(\cdot)$ on an $n$-dimensional étale groupoid $\$$. If $M$ is aspherical then $\$$ is locally free.

If $n$ is greater than three then the first point is that the local symmetry sheaf g may be a sheaf of nonabelian nilpotent Lie algebras. (This could also happen 
in dimension 3, but then $\bar{g}(\cdot)$ is locally homogeneous with respect to the threedimensional Heisenberg group.) Thus the analysis of Section 4.2 would have to be extended to the case of twisted $\mathcal{E}$-bundles where $\mathcal{E}$ is a nilpotent Lie group.

If we do assume that $\mathrm{g}$ is abelian then Proposition 4.79 says that any blowdown limit of $\bar{g}(\cdot)$ satisfies the harmonic-Einstein equations (4.68). Proposition 4.80 describes the blowdown limit of a Ricci flow solution $(M, g(\cdot))$ on an aspherical 4manifold, defined for $t \in(1, \infty)$, with sectional curvatures that are uniformly $O\left(t^{-1}\right)$ and diameter which is $O\left(t^{\frac{1}{2}}\right)$, provided that $\underline{\mathrm{g}}$ is abelian.

6.2. Proof of Theorem 1.2. In this subsection we use the fact that $M$ is aspherical in order to extend the convergence result of Section 6.1 from a statement about a limiting Ricci flow on an étale groupoid to a statement about a limiting Ricci flow on $\tilde{M}$.

By Proposition 3.5, $M$ is irreducible, aspherical and has a single geometric piece in its geometric decomposition. We assume first that $M$ does not have Thurston type $\widetilde{\mathrm{SL}_{2}(\mathbb{R})}$.

Suppose that there is a sequence $\left\{s_{j}\right\}_{j=1}^{\infty}$ tending to infinity such that the limit $\lim _{j \rightarrow \infty}\left(M, g_{s_{j}}(\cdot)\right)$ exists and equals a Ricci flow $\bar{g}(\cdot)$ on an étale groupoid $G$. If $S$ is the orbit space of $(G, \bar{g}(1))$ then $\lim _{j \rightarrow \infty}\left(M, \frac{g\left(s_{j}\right)}{s_{j}}\right) \stackrel{\mathrm{GH}}{=} S$.

From Propositions 6.6 and $6.11, \bar{g}(\cdot)$ is a locally homogeneous expanding soliton of the type listed in Proposition 6.3. There is an orbifold fiber bundle $M \rightarrow S$. Now $S$ is a very good orbifold, i.e., $S$ is the quotient of a manifold $\widehat{S}$ by a finite group action. Taking the corresponding finite cover $\hat{M}$ of $M$, if we are interested in what happens on the universal cover $\widetilde{M}$ then we can assume that $S$ is a closed manifold.

Suppose that $M$ is not of Nil-type. For large $j$, we know that $\left(M, \frac{g\left(s_{j}\right)}{s_{j}}\right)$ is the total space of a $T^{k_{0}}$-bundle over $S$ which defines an $F$-structure, where $k_{0}=$ $\operatorname{dim}(M)-\operatorname{dim}(S)$. As $M$ is aspherical, the map $\pi_{1}\left(T^{k_{0}}\right) \rightarrow \pi_{1}(M)$ is injective [7, Remark 0.9].

Choose $\delta \in\left(0, \min \left(\frac{\operatorname{inj}(S)}{10}, \frac{1}{10 \sqrt{K}}\right)\right)$ and take a finite collection $\left\{x_{i}\right\}$ of points in $S$ with the property that $\left\{B\left(x_{i}, \delta\right)\right\}$ covers $S$. For large $j$, let $\left\{p_{i, j}\right\}$ be points in $\left(M, \frac{g\left(s_{j}\right)}{s_{j}}\right)$ that are the image of $\left\{x_{i}\right\}$ under a Gromov-Hausdorff approximation. Then for such $j,\left\{B\left(p_{i, j}, 5 \delta\right)\right\}$ covers $\left(M, \frac{g\left(s_{j}\right)}{s_{j}}\right)$. Each $B\left(p_{i, j}, \delta\right)$ is homeomorphic to $B^{3-k_{0}} \times T^{k_{0}}$ and its lift $\widetilde{B\left(p_{i, j}, \delta\right)}$ to $\tilde{M}$ is homeomorphic to $B^{3-k_{0}} \times \mathbb{R}^{k_{0}}$.

Suppose that $\tilde{p}_{i, j} \in \tilde{M}$ is a preimage of $p_{i, j}$. Then the $5 \delta$-ball $B(0,5 \delta) \subset T_{p_{i, j}} M$ is isometric to $B\left(\tilde{p}_{i, j}, 5 \delta\right) \subset\left(\tilde{M}, \frac{\tilde{g}\left(s_{j}\right)}{s_{j}}\right)$ with respect to the metric $\exp _{p_{i, j}}^{*} \frac{g\left(s_{j}\right)}{s_{j}}$. From the construction of the Riemannian groupoid ( $\mathbb{G}, \bar{g}(1))$ [38, Proposition 5.9], $\lim _{j \rightarrow \infty} B\left(\tilde{p}_{i, j}, 5 \delta\right)$ is isometric to a $5 \delta$-ball in the time-1 slice of the (homogeneous) expanding soliton solution on the manifold $\mathbb{R}^{3}$. 
Let $\tilde{m} \in \tilde{M}$ be a basepoint. Given $R>0$, consider $B(\tilde{m}, R) \subset\left(\tilde{M}, \frac{\tilde{g}\left(s_{j}\right)}{s_{j}}\right)$. For large $j$, we can find a finite collection of points $\left\{\widetilde{p}_{r}\right\}$ (depending on $j$ ) in $\left(\tilde{M}, \frac{\tilde{g}\left(s_{j}\right)}{s_{j}}\right)$, where each $\tilde{p}_{r}$ projects to some element of $\left\{p_{i, j}\right\} \subset M$, so that the cardinality of $\left\{\widetilde{p}_{r}\right\}$ is uniformly bounded in $j$ and $B(\tilde{m}, R) \subset\left(\tilde{M}, \frac{\tilde{g}\left(s_{j}\right)}{s_{j}}\right)$ is covered by $\left\{B\left(\widetilde{p}_{r}, 5 \delta\right)\right\}$. Namely, for each $i$ and $j$, take points in the strip $\widehat{B\left(p_{i, j}, \delta\right)} \subset\left(\tilde{M}, \frac{\tilde{g}\left(s_{j}\right)}{s_{j}}\right)$ that lie in $B(\tilde{m}, R)$, cover $p_{i, j}$ and form a separated net of size approximately $\delta$.

After relabeling the indices if necessary, suppose that $\tilde{m} \in B\left(\widetilde{p}_{1}, 5 \delta\right)$ with $\widetilde{p}_{1} \in \tilde{M}$ projectioning to $p_{1, j} \in M$. For large $j$, fix an almost-isometry from $B\left(\tilde{p}_{1}, 5 \delta\right)$ to a $5 \delta$-ball in the time-1 slice of the (homogeneous) expanding soliton solution on $\mathbb{R}^{3}$. Taking the union of the balls $B\left(\tilde{p}_{r}, 5 \delta\right)$ whose centers project to $p_{1, j} \in M$, it follows that for large $j$, the metric $\frac{\tilde{g}\left(s_{j}\right)}{s_{j}}$ on $\overline{B\left(p_{1, j}, \delta\right)} \cap B(\tilde{m}, R)$ approaches the homogeneous expanding soliton metric on the strip. We do the same procedure for the other values of $i$, on the strips $\left.\widehat{B\left(p_{i, j}, \delta\right.}\right) \cap B(\tilde{m}, R) \subset\left(\tilde{M}, \frac{\tilde{g}\left(s_{j}\right)}{s_{j}}\right)$. Then taking the union of these strips for the various $i$, it follows that for large $j$, the metric $\frac{\tilde{g}\left(s_{j}\right)}{s_{j}}$ on $B(\tilde{m}, R)$ approaches an $R$-ball in the time-one slice of the homogeneous expanding soliton solution on $\mathbb{R}^{3}$. Finally, we can perform the argument with the time parameter added, to conclude $\left\{\left(\tilde{M}, \tilde{m}, \tilde{g}_{s_{j}}(\cdot)\right)\right\}_{j=1}^{\infty}$ converges to the expanding soliton solution on $\mathbb{R}^{3}$, in the topology of pointed smooth convergence. We can perform a similar argument in the Nil-case, where $S$ is a point.

To prove Theorem 1.2, suppose first that $M$ has Thurston type $\mathbb{R}^{3}$, Nil, Sol or $H^{3}$. Suppose that the theorem is not true. Then there is a sequence $\left\{s_{j}\right\}_{j=1}^{\infty}$ tending to infinity so that for any subsequence $\left\{s_{j_{r}}\right\}_{r=1}^{\infty}$, either $\left\{\left(M, g_{s_{j r}}(1)\right)\right\}_{r=1}^{\infty}$ does not converge in the Gromov-Hausdorff topology to the limit stated in the theorem or $\left\{\left(\tilde{M}, \tilde{m}, \tilde{g}_{s_{j_{r}}}(\cdot)\right)\right\}_{r=1}^{\infty}$ does not converge in the pointed smooth topology to the homogeneous expanding soliton solution stated in the theorem. After passing to a subsequence, there is a $\operatorname{limit}_{\lim _{j \rightarrow \infty}}\left(M, g_{s_{j}}(\cdot)\right)$ as a Ricci flow on an étale groupoid, whose time-one orbit space will be the Gromov-Hausdorff $\operatorname{limit}_{\lim } \rightarrow \infty\left(M, \frac{g\left(s_{j}\right)}{s_{j}}\right)$. The limit is characterized by Corollary 6.7 and Proposition 6.11. From the preceding discussion, there is a pointed smooth $\operatorname{limit}_{j \rightarrow \infty}\left(\tilde{M}, \tilde{m}, \tilde{g}_{s_{j}}(\cdot)\right)$ as a Ricci flow on $\tilde{M}$, which is a homogeneous expanding soliton solution on $\mathbb{R}^{3}$ of the corresponding type. In any case, we get a contradiction.

Suppose now that $M$ has Thurston type $H^{2} \times \mathbb{R}$. We can apply the same argument. The only difference is that we can no longer say that $\lim _{s \rightarrow \infty}\left(M, g_{s}(1)\right)$ exists in the Gromov-Hausdorff topology. All that we get from Proposition 6.6 is that for any sequence $\left\{t_{j}\right\}_{j=1}^{\infty}$ tending to infinity, there is a subsequence $\left\{t_{j_{r}}\right\}_{r=1}^{\infty}$ for which $\lim _{r \rightarrow \infty}\left(M, \frac{g\left(t_{j_{r}}\right)}{t_{j_{r}}}\right)$ exists and equals a closed 2-dimensional orbifold with constant 
sectional curvature $-\frac{1}{2}$. A priori, different subsequences could give rise to to different constant-curvature orbifolds. (From our diameter bound, for a given $M$ there is a compact set of such orbifolds that can arise). However, we claim that on the universal cover $\tilde{M}$ we do get pointed smooth convergence $\lim _{s \rightarrow \infty}\left(\tilde{M}, \tilde{m}, \tilde{g}_{s}(\cdot)\right)$ to the Ricci flow $\left(H^{2} \times \mathbb{R}, 2 t g_{\text {hyp }}+g_{\mathbb{R}}\right)$. To see this, suppose that $\left\{s_{j}\right\}_{j=1}^{\infty}$ is a sequence tending to infinity so that for any subsequence $\left\{s_{j_{r}}\right\}_{r=1}^{\infty},\left\{\left(\tilde{M}, \tilde{g}_{s_{r}}(\cdot)\right)\right\}_{r=1}^{\infty}$ does not converge to $\left(H^{2} \times \mathbb{R}, 2 t g_{\text {hyp }}+g_{\mathbb{R}}\right)$ in the pointed smooth topology. We know that there is a subsequence $\left\{s_{j_{r}}\right\}_{r=1}^{\infty}$ for which $\lim _{r \rightarrow \infty}\left(M, g_{s_{j_{r}}}(\cdot)\right)$ exists and is a Ricci flow $\bar{g}(\cdot)$ on an étale groupoid. From Proposition 6.6, $\bar{g}(\cdot)$ is a Ricci flow solution of type $H^{2} \times \mathbb{R}$. From the preceding discussion, $\lim _{r \rightarrow \infty}\left(\tilde{M}, \tilde{m}, \tilde{g}_{s_{j_{r}}}(\cdot)\right)$ exists in the pointed smooth topology and equals the expanding soliton solution $\left(H^{2} \times \mathbb{R}, 2 t g_{\text {hyp }}+g_{\mathbb{R}}\right)$. This is a contradiction.

Finally, if $M$ has Thurston type $\widetilde{\mathrm{SL}_{2}(\mathbb{R})}$ then the theorem follows from Proposition 6.2.

Remark 6.13. The assumption $\operatorname{diam}(M, g(t))=O\left(t^{\frac{1}{2}}\right)$ of Theorem 1.2, together with the curvature assumption, ensures that $M$ has a single geometric piece. One can ask what happens if one removes the diameter assumption but keeps the curvature assumption. In such a case one would clearly have to consider pointed limits $\lim _{j \rightarrow \infty}\left(M, m, g_{s_{j}}(\cdot)\right)$ of the Ricci flow solution. After passing to a subsequence, there will be convergence to a Ricci flow solution $\bar{g}(\cdot)$ on a pointed étale groupoid. However, the analysis of Section 4.2 does not immediately extend to the pointed noncompact setting. For example, $\bar{g}(t)$ need not have finite volume in any reasonable sense; see Example 2.3.

\section{References}

[1] L. Bessières, G. Besson, M. Boileau, S. Maillot, and J. Porti, Collapsing irreducible 3-manifolds with nontrivial fundamental group. Invent. Math. 179 (2010), 435-460. Zbl 05661760 MR 2570121

[2] M. Bridson and A. Haefliger, Metric spaces of non-positive curvature. Grundlehren Math. Wiss. 319, Springer-Verlag, Berlin 1999. Zbl 0988.53001 MR 1744486

[3] J. Cheeger, K. Fukaya, and M. Gromov, Nilpotent structures and invariant metrics on collapsed manifolds. J. Amer. Math. Soc. 5 (1992), 327-372. Zbl 0758.53022 MR 1126118

[4] J. Cheeger and D. Gromoll, The splitting theorem for manifolds of nonnegative Ricci curvature. J. Differential Geom. 6 (1971), 119-128. Zbl 0223.53033 MR 0303460

[5] J. Cheeger and M. Gromov, Collapsing Riemannian manifolds while keeping their curvature bounded I. J. Differential Geom. 23 (1986), 309-346. Zbl 0606.53028 MR 0852159

[6] J. Cheeger and M. Gromov, Collapsing Riemannian manifolds while keeping their curvature bounded II. J. Differential Geom. 32 (1990), 269-298. Zbl 0727.53043 MR 1064875 
[7] J. Cheeger and X. Rong, Collapsed Riemannian manifolds with bounded diameter and bounded covering geometry. Geom. Funct. Anal. 5 (1995), 141-163. Zbl 0843.53039 MR 1334865

[8] B. Chow, S.-C. Chu, D. Glickenstein, C. Guenther, J. Isenberg, T. Ivey, D. Knopf, P. Lu, F. Luo, and L. Ni, The Ricci Flow: Techniques and Applications, Part I : Geometric Aspects. Math. Surveys Monogr. 135, Amer. Math. Soc., Providence, R.I., 2007. Zbl 05149301 MR 2302600

[9] B. Chow, D. Glickenstein, and P. Lu, Collapsing sequences of solutions to the Ricci flow on 3-manifolds with almost nonnegative curvature. Math. Z. 254 (2006), 1-28. Zbl 1103.53038 MR 2232005

[10] T. Colding and W. Minicozzi, Estimates for the extinction time for the Ricci flow on certain 3-manifolds and a question of Perelman. J. Amer. Math. Soc. 18 (2005), 561-569. Zbl 1083.53058 MR 2138137

[11] T. Colding and W. Minicozzi, Width and finite extinction time of Ricci flow. Geom. Topol. 12 (2008), 2537-2586. Zbl 1161.53352 MR 2460871

[12] K. Corlette, Flat G-bundles with canonical metrics. J. Differential Geom. 28 (1988), 361-382. Zbl 0676.58007 MR 0965220

[13] D. DeTurck, Deforming metrics in the direction of their Ricci tensors. J. Differential Geom. 18 (1983), 157-162. Zbl 0517.53044 MR 0697987

[14] M. Feldman, T. Ilmanen, and L. Ni, Entropy and reduced distance for Ricci expanders. $J$. Geom. Anal. 15 (2005), 49-62. Zbl 1071.53040 MR 2132265

[15] J. Frenkel, Cohomologie non abélienne et espaces fibrés. Bull. Soc. Math. France 85 (1957), 135-220. Zbl 0082.37702 MR 0098200

[16] K. Fukaya, Hausdorff convergence of Riemannian manifolds and its applications. In Recent topics in differential and analytic geometry, Adv. Stud. Pure Math. 18, Academic Press, Boston 1990, 143-238. Zbl 0754.53004 MR 1145256

[17] K. Fukaya, Margulis' lemma in Riemannian geometry. Sugaku Expositions 6 (1993), 201-219. Zbl 0796.53048 MR 1254036

[18] D. Glickenstein, Precompactness of solutions to the Ricci flow in the absence of injectivity radius estimates. Geom. Topol. 7 (2003), 487-510. Zbl 1044.53048 MR 2026540

[19] M. Gromov, Almost flat manifolds. J. Differential Geom. 13 (1978), 231-241. Zbl 0432.53020 MR 0540942

[20] M. Gromov, Metric structures for Riemannian and non-Riemannian spaces, Progr. Math. 152, Birkhäuser, Boston 1999. Zbl 0953.53002 MR 1699320

[21] C. Guenther, J. Isenberg, and D. Knopf, Linear stability of homogeneous Ricci solitons. Internat. Math. Res. Notices 2006 (2006), Art. ID 96253. Zbl 1126.53040 MR 2264732

[22] A. Haefliger, Pseudogroups of local isometries. In Differential geometry, Res. Notes in Math. 131, Pitnam, Boston, Mass., 1985. 174-197. Zbl 0656.58042 MR 0864868

[23] A. Haefliger and E. Salem, Pseudogroupes d'holonomie des feuilletages riemanniens sur des variétés compactes 1-connexes. In Géométrie Différentielle (Paris 1986), Travaux en cours 33, Hermann, Paris 1988, 141-160. Zbl 0647.57019 MR 0955853

[24] R. Hamilton, Three-manifolds with positive Ricci curvature. J. Differential Geom. 17 (1982), 255-306. Zbl 0504.53034 MR 0664497 
[25] R. Hamilton, The formation of singularities in the Ricci flow. In Surveys in differential geometry II, International Press, Cambridge, Mass., 1995, 7-136. Zbl 0867.53030 MR 1375255

[26] R. Hamilton, A compactness property for solutions of the Ricci flow. Amer. J. Math. 117 (1995), 545-572. Zbl 0840.53029 MR 1333936

[27] R. Hamilton, Non-singular solutions of the Ricci flow on three-manifolds. Comm. Anal. Geom. 7 (1999), 695-729. Zbl 0939.53024 MR 1714939

[28] R. Hamilton and J. Isenberg, Quasi-convergence of Ricci flow for a class of metrics. Comm. Anal. Geom. 1 (1993), 543-559. Zbl 0841.53058 MR 1266479

[29] J. Isenberg and M. Jackson, Ricci flow of locally homogeneous geometries on closed manifolds. J. Differential Geom. 35 (1992), 723-741. Zbl 0808.53044 MR 1163457

[30] J. Jost and K. Zuo, Harmonic maps of infinite energy and rigidity results for representations of fundamental groups of quasiprojective varieties. J. Differential Geom. 47 (1997), 469-503. Zbl 0911.58012 MR 1617644

[31] B. Kleiner and J. Lott, Notes on Perelman's papers. Geom. Topol. 12 (2008), 2587-2855. Zbl 05530173 MR 2460872

[32] B. Kleiner and J. Lott, Locally collapsed 3-manifolds. To appear.

[33] D. Knopf, Quasi-convergence of the Ricci flow. Comm. Anal. Geom. 8 (2000), 375-391. Zbl 1027.53075 MR 1753322

[34] D. Knopf, Convergence and stability of locally $\mathbb{R}^{N}$-invariant solutions of Ricci flow. $J$. Geom. Anal. 19 (2009), 817-846. MR 2538938

[35] D. Knopf and K. McLeod, Quasi-convergence of model geometries under the Ricci flow. Comm. Anal. Geom. 9 (2001), 879-919. Zbl 1020.53044 MR 1868923

[36] F. Labourie, Existence d'applications harmoniques tordues à valeurs dans les variétés à courbure négative. Proc. Amer. Math. Soc. 111 (1991), 877-882. Zbl 0783.58016 MR 1049845

[37] B. List, Evolution of an extended Ricci flow system. Comm. Anal. Geom. 16 (2008), 1007-1048. Zbl 1166.53044 MR 2471366

[38] J. Lott, On the long-time behavior of type-III Ricci flow solutions. Math. Ann. 339 (2007), 627-666. Zbl 1135.53046 MR 2336062

[39] S. Matveev, Algorithmic topology and classification of 3-manifolds. Algorithms Comput. Math. 9, Springer-Verlag, Berlin 2003. Zbl 1048.57001 MR 1997069

[40] W. Meeks and P. Scott, Finite group actions on 3-manifolds. Invent. Math. 86 (1986), 287-346. Zbl 0626.57006 MR 0856847

[41] J. Milnor, A unique decomposition theorem for 3-manifolds. Amer. J. Math. 84 (1962), 1-7. Zbl 0108.36501 MR 0142125

[42] J. Morgan and G. Tian, Completion of Perelman's proof of the geometrization conjecture. Preprint 2008. arXiv:0809.4040v1 [math.DG]

[43] G. Perelman, The entropy formula for the Ricci flow and its geometric applications. arXiv:arXiv:math/0211159v1 [math.DG]

[44] G. Perelman, Ricci flow with surgery on three-manifolds. arXiv:math/0303109v1 [math.DG] 
[45] G. Perelman, Finite extinction time for the solutions to the Ricci flow on certain threemanifolds. arXiv:math/0307245v1 [math.DG]

[46] A. Petrunin and W. Tuschmann, Diffeomorphism finiteness, positive pinching and second homotopy. Geom. Funct. Anal. 9 (1999), 736-774. Zbl 0941.53026 MR 1719602

[47] F. Prüfer, F. Tricerri, and L. Vanhecke, Curvature invariants, differential operators and local homogeneity. Trans. Amer. Math. Soc. 348 (1996), 4643-4652. Zbl 0867.53032 MR 1363946

[48] X. Rong, On the fundamental groups of manifolds with positive sectional curvature. Annals of Math. 143 (1996), 397-411. Zbl 0974.53029 MR 1381991

[49] X. Rong, Collapsed manifolds with bounded sectional curvature and applications. In Surveys in Differential Geometry XI, Metric and Comparison Geometry, ed. by J. Cheeger and K. Grove, International Press, Somerville, 1-23. Zbl 05342778 MR 2408262

[50] P. Scott, The geometries of 3-manifolds. Bull. London Math. Soc. 15 (1983), 401-487. Zbl 0561.57001 MR 0705527

[51] T. Shioya and T. Yamaguchi, Volume collapsed three-manifolds with a lower curvature bound. Math. Ann. 333 (2005), 131-155. Zbl 1087.53033 MR 2169831

[52] J. Song and G. Tian, The Kähler-Ricci flow on surfaces of positive Kodaira dimension. Invent. Math. 170 (2007), 609-653. Zbl 1134.53040 MR 2357504

[53] J. Streets, Ricci Yang-Mills flow. PhD thesis, Duke University 2007.

[54] R. Ye, Ricci flow, Einstein metrics and space forms. Trans. Amer. Math. Soc. 338 (1993), 871-896. Zbl 0804.53054 MR 1108615

Received May 19, 2008

John Lott, Department of Mathematics, University of California, Berkeley, CA 94720-3840, U.S.A.

E-mail: lott@math.berkeley.edu 